NBER WORKING PAPER SERIES

\title{
FIRM-LEVEL EXPOSURE TO EPIDEMIC DISEASES: \\ COVID-19, SARS, AND H1N1
}

Tarek Alexander Hassan

Stephan Hollander

Laurence van Lent

Markus Schwedeler

Ahmed Tahoun

Working Paper 26971

http://www.nber.org/papers/w26971

\author{
NATIONAL BUREAU OF ECONOMIC RESEARCH \\ 1050 Massachusetts Avenue \\ Cambridge, MA 02138
}

April 2020, Revised September 2022

We thank participants at the 2021 American Economic Association Annual Meeting, the EAA Virtual Accounting Research Seminar, 2021 European Economic Association Congress, NBER SI 2020 Asset Pricing, INQUIRE, Virtual Law \& Economics Workshop (University of Florida, University of Michigan, and University of Virginia), and Case Western Reserve. We thank Steve Davis, Ralph Koijen (discussant), Ken Kotz, and Tom Ferguson for helpful comments. Aakash Kalyani and Luke Melas-Kyriazi provided excellent research assistance. Tahoun sincerely appreciates support and funding from the Wheeler Institute for Business and Development. Tahoun and Van Lent sincerely appreciate the support from the Institute for New Economic Thinking (INET). Van Lent gratefully acknowledges funding from the Deutsche Forschungsgemeinschaft Project ID 403041268 - TRR 266. The dataset developed in this paper is available on www.firmlevelrisk.com. The views expressed herein are those of the authors and do not necessarily reflect the views of the National Bureau of Economic Research.

NBER working papers are circulated for discussion and comment purposes. They have not been peer-reviewed or been subject to the review by the NBER Board of Directors that accompanies official NBER publications.

(C) 2020 by Tarek Alexander Hassan, Stephan Hollander, Laurence van Lent, Markus Schwedeler, and Ahmed Tahoun. All rights reserved. Short sections of text, not to exceed two paragraphs, may be quoted without explicit permission provided that full credit, including $\odot$ notice, is given to the source. 
Firm-Level Exposure to Epidemic Diseases: COVID-19, SARS, and H1N1

Tarek Alexander Hassan, Stephan Hollander, Laurence van Lent, Markus Schwedeler, and Ahmed Tahoun

NBER Working Paper No. 26971

April 2020, Revised September 2022

JEL No. E0,E6,F0,G12,I0

\begin{abstract}
We construct text-based measures of the primary concerns listed firms associated with the spread of COVID-19 and other epidemic diseases. We identify which firms perceive to lose or gain from a given epidemic and textually decompose the epidemic's effect on the firm's demand and supply. We find that the effects of COVID-19 manifest as a simultaneous shock to demand and supply, with both shocks affecting firms' market valuations in equal measure on average. By contrast, demand-related impacts appear more important in accounting for the observed collapse in firmlevel investment during the COVID-19 crisis.
\end{abstract}

Tarek Alexander Hassan

Department of Economics

Boston University

270 Bay State Road

Boston, MA 02215

and NBER

thassan@bu.edu

Stephan Hollander

Tilburg University

Warandelaan 2

5037 AB Tilburg

the Netherlands

s.hollander@tilburguniversity.edu

Laurence van Lent

Frankfurt School of Finance and Management

Adickesallee 32-34

60322 Frankfurt am Main

Germany

1.vanlent@fs.de
Markus Schwedeler

Boston University

270 Bay State Road

Boston, MA 02215

mschwed@bu.edu

Ahmed Tahoun

London Business School

26 Sussex plc, Regent's Park

London NW1 4SA

United Kingdom

atahoun@london.edu

Data is available at www.firmlevelrisk.com 
"[D]o you want to touch on cancellations and just the whole hype around coronavirus?"

- Colin V. Reed, Chairman and CEO, Ryman Hospitality Properties Inc., in the company's Q4-2019 earnings call (February 25, 2020)

When the World Health Organization declared the outbreak of the COVID-19 virus a pandemic on March 11, 2020, the disease had already wreaked havoc in large swathes of China and Northern Italy. What started as a new illness in a middling city in China had grown within a few months to a global public health crisis unseen for a century. Stock markets around the world crashed. ${ }^{1}$ Even though governments rushed in equal measure to stem the further spread of the virus, locking down entire regions and supporting a suddenly wobbling economy, it became clear that the shock would leave few untouched.

While perhaps a singular event, the COVID-19 pandemic has underscored the need to quickly understand how firms are affected by and respond to large, aggregate, and unexpected events ("shocks"). However, those wishing to understand the ongoing or anticipated firmlevel impact of a large shock face four fundamental challenges related to measurement: How to identify which firm is affected? How to quantify the intensity of a firm's exposure to the shock? How to determine the nature of a firm's shock (for example, whether the shock results in a firm's demand contraction, supply disruption, or financial difficulties)? And finally, how to do so in time to still be useful for decision making?

A common approach to studying the firm-level impacts of large events is to examine earnings or related accounting data. However, accounting data are, by nature, backwardlooking and reflect news only at considerable lags. Further, when considering earnings in isolation, it would be hard to disentangle the impact of COVID-19 from that of other shocks and to identify - let alone quantify - the specific channels through which COVID-19 disrupts firms. That is, although one may observe firms' earnings being (negatively) impacted, it is much more difficult to determine whether, and if so by how much, this negative impact is due to COVID-19 impacting firms through their demand or supply side, or perhaps through

\footnotetext{
${ }^{1}$ See Baker et al. (2020); Gormsen and Koijen (2020); Ramelli and Wagner (2020); and Davis et al. (2021) for an early discussion of the stock market response to COVID-19.
} 
government regulations taken in response to the outbreak. ${ }^{2}$ Event studies (Kothari and Warner, 2007), which look at stock price reactions to events, may identify winners and losers but, likewise, fall short of giving clues about the channels through which a shock impacts a given firm.

At the same time, a detailed, firm-level understanding of a shock's distinct channels is essential for formulating an effective policy response. For example, while well-targeted monetary and fiscal policies can compensate for shortfalls in demand, they may fall flat or even cause inflation in the face of a contraction in supply. ${ }^{3}$ This is, of course, also true across countries, sectors, or even firms. Indeed, whereas firms from a specific country or sector may predominantly experience a demand shortfall, others could suffer from supplychain or financing difficulties. Thus, a better understanding of how a shock manifests at the firm level is essential for a more targeted response by monetary and fiscal authorities and for investors considering its consequences for their portfolios.

In this paper, we introduce a toolbox of text-based measures that allow researchers to systematically characterize the ongoing and anticipated impacts of large, potentially unprecedented events in near real-time. Specifically, these measures allow us to identify perceived winners and losers, trace the channels through which the event impacts specific firms, and identify firm-level demand and supply shocks resulting from the event. Since we use transcripts of earnings conference calls as our data source, the measures can be applied to virtually all US publicly listed firms and the majority of large internationally-listed firms in over 80 countries, and in near real-time (as soon as firms hold their earnings calls). We demonstrate the usefulness of the toolbox by analyzing which firms are affected by and how firms respond to the outbreak of COVID-19 and other epidemic diseases.

The keystone of our toolbox is the firm's exposure to the shock, where 'exposure' is

\footnotetext{
${ }^{2}$ Barrero et al. (2021) use data from a survey to underpin their conclusion that COVID-19 is a persistent reallocation shock, shifting employment growth to industries with a capacity for employees to work from home.

${ }^{3}$ See, for example, the debate in the literature about whether the Great Recession was demand-driven or due to a drop in productivity (e.g., Mian et al., 2013; Kaplan et al., 2020).
} 
defined as the proportion of the conversation in the earnings call devoted to the event of interest. ${ }^{4}$ This approach has been validated in recent work in the context of measuring a firm's exposure to political risk, Brexit, climate change, and other large shocks such as the Fukushima nuclear disaster (Hassan et al., 2019, 2020; Sautner et al., 2022). ${ }^{5}$ Building on the methods developed in that work, we further refine the exposure measure by constructing measures of the event's overall perceived impact on the mean (sentiment) and the variance (risk) of the firm's economic outlook. Doing so allows us to identify winners and losers, as the tone of the relevant discussion can be positive or negative, as well as the extent of risk and uncertainty that the firm associates with the shock. ${ }^{6}$

Second, we develop a new method that uses firms' exposure to the shock to systematically categorize the channels through which the shock impacts firms in near real-time. In our context, key questions on decision makers' minds were whether and to what extent the COVID-19 pandemic would contract supply vs. demand and whether the pandemic would precipitate a financial crisis. To speak to these issues, we used the first batch of earnings calls held in early 2020 to identify archetypal discussions of supply-side and demand-side impacts of the pandemic on each firm, as well as discussions of financial adjustments related to the pandemic. We then used a training set of manually-labeled discussions about these topics to develop a new, transparent, pattern-based classifier. The key benefit of this classifier is that it can be used to analyze the specific firm-level impacts of large events on thousands of listed firms around the world without the need for years of training data (likely not available

\footnotetext{
${ }^{4}$ In particular, as Hassan et al. (2019) describe, its usage is different from that common in the asset pricing literature where 'exposure' is mostly used to refer to a firm's sensitivity to an aggregate risk factor.

${ }^{5}$ Intuitively, the idea of constructing a measure of firm-level exposure to a particular shock from transcripts of periodic earnings calls rests on the observation that these conference calls are a venue in which senior management has to respond directly to questions from market participants regarding the firm's prospects. Not only are these disclosures therefore timely, seeing that earnings calls consist of a management presentation and, importantly, a Q\&A session, they also require management to comment on matters they might not otherwise have voluntarily proffered.

${ }^{6}$ In our context of discussions related to COVID-19, we find that sentiment more often describes ongoing impacts, whereas risk is predominantly associated with forward-looking language.
} 
at the onset of the shock) or for conducting costly and time-consuming surveys. ${ }^{7}$

Third, once we have identified which firms discuss the supply and demand impacts of the event, we can combine these data with the same sentiment libraries used in the first step of our analysis to obtain text-based measures of whether these impacts on supply and demand were good or bad for the firm. In other words, we construct explicit measures of exogenous supply and demand shocks at the firm level. These measures respect that, for example, some supply impacts may be worse than others and that demand impacts may be positive (e.g., supermarkets benefiting from restaurant closures) or - more commonlynegative (e.g., collapsing demand for air travel).

We then use this toolbox to study the micro-level consequences of COVID-19 in 2020 on firms in 82 different countries and compare them to those of prior epidemics. Our main findings are as follows. First, although perhaps less surprising from our current vantage point, we show how unprecedented in breadth and intensity the coronavirus pandemic was, even when compared with the most virulent epidemics in prior history. COVID-19 was a major topic of discussion for virtually all firms globally. In the second and third quarters of 2020, a remarkable three percent of sentences in earnings conference calls mention COVID19. While looking back, this pattern appears obvious; as the situation developed (i.e., at the time), however, this was much less so. Indeed, an important advantage of the text-based method we develop is that measurement is taken almost in real-time. Hence, we were able to make this assessment and circulate the first draft of this paper already in March of 2020.

Second, on average, firms report overwhelmingly negative impacts from COVID-19 on their businesses while also attributing a large increase in risks to the spread of the disease. In this sense, COVID-19 represents a shock to the mean and the variance of firms' fortunes. After a peak in pessimism associated with COVID-19 in June of 2020, the tone of discussion recovered in the third and fourth quarters of 2020, led by an uptick in optimism among

\footnotetext{
${ }^{7}$ Using a hold-out set of hand-labeled discussions, we evaluate the out-of-sample performance of our classifier and show that it outperforms three alternative classifiers trained on the same data: Naive Bayes, Logistic Regression, and Feedforward Neural Network.
} 
Asian firms. However, significant heterogeneity across firms and sectors exists underlying these overwhelmingly negative aggregate trends.

Third, moving beyond these important descriptive statistics, we examine the valuation effects of the COVID-19 shock. Using quarterly stock returns in 2020, we find negative valuation effects for firms with more exposure to COVID-19. Decomposing COVID-19 exposure into risk and sentiment components suggests that negative sentiment related to the COVID19 outbreak is the prevalent factor explaining returns. Less expected, perhaps, measured COVID-19 risk also negatively affects returns and correlates strongly with increased stock market volatility.

Fourth, taking advantage of our text-based method to probe deeper into the specific concerns firms associate with COVID-19, we find that the pandemic manifests itself at the firm level as a simultaneous supply and demand shock, with supply concerns significantly larger during the COVID-19 crisis than in any earlier epidemic. Indeed, during the COVID19 crisis, firms appeared concerned about the supply and demand-related impacts of the pandemic in almost equal measure, whereas prior epidemics triggered demand-related concerns predominantly. Even at the onset of the coronavirus pandemic, many firms highlighted concerns about their supply chains. Whereas policymakers sometimes struggled to understand how they could support the economy, even in these early stages of the pandemic, the (earnings-call-based) data thus provided strong clues about potential bottlenecks. In regions, for example, where the COVID-19 outbreak is more virulent, supply impacts tend to be more significant (perhaps due to stricter lockdown measures or other public health restrictions).

Finally, when we isolate from these concerns the extent to which a firm is exposed to exogenous COVID-19 demand and supply shocks, we find that the supply and demand impacts of COVID-19 are both important in explaining variation in stock returns in 2020. For example, a one-standard-deviation increase in the pandemic's negative supply impacts on a given firm is associated with a 1.0 percentage point lower stock market valuation in the 
cross-section. Similarly, contractions in both supply and demand appear to have driven a drop in employment among large listed firms in the United States, which were not eligible for government subsidies for maintaining jobs. By contrast, negative demand impacts appear to be the driving force behind the significant decline in firm-level investment of all firms: a one-standard-deviation increase in the pandemic's negative demand shock is associated with a 3.7 percent decrease in firms' investment.

Stepping back, we hope that a deeper understanding of how large shocks affect firms may facilitate the development of effective government and/or corporate intervention policies. More fundamentally, however, our methodological innovation-i.e., introducing a wordbased pattern approach to determining whether a shock-related text fragment discusses a given channel-has broader applications and can be readily adapted for a range of tasks involving algorithmic classification of text in earnings-call transcripts and other disclosures. Our approach economizes on the amount of training data needed for the text classification algorithm to learn about the channels through which the shock operates at the firm level, making it a useful tool for the (typically) data-scarce conditions when attempting to analyze new shocks in real time.

Related literature. Distinguishing empirically between supply and demand impacts of shocks has long been an open question in macroeconomics (e.g., Blanchard and Quah, 1989). Doing so is crucial not only for formulating an effective policy response in response to crises (which is our focus here), but also for testing basic tenants of the New Keynesian model (e.g., Nekarda and Ramey, 2020), and studying the propagation of shocks in production networks (e.g., Long and Plosser, 1983; Acemoglu et al., 2017; Baqaee and Farhi, 2019). A number of recent papers have made progress by measuring the transmission of supply shocks across firms following natural disasters (e.g., Barrot and Sauvagnat, 2016; Boehm et al., 2019; Carvalho et al., 2021). However, these methods require exceptionally detailed data on customer-supplier networks, accounting data that are available only at considerable lags, and are generally confined to studying the propagation of a specific type of supply 
shock. We contribute to this literature by introducing a flexible method for measuring the differential exposure of firms to a large shock which is available in near real-time and can pinpoint whether a given firm is concerned with supply impacts, demand impacts, or other concerns. Thus, we learn about the channel by which a firm is impacted by the shock, as perceived by corporate managers and other participants in the earnings call.

We illustrate our method using the setting of the coronavirus pandemic. Consequently, the paper also contributes to the fast-growing literature on the economics and finance of COVID-19. ${ }^{8}$ The discussion in these studies centers around understanding whether the economic consequences of COVID-19 are best understood as the pandemic causing a demand shock or a supply shock (Guerrieri et al., 2022; Baqaee and Farhi, 2020; Bekaert et al., 2020; Fornaro and Wolf, 2020; Faria-e Castro, 2021). Depending on the answer to this question, the optimal policy response of governments varies. Most immediately, conventional wisdom holds that monetary and fiscal stimuli may be effective remedies in countering negative demand shocks but are ineffective (and even inflationary) in the face of a supply shock. Understanding whether, and to what extent COVID-19 represents the former or the latter is thus crucial for formulating effective policy. ${ }^{9}$

In finance, several studies highlight the credit market access and liquidity consequences of the COVID-19 pandemic (Au et al., 2020; Ferrando, 2020; Kargar et al., 2021; Ma et al., 2020; Ozik et al., 2021; Greenwald et al., 2021). Several papers study the impact on the cross-section of equity returns (Alfaro et al., 2020; Bretscher et al., 2020). Stock prices, on average, plunged initially but rebounded in the second half of 2020. This general pattern, however, masks important heterogeneity across firms. Alternative proposals to capture this

\footnotetext{
${ }^{8}$ In addition, there is a large literature in development and health economics studying pandemics, either in general or specific diseases (e.g. Fogli and Veldkamp, 2021; Greenwood et al., 2019; Philipson, 1999).

${ }^{9}$ Atkeson (2020) and Eichenbaum et al. (2021) argue for integrating epidemic models of the spread of a disease with conventional macroeconomic models to study the effect of policy interventions in this context. Ding et al. (2021) use data on COVID-19 cases to measure changes in the economy's exposure to the pandemic. Important other studies investigating the policy response (and its economic impact) to the COVID-19 pandemic include work that examines social distancing rules (Barro et al., 2020), lockdowns (Alon et al., 2020; Arnon et al., 2020; Kaplan et al., 2020; Moser and Yared, 2021), and the Paycheck Protection Program (Joaquim and Netto, 2020).
} 
heterogeneity are in Davis et al. (2021) and Stephany et al. (2022), who rely on regulatory filings to characterize differential reactions to COVID-19 news, and Croce et al. (2020), who use high-frequency data from Twitter to measure epidemic contagion risk in financial markets. ${ }^{10,11}$

\section{DATA}

We use transcripts of quarterly earnings conference calls held by publicly-listed firms to construct our measures of firm-level exposure to epidemic diseases. These transcripts are available from Refinitiv Eikon. We collect the complete set of 333,626 English-language transcripts from January 1, 2002, to December 31, 2020, for 12,952 firms headquartered in 82 countries. $^{12}$ Earnings calls are key corporate events on the investor relations calendar and allow financial analysts and other market participants to listen to senior management presenting their views on the company's state of affairs and ask these company officials questions about the firm's financial performance over the past quarter and, more broadly, discuss current developments (Hollander et al., 2010). Appendix Table 1 presents the details of the extensive global coverage of listed firms in our sample.

We also use financial statement data from Standard \& Poor's Compustat North America

\footnotetext{
${ }^{10}$ Stephany et al. (2022) use the text in $10-\mathrm{K}$ filings to track the evolution of eight COVID-19 related topics at the industry and aggregate levels. They, however, do not broach the identification of exogenous shocks nor their firm-level impacts. Davis et al. (2021) use discussions of risk factors (i.e., 'Item 1A') in pre-pandemic 10-K filings to explain firm-level returns on 20 "jump" days from February 24 to March 27, 2020, when the market rose or fell by at least 2.5 percent. Thus, they seek to inform an understanding of which of a long list of text-based risk categories (e.g., 'inflation,' 'litigation matters,' 'monetary policy') drives firm-level return reactions to the COVID-19 shock. By contrast, our paper uses NLP measures directly from the corona-related concerns managers and non-corporate participants articulate in quarterly earnings calls, for a large panel of listed firms around the globe (i.e., not just US-listed firms). Thus, while close in spirit, both papers differ markedly in the data source, method, purpose, and research questions from ours.

${ }^{11}$ More broadly, we also add to the growing literature in finance and related fields using text as data (Gentzkow et al., 2019). A number of other recent papers use firm-specific text documents to measure firm-level political risk (Hassan et al., 2019), overall risk (Handley and Li, 2018), climate change exposure (Sautner et al., 2022), cyber risk (Jamilov et al., 2021), and trade policy risk (Caldara et al., 2019; Kost, 2019). Similarly, others have used newspaper articles and FOMC minutes to measure economic policy uncertainty (Baker et al., 2016), the state of the economy (Bybee et al., 2019), and analyze news about monetary policy (Hansen et al., 2018).

${ }^{12}$ This description applies at the moment of writing this paper. The publicly available dataset on www. firmlevelrisk.com is continuously updated as new transcripts become available.
} 
and Global, including data on firms' total assets, revenue, and investment rate; we use the location of firms' headquarters from Refinitiv Eikon. ${ }^{13}$ We convert all non-USD-denominated variables from financial statements into USD. Stock return data are from Refinitiv Eikon. Table 1 provides summary statistics.

\section{Epidemic Disease Exposure, Risk, And Sentiment}

\subsection{Measurement}

Our first aim is to construct a firm-level measure of exposure to COVID-19 and other epidemic diseases. We use the approach developed in Hassan et al. (2019, 2020) to identify whether a given disease outbreak is discussed in a quarterly earnings call. We then use the extent of this discussion to measure firm-level exposure to this disease and analyze the surrounding text to assess the sentiment and risks the firm associates with the outbreak.

We begin by determining the epidemic disease outbreaks that occurred within our sample period (which starts in 2002) from the list of pandemic and epidemic diseases maintained by the World Health Organization (WHO). ${ }^{14}$ We restrict the list to diseases that, in our judgment, attracted a sufficient international audience and potentially were a concern to equity investors. This restriction eliminates outbreaks such as the 2019 Chikungunya episode in Congo and the 2018 Monkeypox in Nigeria.

For the remaining list of outbreaks, we identify the most common synonyms for each disease in online resources and newspaper articles at the time of the event. We also read a limited sample of earnings-call transcripts to verify the disease words (combinations) used during each of these outbreaks. Finally, we ensure that disease words (combinations) have no alternate meaning, such as is the case for MERS and the "Malaysian Emergency Response Services 999." Appendix Table 2 lists the words (combinations) used per disease.

Having thus compiled our disease word list, our time-varying measure of a given firm's

\footnotetext{
${ }^{13}$ Note that this latter variable is meant to measure the location of a firm's operational headquarters rather than the country of incorporation, which is often distorted by tax avoidance strategies.

14 www.who.int/emergencies/diseases/en/.
} 
exposure to epidemic disease $d$, denoted DiseaseExposure ${ }^{d}$, is constructed by counting the number of times the synonyms associated with each disease $d$ are used. We then divide this number by the total number of words in the transcript to account for differences in transcript length:

$$
\text { DiseaseExposure }{ }_{i t}^{d}=\frac{1}{B_{i t}} \sum_{b=1}^{B_{i t}} 1\left[b \in \mathcal{D}_{d}\right] \text {, }
$$

where $b=0,1, \ldots, B_{i t}$ index the words contained in the transcript of firm $i$ in quarter $t, B_{i t}$ is the total number of words in the transcript, and $\mathcal{D}_{d}$ is the set of disease words or word combinations associated with disease $d$.

Based on this epidemic disease exposure measure, we also construct metrics of risk and sentiment, denoted DiseaseRisk ${ }_{i, t}^{d}$ and DiseaseSentiment $t_{i, t}^{d}$, respectively (Hassan et al., 2019, 2020). Specifically, DiseaseRisk $k_{i, t}^{d}$ conditions the count in (1) on proximity to synonyms for risk or uncertainty: it counts only the words (word combinations) related to a specific disease that occur in the neighborhood of 10 words before and after such a synonym. ${ }^{15}$ We interpret DiseaseRisk ${ }_{i, t}^{d}$ as a measure of the risk firm $i$ associates with the spread of disease $d$ at time $t$.

To gauge whether a disease outbreak is deemed good or bad news for a given firm, we also construct a measure of how the spread of a given disease affects the firm's prospects. ${ }^{16}$ Accordingly, the construction of epidemic disease sentiment, denoted DiseaseSentiment ${ }^{d}$, closely follows the procedure for DiseaseRisk ${ }^{d}$ by counting the words associated with disease $d$; however, instead of conditioning on the proximity to terms related to risk (i.e., the second moment), this time we count positive minus negative-tone words surrounding the mention of the disease to capture the first moment. These positive- and negative-tone expressions are

\footnotetext{
${ }^{15}$ We obtain a list of synonyms for "risk" and "uncertainty" from the Oxford English Dictionary. See Appendix Table 3 for a list of these synonyms.

${ }^{16}$ Having such a measure is also helpful to address the issue that innovations to the variance of shocks (risk) are likely correlated with innovations to the conditional mean. Thus, teasing out the effects of diseaserelated uncertainty on a firm's actions also requires controlling for the impact of the disease event on the conditional mean of the firm's future earnings.
} 
obtained from Loughran and McDonald (2011). ${ }^{17}$ (Positive words include 'good,' 'strong,' and 'great,' while negative words include 'loss,' 'decline,' and 'difficult. ${ }^{18,19}$ Appendix Table 4 shows our corpus' most frequently used tone words.) As expected, descriptive statistics suggest that negative-tone words dominate disease-related discussions in earnings-call transcripts. Accordingly, in subsequent analysis, we sometimes bifurcate DiseaseSentiment ${ }^{d}$ into DiseaseNegativeSentiment ${ }^{d}$ and DiseasePositiveSentiment ${ }^{d}$, simply by conditioning on either negative or positive sentiment words, respectively.

\subsection{Descriptive Findings}

In this and the next subsection, we use our newly developed measures of firm-level disease exposure, risk, and sentiment to document several stylized facts. Then, in Sections 3 and 4, we will unpack the specific concerns voiced in a firm's earnings call about how a given outbreak affects the firm. We emphasize firm-level exposure to the 2020 coronavirus pandemic, but we also present some findings on the earlier epidemic diseases in our sample period.

Indeed, Figure 1 depicts the time series of the percentage of transcripts in which a given disease is mentioned in a quarter separately for COVID-19, SARS, H1N1, Ebola, Zika, and MERS, respectively (moving from the top panel to the bottom). ${ }^{20}$ Reassuringly, these patterns closely follow the infection rates for each of the diseases in the population. For example, according to the WHO, SARS was first recognized in February 2003 (although the outbreak was later traced back to November 2002), and the epidemic ended in July 2003. Accordingly, discussions of SARS in earnings conference calls peak in the first quarter of 2003 and quickly trail off after the epidemic ends. Finally, SARS, a disease likewise caused

\footnotetext{
${ }^{17}$ Thirteen of the synonyms for risk or uncertainty used in our sample earnings-call transcripts also have negative tone according to this definition. Examples include 'exposed,' 'threat,' 'doubt,' and 'fear.' Our measures thus explicitly allow speakers to convey risk and negative sentiment simultaneously.

${ }^{18}$ We choose to sum across positive and negative sentiment words rather than simply conditioning on their presence to allow multiple positive words to outweigh the use of one negative word, and vice versa.

${ }^{19}$ One potential concern that has been raised with this kind of sentiment analysis is the use of negation, such as 'not good,' or 'not terrible' (Loughran and McDonald, 2016). However, we have found that such negation is not common in our sample, so we chose not to complicate the construction of our measures by explicitly allowing for it.

${ }^{20}$ Our sample currently ends with earnings calls held through December 31, 2020.
} 
by a coronavirus, returns as a subject in earnings calls in the first quarter of 2020, when it becomes clear that COVID-19 shares some commonalities with the former outbreak.

The figure highlights once more how exceptional COVID-19 is. Indeed, forty percent of transcripts discuss the outbreak in the first quarter of 2020 and almost 100 percent after that. This proportion is much larger than in previous episodes (with SARS as the closest "competitor" at just over 20 percent). In Appendix Figure 1, we provide additional detail for the separate cases of China, the United States, and Europe (including the UK). Interestingly, SARS was a pervasive topic of discussion in China (at levels similar to COVID-19). In contrast, the Ebola virus did not feature in earnings calls of firms headquartered in China.

In Figure 2, we zoom in on the first few months in which a given disease occurs and compare by region in which a firm is headquartered the weekly average corporate exposure to COVID-19, SARS, and H1N1, respectively (moving from the top panel to the bottom). One immediate takeaway from comparing the plots is that COVID-19 prevails in discussions in earnings calls. During Q3 of 2020, more than three percent of all sentences in our sample transcripts contain a discussion of COVID-19. (For comparison, only 0.7 percent of sentences in the average transcript in our sample mention 'competition,' 'competitive,' 'compete,' 'competing,' or 'competitor.') Further, during their epidemic episode, the exposure to diseases is much less synchronized for SARS and H1N1 than for COVID-19, which is rising simultaneously in all parts of the world. The saw-tooth patterns in the cases of SARS and H1N1 signify that earnings-call discussions of the disease peaked sequentially in different regions around the world during these outbreaks, with early peaks representing regions in which the disease was first discovered. In contrast, COVID-19 exposure grows rapidly between April and May 2020 in all areas except China and remains high. For companies headquartered in China, much of the acceleration in exposure occurs before April, consistent with the outbreak affecting the country hard in the first few months. Firm-level exposure to SARS and H1N1, again consistent with the development of infection rates in the population, climbs first in Asia and Mexico, respectively: the putative origin regions of the two diseases. 


\subsection{Winners, Losers, and Risk in the COVID-19 Pandemic}

To assess the firm-level impact of exposure to COVID-19 in the opening months of 2020, we plot the weekly average COVID-19 risk and sentiment scores in Figure 3. We observe relatively low COVID-19 risk and slightly negative sentiment in January and February, but by March, the weekly average COVID-19 risk climbs quickly and reaches a maximum in early May. These developments are mirrored in the weekly average sentiment during the same period, which declines precipitously from March to early July. From June onward, COVID-19 risk remains high (although never reaching the levels of May again) until the end of the sample period. In contrast, COVID-19 sentiment improves markedly during Q3 of 2020, albeit that sentiment remains negative overall. ${ }^{21}$ For the average firm, COVID-19 is not only bad news but exposes management to significantly higher uncertainty. ${ }^{22}$

To interpret these statistics better, we conduct a human audit where we examine the text fragments that underlie our COVID-19 risk and sentiment scores to assess whether call participants were discussing the impact of ongoing or past events or, on the other hand, whether the discussions are forward-looking in nature. We find that $81 \%$ of text fragments underlying our COVID-19 sentiment measure discuss impacts that are ongoing or occurred in the recent past, with the remaining $19 \%$ focusing on anticipated future impacts. By contrast, $76 \%$ of text fragments underlying our COVID-19 risk measure focus on possible future impacts. (Appendix Table 6 provides further details.) In this sense, COVID-19 sentiment ${ }_{i, t}$ may be best thought of as tracking the ongoing impact of the pandemic, whereas COVID-19 risk, truly captures uncertainty about anticipated future impacts.

These aggregate patterns are important and interesting in their own right but mask

\footnotetext{
${ }^{21}$ In Appendix Figure 2, we document that the improvement in sentiment after the first quarter is driven mainly by a more positive outlook among Asian firms.

${ }^{22}$ Intuitively, the extent to which a population is exposed to a disease in a region should be associated with firms' exposure to the same. Thus, infection rates should be correlated with our firm-level exposure measures. We explore this relation in Appendix Table 5. In short, we find that infection and mortality rates in a country are positively associated with COVID-19 negative sentiment ${ }_{i, t}$, implying that more infections go hand in hand with negatively toned discussions about the coronavirus in the earnings calls. As expected, COVID-19 exposure $_{i, t}$ is also positively associated with infection rates.
} 
considerable variation at the sector level, as shown in Panel A of Figure 4. High COVID19 risk is found in sectors such as basic materials, healthcare, and technology, whereas the perceived risk associated with COVID-19 is noticeably lower for the energy and utilities sector. Importantly, the average sentiment is negative across all sectors. Still, at the same time, outlooks are much less negative in the technology, healthcare, and consumer noncyclical sectors than in the transportation and energy and utilities sectors. These patterns make intuitive sense. Indeed, while the crisis severely decreased travel and oil demand, some supermarkets and tech firms saw their businesses expand as people increasingly worked and dined at home. At the same time, the healthcare sector faced tremendous changes and volatility as COVID-19 questions the ability to deliver these services in person (high risk).

\subsection{Valuation Effects of COVID-19 Exposure, Sentiment, and Risk}

We next ask whether differential COVID-19 exposure, sentiment, and/or risk can account for variation in stock price changes as measured by (1) the annualized quarterly stock return in each of the four quarters of 2020 or (2) over a short (three-day) window centered on the earnings call date (using earnings calls held in all four quarters of 2020). Intuitively, standard asset pricing models suggest that a change in stock price occurs when investors, in aggregate, revise their views on expected future cash flows and/or the expected discount rate. Thus, more positive sentiment about an epidemic disease should be associated with increased returns, whereas a higher perceived risk should be negatively associated with the same.

We test these predictions using the following regression:

$$
\operatorname{Ret}_{i, t}=\alpha_{0}+\delta_{t}+\delta_{s}+\beta C O V I D-19 X_{i, t}+Z_{i}^{\prime} \nu+\epsilon_{i, t},
$$

where $\operatorname{Ret}_{i, t}$ is either the annualized quarterly return or the cumulative return over a threeday $(-1,1)$ window around the date of the earnings call; COVID-19 $X_{i, t}$ is either our COVID- 
19 exposure, sentiment, or risk score. We also split COVID-19 sentiment ${ }_{i, t}$ into a negative and positive sentiment variable, to document the association between positive (negative) COVID-19 news and returns. The vector $Z_{i}$ includes our standard set of control variables; specifically, the natural logarithm of firm $i$ 's total assets (as a control for the size of the firm) and its market beta, calculated by regressing firm $i$ 's daily returns in 2018 on the return of the S\&P 500 index (to measure the firm's exposure to the US capital market). ${ }^{23}$ We include both quarter $\left(\delta_{t}\right)$ and two-digit SIC sector $\left(\delta_{s}\right)$ fixed effects. In all regressions, standard errors are clustered at the firm level.

Table 2, Panel A presents our estimation results at the firm-quarter level using annualized quarterly returns over the four quarters of 2020 as the dependent variable, which we detail for the full sample (columns 1-3) and separately for the US (columns 4-6). We see a significantly negative association between a firm's COVID-19 exposure and its stock return (in columns 1 and 3). Thus, firms with more extensive discussions in their earnings calls about the COVID19 outbreak experience a greater stock price decline on average than firms with less exposure. For example, in column 1, a one-standard-deviation increase in COVID-19 exposure $_{i, t}$ is associated with an 8.9 percentage point lower annualized return in the quarter of the earnings call. The equivalent for the US-based sample (in column 4) is 12.0 percentage points.

Next, we consider whether this return response derives from investors revising their expectations of cash flows, as measured by COVID-19 sentiment $t_{i, t}$, or their perceptions of the firm's risk, as captured by COVID-19 risk , $_{i, t}$ When regressing these variables onto returns, results show that both explain variation (columns 2 and 5). Note, however, that when we separate the positive and negative sentiment in columns 3 and 6 , only the association between COVID-19 negative sentiment $t_{i, t}$ and returns remains consistently negative and significant for both the full and US samples (the magnitude of the coefficients tends to remain stable across specifications). For example, in column 3, a one-standard-deviation increase in negative COVID-19 sentiment is associated with a 5.9 percentage point decrease in stock

\footnotetext{
${ }^{23}$ Summary statistics for all variables are reported in Table 1. For ease of interpretation, we standardize all firm-level exposure, sentiment, and risk variables by their standard deviation in the panel.
} 
returns. ${ }^{24}$

In Panel B, we examine the short-window returns surrounding the earnings call date in which COVID-19 is discussed. We again see a significant negative association between COVID-19 exposure $_{i, t}$ and three-day earnings-call returns (columns 1 and 4), consistent with the view that earnings conference calls reveal some incremental information about firms' COVID-19 exposure. In column 1, the estimated coefficient implies that a one-standarddeviation increase in COVID-19 exposure $_{i, t}$ is associated with a 0.3 percentage point lower return in this narrow window around the conference call. We also find that the short-window returns are significantly associated with COVID-19 sentiment sit, $_{\text {but not with COVID-19 risk }}$ (columns 2 and 5), though even the latter retains the predicted sign.

Expanding on this theme, Panel A of Appendix Table 7 probes the relation between our measures of interest (COVID-19 exposure, risk, and sentiment) and realized volatility. As expected, it shows that firms with higher COVID-19 exposure exhibit significantly higher volatility, while those with more positive COVID-19 sentiment exhibit lower volatility. Importantly, COVID-19 risk is positively associated with realized volatility even when we simultaneously control for COVID-19 sentiment. ${ }^{25}$

We conclude that our measures of COVID-19 risk and sentiment contain information relevant to firms' fortunes during the coronavirus pandemic (Panel A of Table 2) and that some of this information may originally have been transmitted to markets through earnings conference calls (Panel B of Table 2). Moreover, the fall and subsequent recovery in aggregate stock prices in Q1 and Q3 of 2020 mask significant COVID-19-induced heterogeneity in the cross-section of firms. We aim to systematically exploit the discussions of how firms are affected by the pandemic in the next section, in which we identify specific COVID-19-related impacts, responses, and concerns, as voiced in the earnings-call transcripts, and use this to

\footnotetext{
${ }^{24}$ See, for example, Giglio et al. (2021) for a study showing how stock returns changed during the FebruaryMarch 2020 stock market crash induced by the COVID-19 pandemic.

${ }^{25}$ Also in line with expectations, Panel B of Appendix Table 7 shows that more negative supply and demand impacts (which we describe and measure in the following section) are associated with higher volatility, with and without controlling for COVID-19 risk.
} 
shed light on how the valuation effects documented in this section are related to the type of shocks to which firms are exposed.

\section{Channels: Firm-level Impacts, Responses, and Concerns}

\subsection{Using Pattern-Matching to Identify Channels}

Having the full conference call conversation available in the form of a transcript allows us to probe deeper into the underlying concerns of management and call participants to understand how (i.e., through which channel(s)) an epidemic disease impacts corporate policies and performance. However, doing so systematically for all firms in our sample poses a challenge because of the sheer volume of text fragments that need to be processed and classified to identify the issues discussed by participants on a call. Indeed, focusing only on the 2020 coronavirus outbreak, 14,765 earnings-call transcripts mention a COVID-19 keyword. When we single out all text fragments within a given transcript that include COVID-19 keywords, we find 174,582 sentence triples: i.e., a set of three consecutive sentences by the same speaker with the middle sentence containing a COVID-19-related keyword. ${ }^{26}$ Therefore, rather than relying on a human reading of these text fragments, we develop a word patternbased algorithm below. The key feature of this algorithm is that it injects expert human judgment at a critical stage, ensuring that the classifier is transparent, relies on clear and intuitive rules, and can be applied at scale, even when no large training dataset is availableas is the case when analyzing a new, unprecedented shock, such as the COVID-19 pandemic. We describe the procedure step-by-step below.

Step 1: Selecting channel categories. We start by determining the mixture of topics that firms discuss when mentioning a COVID-19 keyword. To this end, the author team read a large number of randomly selected COVID-19-related sentence triples to define broad categories that are economically meaningful and capture as many of the coronavirus-related

\footnotetext{
${ }^{26}$ We use this sentence triple as the unit of analysis for our topic classification because doing so provides more context to infer the topic associated with mentioning the COVID-19 keyword.
} 
discussions as possible. Further, the categories should also be sharply delineated to minimize classification ambiguity in our automated reading of the sentence triples that follows next. We identify the following five key channel categories: (1) demand impacts, (2) supply impacts (which include discussions related to (a) supply chain and (b) production and operations), as well as three sets of actions taken to mitigate and manage these impacts: (3) cost reductions, (4) financial adjustments, and (5) government assistance. ${ }^{27}$

In keeping with our goal to isolate firm-level supply and demand shocks, as described below, channels (1) and (2) allow only discussions of impacts of COVID-19 on supply and demand that are exogenous to the firm, such as, for example, statements like "The decline in net revenue was primarily due to the impact of COVID-19," or "we've been facing countrywide lockdowns ... resulting in significant reductions in production capacity." By contrast, we allow the remaining three channels to also include discussions of indirect impacts and endogenous responses such as the effect of the crisis on firms' profits and cash flows, as well as measures taken by management to preserve liquidity and reduce costs. We also allow for a residual category that collects all other disease references; including, in particular, those unspecific as to the actual impact on the firm (e.g., "There is no doubt that COVID-19 is impacting our business").

Step 2: Developing channel-specific word patterns. Having determined these five key channel categories and a residual, our next task is to automatically classify all sentence

\footnotetext{
${ }^{27}$ As shown in Appendix Figure 3, and further discussed in Appendix A, we also employ a commonly used unsupervised approach, Latent Dirichlet Allocation (LDA), for discovering topics. The word clouds in this figure suggest that none aligns with what economists view as supply or demand-related impacts. We believe this finding is not accidental but instead results from a key logical issue that limits the applicability of automated topic detection in this context: conversations in earnings calls are multi-dimensional by nature, and 'off-the-shelf' LDA algorithms have no way of telling which of these dimensions are economically relevant. For example, a typical discussion of a supply-side impact might read: "On the top line, organic sales in the first quarter declined by $1.3 \%$, including the negative impact of our facilities in China being closed for a full month due to the COVID-19 pandemic." This discussion touches on topics on multiple logical planes: the firm's total profits, closed facilities, supply-side impacts, and the firm's activities in China. LDA attempts to cluster topics discussed and, instead of identifying the relation between this firm's closed facilities and another firm's difficulties in sourcing parts, might find that this discussion is closest to other discussions of the firms' foreign activities, profits, or closed facilities. All of these inferences are, of course, correct. Deciding on which of these dimensions we are interested in is thus inherently a task that requires judgment, which we exert by defining topics ourselves.
} 
triples into these categories. It is worth noting that this can be a difficult task even for the human reader, let alone for a computer algorithm, because how conference call participants discuss each channel tends to vary considerably. For example, there are subtle variations in how corporate managers may discuss disruptions of their supply chains: rather than mentioning supply chains explicitly, they might say that a health crisis impacts their ability to source components. Therefore, the challenge of this second step is to do justice to such subtle variations. ${ }^{28}$

To meet this challenge, we develop an iterative procedure that combines limited human judgment with data-driven decisions to identify a word pattern for each of our five specific channels. When applied to a text, such a word pattern should be able to reliably identify whether the text is about the channel that the word pattern aims to identify and transparently justify its classification by providing the words that lead to the match. We define it to consist of two components: (1) a set of phrases (contiguous groupings of words) that are directly related to a given channel, and (2) a set of (possibly non-contiguous) word combinations that, when used together within a sentence triple, indicate the channel is being discussed.

To obtain such word patterns for each of the five channels, we read and hand-label 600 randomly selected sentence triples that mention COVID-19 from our earnings-call transcripts, 437 of which we can unambiguously assign to at least one of our five channels. ${ }^{29}$ This collection is our training dataset. We then iteratively devise a word pattern for each channel to balance correctly predicting the labels of these hand-labeled sentence triples with accurately predicting the content of previously unseen sentence triples (validation dataset). Balancing the predictive performance on these two datasets helps prevent overfitting on the

\footnotetext{
${ }^{28}$ With a sufficiently large labeled training dataset, one could train a neural network, which tends to perform well with supervised classification tasks. However, this would require hand-labeling thousands of sentence triples. As we are interested in developing a method for measuring the impact of an evolving shock in real-time (i.e., as it unfolds), training data can be scarce. For sake of comparison, we train a neural network (discussed in further detail below), albeit with a small training dataset.

${ }^{29} \mathrm{As}$ we were conducting this analysis in real-time, all of these 600 snippets were sampled from earnings calls held in the first two quarters of 2020.
} 
training dataset.

More specifically, we start by defining the word pattern as a small set of phrases that frequently occur in a given topic's training dataset and are economically closely linked to the topic (e.g., 'supplier' for the 'supply impacts' channel category). We then check the fit of the pattern in our training dataset. By examining false positives and false negatives, we update the pattern (e.g., expand the set of phrases) to improve the in-sample fit. ${ }^{30}$ We continue this process until the pattern predicts the labels in our training dataset with no more than ten false positives and false negatives. Once this threshold is met, we audit the pattern with a validation dataset created by randomly drawing 30 sentence triples from the population of sentence triples matching the pattern out-of-sample. We then read these text excerpts and classify them as true or false positive matches to the predicted channel category. We stop and save the pattern if this audit produces fewer than eight false positives. If not, we adjust the pattern so that its predictive performance on the validation set meets the threshold before reexamining the updated pattern's performance on our training dataset and, if needed, iterating and auditing again with another validation dataset. Once we have arrived at a pattern that meets both criteria, the iteration ceases. ${ }^{31}$

Table 3 shows our final word patterns for each of the five channels, separating the 'supply chain' and 'production and operations' subcategories within 'supply impacts.' (These two aspects of supply disruptions arose naturally in our classification and seemed sufficiently distinct to warrant separate measurement.) To make the table easier to read, we abstract

\footnotetext{
${ }^{30}$ To expedite this process of improving in-sample fit, we found it useful to use embedding vectors trained on earnings conference calls and lexical databases to identify closely-related words that often co-occur with words in the pattern.

${ }^{31}$ Appendix Figure 4 uses "confusion matrices" to report our algorithm's fit to the training dataset for each of our topic categories (again separating the "supply chain" and "production and operations" sub-categories within the "supply impacts" topic category). Each matrix shows, for each individual topic category, the number of true positives, false positives, true negatives, and false negatives. For example, Panel A shows that the algorithm correctly labels 134 sentence triples related to "demand impacts," producing six false positives and seven false negatives. Two hundred ninety sentence triples relating to one of the other topics are correctly identified as not relating to "demand impacts." Appendix Table 8 shows the results of the last (out-of-sample) validation test performed in our iterative process. All but one topic category are near or below five false positives; the highest number of false positives is eight for the sub-topic category "production and operations."
} 
from stemming, although our algorithm allows for it (so that, for example, the word 'challenge' also allows for 'challenges' and 'challenging'), and all nouns apply both in singular and plural. In addition to the words and phrases listed in the table, each channel comes with a list of exclusions (reported in detail in Appendix Table 9). Table 3 confirms that the word patterns are very intuitive. For example, the 'production and operations' subcategory features discussions of (government) permits, productivity, throughput, closures, and shutdowns in conjunction with a mention of an epidemic disease.

Step 3: Classifying sentence triples. We then classify all 174,582 sentence triples (minus the hand-labeled training data) in our sample that mention COVID-19 and define

$$
\text { COVID-19 channel }{ }_{i t}^{C}=\frac{1}{S_{i t}} \sum_{s=1}^{S_{i t}}\left\{1\left[s \in \mathbb{P}^{C}\right]\right\}
$$

where $S_{i t}$ is now the total number of sentence triples in the transcript of firm $i$ in quarter $t$, and $\mathbb{P}^{C}$ is the set of patterns associated with one of the five channel categories $C$.

\subsection{Empirical Findings}

Figure 5 presents the findings from applying our pattern-based algorithm to the full sample of coronavirus sentence triples. For each month in 2020, the figure depicts the proportion of COVID-19-centered discussion devoted to each of our five channels: i.e., demand impacts, supply impacts, cost reductions, financial adjustments, and government assistance. (Appendix Table 10 gives examples for each channel.) As shown, the sudden change in demand is the most commonly voiced concern when the discussion turns to the pandemic. Indeed, 41.53 percent of all sentence triples mention demand impacts, followed by $32.39 \%$ of snippets discussing supply impacts. Among the latter, supply chain-related issues make up 5.69 percent of mentions on average, while impacts relating to production and operations account for the remainder (26.70 percent). Supply chain concerns peaked in the first two months of 2020 and decreased quickly thereafter. At the same time, discussions about production and 
operations (including forced closures of sites and stores) increased from March and remained at about the same level after Q3.

Higher costs and the imposition of cost-saving measures due to COVID-19 represent a further concern. These discussions represent mostly managerial responses to the pandemic shock in as much as managers observe the negative consequences of COVID-19 for their firm, cut costs, and take other efficiency measures. Table 4 shows additional examples of these discussions. Throughout 2020, the cost reductions category appears in 11.22 percent of the sentence triples. Turning to financial adjustments, a concern that becomes more prominent from the second quarter of 2020, we classify 13.30 percent of sentence triples in this category. Discussions of financial adjustments are relatively infrequent in the first quarter but represent a meaningful category afterward, only slightly easing in the final month of 2020 .

A relatively small percentage of sentence triples (viz., 1.57 percent) discusses issues regarding government interventions to support the economy or counter the adverse economic effects of the pandemic. Thus, when call participants discuss programs such as the CARES Act or the Paycheck Protection Program, this counts towards the government assistance channel score. ${ }^{32}$

To recapitulate, three findings stand out in Figure 5. First, the discussion of supply and demand impacts is approximately balanced throughout the sample period. Collectively, these direct impacts of the pandemic account for $73.92 \%$ of the specific COVID-19-related concerns raised by call participants. Second, concerns relating to supply chains, specifically, diminish in the second half of 2020 as discussions shift more towards impacts on production and operations and to responses to the pandemic. Third, the discussion of financial adjustments becomes more pronounced in the second quarter and remains stable thereafter. Overall, these latter findings do not suggest an impending widespread financial crisis is taking hold.

Appendix Figure 5 shows the same graph but now also includes the share of other or

\footnotetext{
${ }^{32}$ One possible reason for the relative absence of discussions of government assistance in sentence triples is that many programs were targeted at smaller firms, rendering many of the (relatively large) listed firms in our sample ineligible.
} 
unspecified COVID-19 discussions that can not be specifically attributed to one of our five channel categories. One takeaway from this figure is that the first quarter of 2020 features more conversations about COVID-19 without touching on specific concerns; during this period, call participants typically voice generic uncertainty about what will happen next. Over time, as the impact of the pandemic unfolds over the following quarters, this "unspecified" category shrinks as more and more discussions are tied directly to one of the five channel categories. Indeed, by the end of our sample period, we can allocate about $60 \%$ of sentence triples to a specific channel, up from around $40 \%$ at the beginning of the pandemic.

Once again, drilling down into these aggregate findings offers additional insights. In Figure 6, we partition by geography and by sector, respectively, and show the relative importance of (1) demand versus supply impacts (Panel A) and (2) discussion of financial adjustments relative to all other specific channels (Panel B). ${ }^{33}$ In Panel A, values larger than unity on the scale indicate discussion of demand impacts exceeding discussion of supply impacts. Mentions of financial adjustments are always fewer than mentions of the other four specific categories and, hence, the scale in Panel B marks only values below 1.

Notably, the balance between discussions of supply and demand impacts appears roughly similar across regions worldwide. In Asia, and to a lesser extent in Europe, discussions of COVID-19 are slightly more demand-related. In contrast, supply and demand impacts are more balanced in North America and other regions. Supply impacts are a prominent force at the sector level in the basic materials, healthcare, and energy and utilities sectors, likely reflecting the increased difficulty of production and sourcing in these industries. By contrast, demand impacts are relatively more important for the technology sector: consistent with the view that the pandemic has accelerated the trend toward digital solutions in many areas, such as remote work and online retail.

Financial adjustments to the pandemic are more prominent in earnings-call discussions in Europe and the rest of the world than in Asia or China. Interestingly, whereas the healthcare

\footnotetext{
${ }^{33}$ For additional details for each sector and region, please refer to Appendix Figure 6.
} 
and energy and utilities sectors have similar relative supply impacts, they are on opposite ends concerning their concern with financial adjustments. For healthcare firms, only a few transcripts feature COVID-19-related discussions of access to credit or liquidity. In contrast, the conversation turns to these issues much more frequently for the energy and utilities, academic services, and transportation sectors.

While documenting extensive variation in how the COVID-19 pandemic affects different parts of the economy, these by-sector figures still mask substantial heterogeneity between firms within a given sector. We illustrate this point in Figure 7, which plots the variation of relative demand and supply impacts (Panel A) and relative discussion of financial adjustments (Panel B) for the subsample of S\&P 500 firms. In Panel A, while there is significant clustering around unity, consistent with the observation that the COVID-19 pandemic provides a shock to demand and supply, some firms still stand out.

Linking back to the underlying text fragments used to compile the relative impact scores provides further details. Among firms with relatively high demand impacts, Kinder Morgan's executives, in its April 2020 earnings call, answered the following in reply to a financial analyst's question: "I mean this is certainly different, unprecedented when you put the combination of 2 things, the OPEC Plus falling apart on March 6 together with COVID crushing demand." Or Archer Daniels Midland's management, in July 2020, looking back on the previous months, said that: "... we see that the worst of the demand destruction due to COVID was behind us." Moving to the opposite end of the scale shows firms coping with relatively larger supply impacts. Deere \& Co's management, for example, in the February 2020 earnings call, discusses how they "are monitoring the coronavirus situation and working closely with the Chinese provincial authorities primarily focused on the well-being of our employees and a safe return to production. In terms of overall exposure, the biggest potential impact to Deere is in relation to the supply base that serves our international operations. And the result of those 2 things will certainly impact what we're able to produce and ship during the month." 
Panel B of Figure 7 hones in on the relative discussion of financial adjustments at S\&P 500 firms. Discussions in these firms' earnings calls vary from a simple statement by Delta in April: "The call today will focus on our response to Covid 19, with Ed giving an overview of our priorities and Paul giving an extensive liquidity update" to Coca-Cola Company in the same month discussing the position of bottling partners: "I know they are proactively taking steps to preserve cash, strengthening their balance sheets and manage their P\&Ls. Currently, we don't have any major concerns surrounding our bottling partners from a liquidity perspective, and we are working closely with them to anticipate and deal effectively with a scenario where the coronavirus situation is longer and more severe than currently anticipated." By November 2020, Marathon Oil started discussing the company's dividend policy again: "We would also be well-positioned for incremental return of capital to shareholders beyond our base dividend. Rest assured, we will continue to manage COVID-19 risk diligently through our business continuity and emergency response plans. In a low growth ... environment, capital efficiency ... are the competitive differentiators." At the low end of the scale, the manufacturer of private label over-the-counter pharmaceuticals, Perrigo, had a denominator boost due to their ability to quickly manufacture hand sanitizer in the first quarter of 2020.

It is instructive to compare the relative demand, supply, and financing discussions due to COVID-19 with other disease outbreaks. Figure 8 offers such a comparison between COVID-19, Ebola, SARS, H1N1, Zika, and MERS. We constrain the comparison to the first three quarters after the initial outbreak of each disease. Two observations are striking. For COVID-19, demand and supply-related discussions receive almost the same attention, justifying the view that the 2020 pandemic represents a shock to both supply and demand. The remaining outbreaks mention fewer supply-related concerns and tend to skew more towards demand-side impacts. Interestingly, the outbreaks rank roughly in the order of their severity. Compared with Figure 1, larger aggregate firm-level exposure (where COVID-19 is discussed relatively more frequently than SARS, H1N1, Zika, and MERS, respectively) 
correlates with relatively more severe supply-side impacts. The only disease breaking this pattern is Ebola, which is not discussed as frequently as SARS and H1N1 but has relatively more discussion of supply-side impacts.

Juxtaposing the channels through which these outbreaks affect the economy further underlines that COVID-19 had a disproportionate supply-side impact compared to all earlier epidemics in our sample period. Policymakers seeking to get the economy back on track should question the efficacy of generic, non-targeted stimulus packages to achieve this aim. In some sectors, the demand impact of COVID-19 was sorely felt. Compared with other outbreaks, supply problems have ground down operations and inaugurated significant disruption in other sectors. In the next section, we dig deeper into the problem of determining whether exogenous shocks to demand and supply are net positive or negative at the firm level. ${ }^{34}$

\subsection{Comparing Alternative Methods to Discover Channel Categories}

Before moving our attention to isolating COVID-19-related supply and demand shocks, we pause to compare and contrast our supervised pattern-based classifier with alternative ('offthe-shelf') text classification algorithms.

To this end, we create an additional test set of 250 randomly drawn sentence triples mentioning COVID-19. Two members of the author team classify each text fragment into whether it is about 'demand impacts,' 'supply impacts' (again split into 'production and operations' and 'supply chain'), or 'remainder.' As before, each sentence triple can have

\footnotetext{
${ }^{34}$ The comparison with earlier diseases is important for another reason, as revealed by our systematic analysis of text fragments: namely, these earlier outbreaks are frequently invoked when discussing the impact of COVID-19. For example, in its January 2020 call, as mentioned above, Coca-Cola Company's executives continue: " $\ldots$ to roll out Costa? And also maybe any update or reminder of kind of what SARS did to numbers, if anything, 10 years ago or 15 years ago?" Or, consider HCA Healthcare which opens its earnings call in January 2020 with the assessment that "[h]istorically, SARS or MERS, which are members of the coronavirus family but far more toxic than the current novel coronavirus, did not affect our emergency department volumes." Similarly, analysts ask Prudential Financial: "As we look back to SARS over 15 years ago in light of the coronavirus, do you see any increased demand for your products on the benefit side to note." In sum, the discussions between analysts and executives about their firms' exposure to COVID-19 suggest that firms might have learned from their earlier experience with outbreaks of infectious diseases. This experience could plausibly add to their resilience in the face of the new shock.
} 
multiple category labels.

We then use the same training data of 600 sentence triples that we used to train our pattern-based classifier to train three alternative multiclass classifiers: (1) Naive Bayes, (2) Logistic Regression, and (3) Feedforward Neural Network (Jurafsky and Martin, 2009; Goldberg and Hirst, 2017; Goodfellow et al., 2016; Hastie et al., 2013). Once trained, we compare the out-of-sample performance of these three alternative classifiers with our patternbased algorithm. Our preferred evaluation metric is the $F 1$ score, which trades off precision and recall, where precision is defined as the number of true positives among all positives, and recall is the number of true positives among all sentence triples with the class label (true and false positives). In particular, for a given category such as 'demand impacts,' the F1 score is the (geometric) average of precision and recall for classifying that category (Manning and Schutze, 1999). We summarize each classifier's performance by taking its average F1 score across categories.

We report the results of our comparison in Table 5. The table shows the pattern-based classifier's precision, recall, and F1 score, and the same scores for the three alternative text classification algorithms. The highest performance for each criterion is presented in boldface. Our pattern-based algorithm outperforms the alternative classifiers. Its F1 score is 0.69 , which is substantially higher than the second-best performing classifiers, Naive Bayes and Logistic Regression (F1 score=0.47 for both), closely followed by Feedforward Neural Network $(\mathrm{F} 1$ score $=0.46)$.

The table also suggests that Logistic Regression does best on precision at the cost of having the worst recall. Thus, in our setting, if, for example, we were predominantly worried about the model mislabeling a sentence triple (false positive) as opposed to missing a relevant sentence triple (false negative), Logistic Regression would be a slightly better choice with a precision of 0.76 as opposed to 0.72 from our pattern-based classifier. However, to work well at the earnings-call level, we also need to worry about missing true positives for the 'demand' and 'supply' categories, and the pattern-based algorithm clearly strikes the best 
trade-off.

In sum, this test strongly supports our intuitive, pattern-based method over these more established but also data-hungry machine-learning techniques. We do not, however, believe that these statistics conclusively demonstrate the superiority of our approach in all circumstances. Rather, they underpin our point that when studying a shock in real time, labeled training data to learn patterns in the text reliably are scarce. ${ }^{35}$ With more training data, the performance displayed by alternative methods might improve as the time following the event of interest progresses. In its absence, however, our approach offers a feasible alternative that performs well on a small training set and also enables sensible economic interpretations of the word patterns used.

\section{Shocks: Signing Firm-level Supply and Demand Impacts}

\subsection{Isolating Shocks to Firm-Level Demand and Supply}

So far, our focus has been to summarize our results in channel fractions, showing, in the aggregate, how supply, demand, and financing-related discussions vary for firms across countries, in different sectors, and over time. In what follows, we take the logical next step and exploit the unique feature of our method that reveals, at the firm level, whether COVID-19 is a shock to the supply side or the demand side of the firm. This undertaking is important because it shows how our approach can help inform theories about how demand and supply factors explain changes in stock valuations and other first-order economic quantities, such as corporate investment and hiring.

We must implement one further refinement to our approach before attempting such an investigation. Recognizing that COVID-19 was a boon for some firms and a bane to others, we must "sign" the shock to identify whether the pandemic improved or deteriorated demand for the firm. This signing can also be done for the supply impact category, but practically

\footnotetext{
${ }^{35}$ Many of the commonly-used datasets for text classification-such as the AG news corpus or the DBpedia dataset - have at least a few thousand labeled examples per class (Del Corso et al., 2005; Gulli, 2005; Lehmann et al., 2015).
} 
speaking, few firms experienced positive supply shocks. Hence, we will only consider negative shocks in what follows for this category.

We use the Loughran and McDonald (2011) sentiment dictionary to determine whether negative- or positive-tone words are used in conjunction with discussions of COVID-19's effect on demand. Specifically, for COVID-19-related sentence triples, we count positive minus negative tone words in the sentence triple that identifies the channel and average this across all sentence triples within an earnings-call transcript. Thus, the COVID-19-related net demand shock for a given firm in a given quarter is

$$
\text { COVID-19 net demand shock } k_{i, t}=\frac{1}{S_{i t}} \sum_{s=1}^{S_{i t}}\left\{1\left[s \in \mathbb{P}^{\text {Demand }}\right] \times \text { Tone }(s)\right\} \text {, }
$$

where Tone $(s)$ maps a sentence triple's sentiment words into integer counts. In particular, Tone $(s)$ returns the sum of positive words if the sentence triple contains positive but not negative words; it returns the negative of the sum of negative words if the sentence triple contains negative but not positive words, and it returns zero if the sentence triple contains both positive and negative words. We define COVID-19 negative supply shock ${ }_{i, t}$ similarly but with Tone $(s)$ only returning the integer count of negative sentiment words if the sentence triple that identifies the supply category contains negative but not positive sentiment words.

Table 6 shows how this process works by providing annotated sentence examples. For instance, the snippet from Yokogawa Electric Corp's earnings call transcript, held on 12 May 2020, is identified as a negative demand shock. Highlighted in blue are the words used to determine the category (in this case demand). The sentiment words ("decline" and "suffered"), are highlighted in red, indicating that Yokogawa experienced a negative (demand) shock. The table also contains examples of positive demand and negative supply shocks.

Figure 9 demonstrates, at the sector level, the variation in the extent to which firms exhibit negative net demand (Panel A) and negative supply (Panel B) shocks. As perhaps 
expected by the large-scale restrictions imposed on travel, the largest negative net demand shock is in the transportation sector, while healthcare and the academic and educational services sector, on average, experience much lower negative net demand shocks. Similarly, the real estate and financial sectors exhibit low negative supply shocks compared to the healthcare and industrial goods and services sectors.

\subsection{Valuation Effect of Demand and Supply Shocks}

Having signed the demand and supply shock at the firm level, we examine to what extent the stock market response which we document in Section 2.4 can be attributed to demand and/or supply shocks, respectively. We report regression estimates of annualized quarterly stock returns (in the four quarters of 2020) onto COVID-19 net demand shock ${ }_{i, t}$ and COVID19 negative supply shock ${ }_{i, t}$. Because call participants attribute these shocks to supply and demand directly to COVID-19, we can treat them as exogenous in the classic sense, meaning that, for example, we may assume that a measured negative COVID-19 demand shock affects a firm's valuation through a contraction in demand for the firm's goods, but has no other direct effect on a firm's valuation that is not controlled for by the COVID-19 supply shock or the controls included in the regression. ${ }^{36}$ As before, these controls include the natural logarithm of a firm's total assets in 2019, its market beta in 2018, and a full set of time and sector-fixed effects. We estimate these regressions for the full sample of firms and, separately, for US and large US firms only (as measured by having more than 500 employees).

Table 7 presents the findings. In column 1, we find a positive and statistically significant effect of the net COVID-19 demand shock in the full sample. Specifically, a one-standarddeviation increase in the firm-level net demand shock increases annualized quarterly returns by two percentage points, consistent with the view that a contraction in the firm's demand due to COVID-19 lowers its market valuation. Similarly, when firms are exposed to a negative COVID-19 supply shock, we find a negative effect on annualized quarterly returns $(-0.013$,

\footnotetext{
${ }^{36}$ This interpretation also requires that call participants are largely truthful in their statements and do not engage in systematic deception and cheap talk.
} 
s.e. $=0.006)$, as expected.

Moving from the full sample to our sample of US firms, we find this basic pattern repeated. Comparing the magnitudes of the coefficient estimates for the full and the US sample (in columns 1 and 2, respectively) for both the demand and the negative supply shocks reveals a somewhat stronger relation between stock returns and supply shocks in the US than internationally. This pattern, however, results from smaller-sized sample firms since the coefficient estimates for larger-sized US sample firms (reported in column 3) are closer to those reported for the full sample. We conclude that both COVID-19 supply and demand shocks move stock prices in about equal measure on average.

We investigate the heterogeneity of the valuation effect of the COVID-19 demand and supply shocks further in Figure 10, in which we visualize the coefficient estimates (and corresponding $95 \%$ confidence intervals) from the same regression as in Table 7 this time estimated separately for each sector represented in the US sample. The figure shows that the valuation effects in the transportation and real estate sectors are driven predominantly by the demand shock. In contrast, valuation in other sectors like healthcare, consumer goods (both cyclical and non-cyclical), and energy and utilities appear more affected by supply shocks associated with the pandemic.

Validation checks. Our exercise to attribute the valuation effects of COVID-19 to demand and/or supply shocks hinges on the condition that our approach to "sign" the shocks is successful and that our ability to correctly identify positive and negative shocks does not systematically vary. We assess these conditions in two ways. First, we relate our positive and negative demand shocks to the firm's revenue in the 2019 and 2020 calendar years using a 'difference-in-differences' specification in which we think of each firm's intensity of the COVID-19 net demand shock as a continuous firm-level treatment:

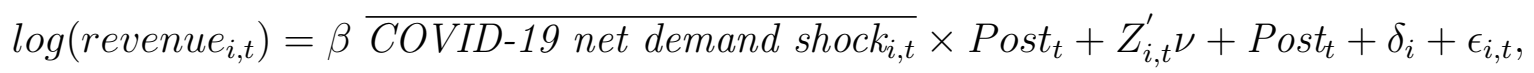


where $\log \left(\right.$ revenue $\left._{i, t}\right)$ represents the $\log$ of sales revenue, Post $_{t}$ is an indicator variable equal

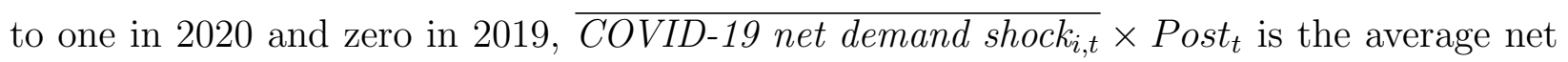
exposure score to the positive and negative COVID-19 demand shock in 2020, $Z_{i, t}^{\prime}$ contains the interaction of the $\log$ of firm $i$ 's total assets in 2019 and Post $_{t}$, and $\delta_{i}$ represents firm fixed effects.

Panel A of Table 8 reports the results. In column 1, we find a clear and highly statistically

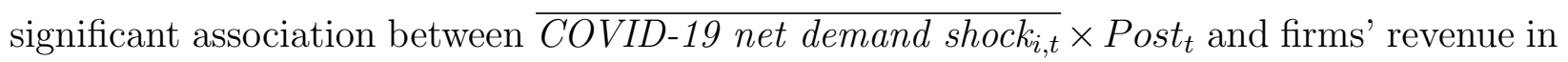
2020 (0.029, s.e. $=0.005)$. More importantly, in column 2, where we distinguish positive and negative COVID-19 demand shocks, we find a significant positive coefficient $(0.013$, s.e. $=0.006)$ for the former and a negative coefficient $(-0.020$, s.e. $=0.005)$ for the latter. Both associations are approximately the same size - we cannot reject the null that they are equal to each other. In particular, a one-standard-deviation increase in the positive (negative) demand shock is associated with a $1.3 \%(2.0 \%)$ decrease in revenue, respectively. ${ }^{37}$

As an additional means of probing for differential measurement error, we conduct a human audit of our ability to correctly identify the sentiment discussions of COVID-19's supply and demand impacts. As part of this audit, we assigned team members to read one hundred sentence triples for each category (positive demand, negative demand, negative supply). They were asked to determine whether each sentence triple is correctly classified and, if so, has recorded the correct sentiment. We find that the rate of true positives that satisfy these two conditions is very similar for the three categories. Panel B of Table 8 reports the details. Specifically, the rate of true positives is $0.67,0.65$, and 0.57 for COVID-19 negative supply shock, COVID-19 negative demand shock, and COVID-19 positive demand shock, respectively. These true positive rates are somewhat lower than the precision reported in Table 5 as the current test requires the correct classification of both categories and sentiment. Using a normal approximation of the observed proportions test, the $p$-value for the null

\footnotetext{
${ }^{37}$ Ideally, we would like to follow a similar approach to validate the identification of negative supply shocks, but in the absence of a compelling dependent accounting variable that could capture the economic consequences on the firm's supply-side from the COVID-19 event, we defer following up on this idea.
} 
hypothesis of equal measurement error across the three categories is never below 0.15 . Thus, we conclude that we can reliably "sign" shocks and that any differential measurement error for negative and positive shocks is too small to detect statistically.

\subsection{Firm Outcomes after Demand and/or Supply Shocks}

Having shown that our text-based method to identify a firm's exposure to demand and supply shocks is useful for understanding the valuation effects of the COVID-19 pandemic, we take one further step and examine the importance of demand and supply factors to explain changes in firms' investment and hiring during the COVID-19 crisis. We return to the 'difference-in-differences' set-up introduced in equation 4 but now replace the dependent variable $\log \left(\right.$ revenue $\left._{i, t}\right)$ with the average investment rate $i_{i, t} / k_{i, t}$ of firm $i$ in year $t$, calculated according to the inventory method (Stein and Stone, 2013), and with employment $t_{i, t}$, which is the log of the number of employees (in thousands). We report results for the full sample and the sample of US firms separately in Table 9. In Panel A, we detail the estimation results using the investment rate as the dependent variable, whereas Panel B reports the findings for employment.

We find that the intensity of a firm's COVID-19 net demand shock in 2020 has a significant positive effect on its investment, again consistent with the view that firms that suffer a negative demand shock due to COVID-19 invest less. In column 1, for the full sample, the estimated coefficient equals 0.037 (s.e. $=0.013)$, implying that a one-standard-deviation decrease in a firm's COVID-19 net demand shock decreases the firm's investment by 3.7 percent. Column 2 in Panel A suggests that for US firms, the impact of the pandemic on investment is almost twice as strong compared to the full sample (possibly because the US sample also includes relatively smaller-sized firms that suffered larger declines in their investment). Column 3 focuses on large US firms, for which we again obtain a coefficient estimate similar to the full sample $(0.033$, s.e.=0.016). In contrast, the firm's exposure to supply shocks does not have a noticeable differential effect on investment when comparing 
2019 and 2020. This conclusion is true for the full sample and US firms only.

This asymmetry in investment responses is intriguing, given that both shocks appear to be priced in about equal measure. Moreover, a wide range of models predicts investment should respond similarly to contractions of supply and demand (this is particularly true for a standard neoclassical model with shocks to total factor productivity and a CES demand structure; e.g., Hsieh and Klenow (2009)). One possibility is that firms expected COVID19's demand shock to be much more persistent than its supply impacts, as suggested more generally by Foster et al. (2008).

When considering employment in Panel B, we again find a positive, albeit not statistically significant, effect of the net demand shock in the full sample and a negative marginally significant effect of the negative supply shock, implying that firms affected by the negative supply shock hire less. In column 2, both coefficients of interest are statistically indistinguishable from zero for the full sample of US firms. However, column 3 shows significant demand and supply shock effects for large US firms. A one-standard-deviation increase in negative demand and supply shock lowers employment at these large firms by $1.9 \%$ and 1\%, respectively. This pattern of differential responses by small and large firms may well arise because the US Paycheck Protection Program paid small but not large firms to preserve jobs throughout the pandemic.

Taking a step back, our findings on the significant effects of the COVID-19 demand shock on firm-level stock market valuations, investment, and (to a lesser extent) hiring have important implications for ongoing policy debates. In particular, our results suggest that policy measures designed to support demand for goods and services (such as loose monetary and fiscal policy) can mitigate the significant negative effects of the COVID-19 pandemic on firm-level investment and employment that stem from a contraction in demand. By contrast, adverse effects arising from supply-side impacts, particularly in the labor market, may be harder to address. ${ }^{38}$

\footnotetext{
${ }^{38}$ We show in Appendix Figure 7 evidence supporting the parallel trends assumption for these results. For the specification in column 3 of Table 9, the figure shows the percentage change in the investment rate
} 


\section{Conclusions}

The economic fallout from the worldwide spread of COVID-19 has made clear the need to understand better the firm-level impact of such large economic shocks in real time. Data on how the pandemic affects firms, sectors, and regions are key for effective policy responses and understanding how its effects propagate through supply chains and across borders.

In this paper, we provide measures of the exposure of individual firms to epidemic diseases. We do so based on transcripts of quarterly earnings conference calls held by a large sample of listed firms around the globe. Using these earnings-call transcripts, we can not only measure the risk and sentiment each firm associated with the disease, but we also introduce a new automated text-based pattern discovery method to systematically extract information about the nature of the cardinal issues firms face as they respond to the challenges of the pandemic. Ultimately, combining this investigation of channels with sentiment analysis of the surrounding text, we are able to measure directly the firm-level supply and demand shocks attributable to the COVID-19 pandemic.

Using the toolbox developed in this paper, we report salient statistics on how the COVID19 pandemic impacted the global economy in 2020. Perhaps the best way to summarize these findings is that the coronavirus impacted countries, industries, and firms in a multifaceted way with large differences in how the episode played out at each level of analysis.

Our ability to pinpoint the relative importance of demand and supply shocks related to the COVID-19 pandemic for each firm and the timely measurement of the firm's exposure (as firms host these earnings calls every quarter), renders the data potentially well-suited for testing theories in finance and economics, where identification is often challenging. As we learn about the channels through which shocks operate, as perceived by managers and noncorporate call participants, we gain the power to attribute firm-level outcomes to (exogenous) demand and supply shocks related to the pandemic. We document how these factors explain

(employment) associated with firms facing a one-standard-deviation higher COVID-19 demand shock on the vertical axis in Panel A (Panel B). We find no significant coefficient estimates in any year (starting from 2016) before 2020. 
important firm outcomes such as investment and hiring. Thus, our methodological advance provides a versatile way forward to produce new granular data that can inform on issues of key importance to investors, policymakers, and researchers alike.

One key takeaway from this analysis is that demand shocks stemming from the COVID-19 pandemic have significantly depressed firms' stock valuations, investment activities, and (to a lesser extent) their employment. Part of the economic crisis in the wake of the pandemic is thus clearly attributable to shortfalls in demand, which can be addressed with appropriate monetary and fiscal policy. For an economically significant part of the economy, however, supply distortions, rather than the demand impact, caused problems. Stimulus policies are much less likely to be effective for these sectors. 


\section{REFERENCES}

Acemoglu, D., A. Ozdaglar, and A. Tahbaz-Salehi (2017). Microeconomic origins of macroeconomic tail risks. American Economic Review 10\%, 54-108.

Alfaro, L., A. Chari, A. N. Greenland, and P. K. Schott (2020). Aggregate and firm-level stock returns during pandemics, in real time. NBER Working paper no. 26950.

Alon, T., M. Kim, D. Lagakos, and M. VanVuren (2020). How should policy responses to the COVID-19 pandemic differ in the developing world? NBER Working paper no. 27273.

Arnon, A., J. Ricco, and K. Smetters (2020). Epidemiological and economic effects of lockdown. Brookings Papers on Economic Activity.

Atkeson, A. (2020). What will be the economic impact of Covid-19 in the US? Rough estimates of disease scenarios. NBER Working paper no. 26867.

Au, S.-Y., M. Dong, and X. Zhou (2020). Does social interaction spread fear among institutional investors? Evidence from Covid-19. Working paper, York University.

Baker, S., N. Bloom, S. Davis, K. Kost, M. Sammon, and T. Viratyosin (2020). The unprecedented stock market reaction to COVID-19. Review of Asset Pricing Studies 10, $742-758$.

Baker, S. R., N. Bloom, and S. J. Davis (2016). Measuring economic policy uncertainty. The Quarterly Journal of Economics 131 (4), 1593-1636.

Baqaee, D. and E. Farhi (2019). The macroeconomic impact of microeconomic shocks: Beyond Hulten's theorem. Econometrics 87, 1155-1203.

Baqaee, D. and E. Farhi (2020). Nonlinear production networks with an application to the covid-19 crisis. NBER Working paper no. 27281.

Barrero, J. M., N. Bloom, S. J. Davis, and B. H. Meyer (2021, May). Covid-19 is a persistent reallocation shock. AEA Papers and Proceedings 111, 287-91.

Barro, R. J., J. F. Ursúa, and J. Weng (2020). The coronavirus and the great influenza pandemic: Lessons from the "spanish flu" for the coronavirus's potential effects on mortality and economic activity. NBER Working paper no. 26866.

Barrot, J.-N. and J. Sauvagnat (2016). Input specificity and the propagation of idiosyncratic shocks in production networks. The Quarterly Journal of Economics 131(3), 1543-1592.

Bekaert, G., E. Engstrom, and A. Ermolov (2020). Aggregate demand and aggregate supply effects of COVID-19: A real-time analysis. Available at SSRN 3611399.

Blanchard, O. and D. Quah (1989). The dynamic effects of aggregate demand and supply disturbances. American Economic Review 79(4), 655-673.

Boehm, C. E., A. Flaaen, and N. Pandalai-Nayar (2019). Input linkages and the transmission of shocks: Firm-level evidence from the 2011 Tōhoku earthquake. Review of Economics and Statistics 101(1), 60-75.

Bretscher, L., A. Hsu, P. Simasek, and A. Tamoni (2020). Covid-19 and the cross-section of equity returns: Impact and transmission. The Review of Asset Pricing Studies 10(4), $705-741$. 
Bybee, L., B. Kelly, A. Manela, and D. Xiu (2019). The structure of economic news. Working paper.

Caldara, D., M. Iacoviello, P. Molligo, A. Prestipino, and A. Raffo (2019). The economic effects of trade policy uncertainty. Journal of Monetary Economics.

Carvalho, V., M. Nirei, Y. Saito, and A. Tahbaz-Salehi (2021). Supply chain disruptions: Evidence from the Great East Japan Earthquake. Quarterly Journal of Economics 136, $1255-1321$.

Croce, M. M. M., P. Farroni, and I. Wolfskeil (2020). When the markets get COVID: Contagion, viruses, and information diffusion. VID: Contagion, Viruses, and Information Diffusion (March 23, 2020).

Davis, S. J., S. Hansen, and C. Seminario-Amez (2021). Firm-level risk exposures and stock returns in the wake of COVID-19. NBER Working paper no. 27867.

Davis, S. J., D. Liu, and X. S. Sheng (2021). Stock prices, lockdowns, and economic activity in the time of coronavirus. Technical report, National Bureau of Economic Research.

Del Corso, G. M., A. Gullí, and F. Romani (2005). Ranking a stream of news. In Proceedings of the 14th International Conference on World Wide Web, WWW '05, pp. 97-106. Association for Computing Machinery.

Ding, W., R. Levine, C. Lin, and W. Xie (2021). Corporate immunity to the COVID-19 pandemic. Journal of Financial Economics 141(2), 802-830.

Eichenbaum, M. S., S. Rebelo, and M. Trabandt (2021). The macroeconomics of epidemics. The Review of Financial Studies 34(11), 5149-5187.

Faria-e Castro, M. (2021). Fiscal policy during a pandemic. Journal of Economic Dynamics and Control 125, 104088.

Ferrando, A. (2020). Firms' expectations on access to finance at the early stages of the Covid-19 pandemic. ECB Working paper no. 20202446.

Fogli, A. and L. Veldkamp (2021). Germs, social networks and growth. The Review of Economic Studies 88(3), 1074-1100.

Fornaro, L. and M. Wolf (2020). Covid-19 coronavirus and macroeconomic policy. CEPR Discussion paper no. DP14529.

Foster, L., J. Haltiwanger, and C. Syverson (2008). Reallocation, firm turnover, and efficiency: Selection on productivity or profitability? American Economic Review 98(1), 394-425.

Gentzkow, M., B. Kelly, and M. Taddy (2019). Text as data. Journal of Economic Literature $57(3), 535-74$.

Giglio, S., M. Maggiori, J. Stroebel, and S. Utkus (2021). The joint dynamics of investor beliefs and trading during the COVID-19 crash. Proceedings of the National Academy of Sciences 118(4).

Goldberg, Y. and G. Hirst (2017). Neural Network Methods in Natural Language Processing. Synthesis Lectures on Human Language Technologies. Morgan \& Claypool Publishers. 
Goodfellow, I., Y. Bengio, and A. Courville (2016). Deep Learning. Adaptive Computation and Machine Learning series. MIT Press.

Gormsen, N. and R. Koijen (2020). Coronavirus: Impact on stock prices and growth expectations. Review of Asset Pricing Studies 10, 574-597.

Greenwald, D. L., J. Krainer, and P. Paul (2021). The credit line channel. FRB of San Francisco Working paper.

Greenwood, J., P. Kircher, C. Santos, and M. Tertilt (2019). An equilibrium model of the African HIV/AIDS epidemic. Econometrica 87(4), 1081-1113.

Griffiths, T. L. and M. Steyvers (2004). Finding scientific topics. Proceedings of the National Academy of Sciences 101(suppl_1), 5228-5235.

Guerrieri, V., G. Lorenzoni, L. Straub, and I. Werning (2022). Macroeconomic implications of COVID-19: Can negative supply shocks cause demand shortages? American Economic Review 112(5), 1437-74.

Gulli, A. (2005). The anatomy of a news search engine. WWW '05, pp. 880-881. Association for Computing Machinery.

Handley, K. and J. F. Li (2018). Measuring the effects of firm uncertainty on economic activity: New evidence from one million documents. Technical report, Mimeo., University of Michigan.

Hansen, S., M. McMahon, and A. Prat (2018). Transparency and deliberation on the FOMC: A computational linguistics approach. Quarterly Journal of Economics 133(2), 801-870.

Hassan, T. A., S. Hollander, L. van Lent, and A. Tahoun (2019). Firm-level political risk: Measurement and effects. The Quarterly Journal of Economics 134(4), 2135-2202.

Hassan, T. A., S. Hollander, L. van Lent, and A. Tahoun (2020). The global impact of Brexit uncertainty. NBER Working paper no 26609; INET Working paper no. 106.

Hastie, T., R. Tibshirani, and J. Friedman (2013). The Elements of Statistical Learning: Data Mining, Inference, and Prediction. Springer Series in Statistics. Springer New York.

Hollander, S., M. Pronk, and E. Roelofsen (2010). Does silence speak? An empirical analysis of disclosure choices during conference calls. Journal of Accounting Research 48(3), 531563.

Hsieh, C.-T. and P. J. Klenow (2009). Misallocation and manufacturing tfp in china and india. The Quarterly journal of economics 124(4), 1403-1448.

Jamilov, R., H. Rey, and A. Tahoun (2021). The anatomy of cyber risk. Working paper, London Business School.

Joaquim, G. and F. Netto (2020). Bank incentives and the impact of the Paycheck Protection Program. Available at SSRN 3704518.

Jurafsky, D. and J. Martin (2009). Speech and Language Processing: An Introduction to Natural Language Processing, Computational Linguistics, and Speech Recognition. Prentice Hall series in artificial intelligence. Pearson Prentice Hall. 
Kaplan, G., K. Mitman, and G. L. Violante (2020). The housing boom and bust: Model meets evidence. Journal of Political Economy 128(9), 3285-3345.

Kaplan, G., B. Moll, and G. Violante (2020). The great lockdown and the big stimulus: Tracing the pandemic possibility frontier for the U.S. Working paper, University of Chicago.

Kargar, M., B. Lester, D. Lindsay, S. Liu, P.-O. Weill, and D. Zúñiga (2021). Corporate bond liquidity during the COVID-19 crisis. The Review of Financial Studies 34 (11), 5352-5401.

Kost, K. (2019). Trade policy uncertainty, investment, and lobbying. Working paper, University of Chicago.

Kothari, S. and J. Warner (2007). Econometrics of event studies. Handbook of Empirical Corporate Finance 1, 3-36.

Lehmann, J., R. Isele, M. Jakob, A. Jentzsch, D. Kontokostas, P. N. Mendes, S. Hellmann, M. Morsey, P. van Kleef, S. Auer, and C. Bizer (2015). Dbpedia - a large-scale, multilingual knowledge base extracted from wikipedia. Semantic Web 6(2), 167-195.

Long, J. and C. Plosser (1983). Real business cycles. Journal of Political Economy 91, 39-69.

Loughran, T. and B. McDonald (2011). When is a liability not a liability? Textual analysis, dictionaries, and 10-Ks. The Journal of Finance 66(1), 35-65.

Loughran, T. and B. McDonald (2016). Textual analysis in accounting and finance: A survey. Journal of Accounting Research 54(14), 1187-1230.

Ma, Y., K. Xiao, and Y. Zeng (2020). Mutual fund liquidity transformation and reverse flight to liquidity. Available at SSRN 3640861.

Manning, C. and H. Schutze (1999). Foundations of Statistical Natural Language Processing. MIT Press.

Mian, A., K. Rao, and A. Sufi (2013). Household balance sheets, consumption, and the economic slump. The Quarterly Journal of Economics 128(4), 1687-1726.

Moser, C. and P. Yared (2021). Pandemic lockdown: The role of government commitment. Review of Economic Dynamics.

Nekarda, N. and V. Ramey (2020). The cyclical behavior of the price-cost markup. Journal of Money, Credit and Banking 52, 319-353.

Ozik, G., R. Sadka, and S. Shen (2021). Flattening the illiquidity curve: Retail trading during the COVID-19 lockdown. Journal of Financial and Quantitative Analysis 56(7), $2356-2388$.

Philipson, T. (1999). Economic epidemiology and infectious diseases. NBER Working paper no. 7037.

Ramelli, S. and A. Wagner (2020). Feverish stock price reactions to COVID-19. Swiss Finance Institute Research Paper no. 20-12.

Sautner, Z., L. van Lent, G. Vilkov, and R. Zhang (2022). Firm-level climate change exposure. Journal of Finance, Forthcoming. 
Stein, L. C. and E. C. Stone (2013). The effect of uncertainty on investment, hiring, and R\&D: Causal evidence from equity options. Working paper, Available at SSRN 1649108.

Stephany, F., L. Neuhäuser, N. Stoehr, P. Darius, O. Teutloff, and F. Braesemann (2022). The CoRisk-Index: a data-mining approach to identify industry-specific risk perceptions related to Covid-19. Humanities and Social Sciences Communications 9, 1-15. 
Figure 1: Percentage of earnings calls discussing epidemic diseases

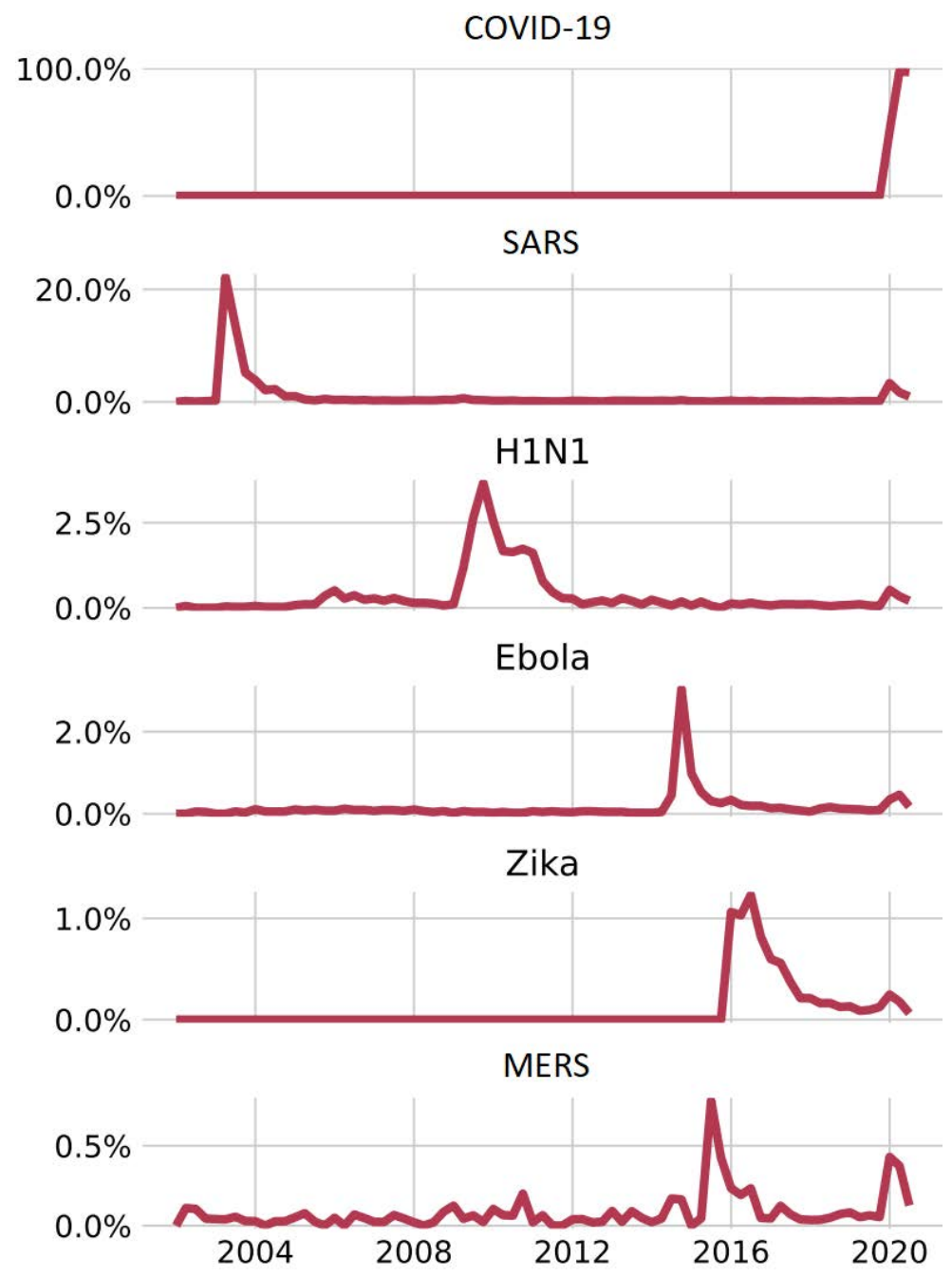

Notes: This figure plots the percentage of earnings calls discussing epidemic diseases (COVID-19, SARS, H1N1, Ebola, Zika, and MERS) by quarter from January 2002 through December 2020. 
Figure 2: Discussion of COVID-19, SARS, and H1N1 in earnings calls by region

(a) Average COVID-19 exposure $_{i, t}$ by region

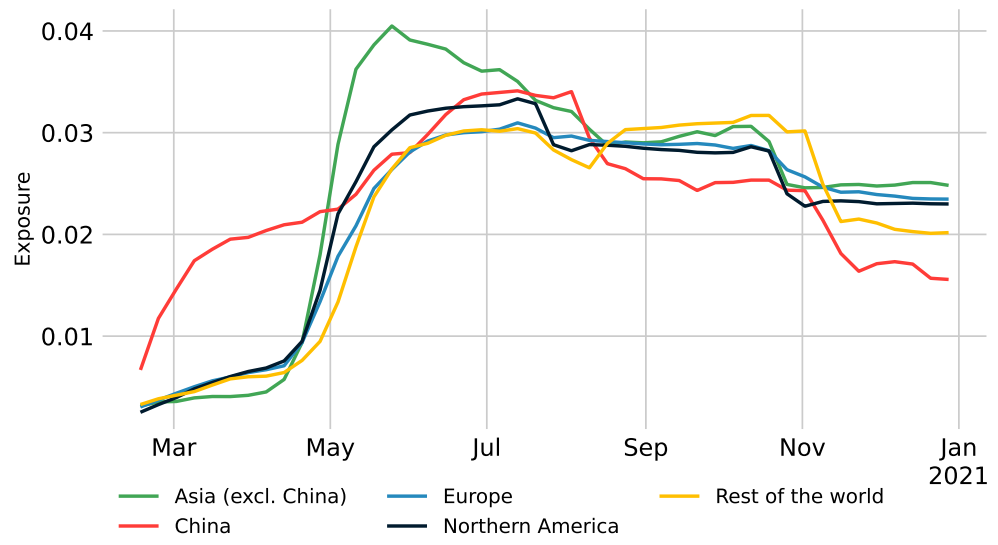

(b) Average $S A R S$ exposure $_{i, t}$ by region

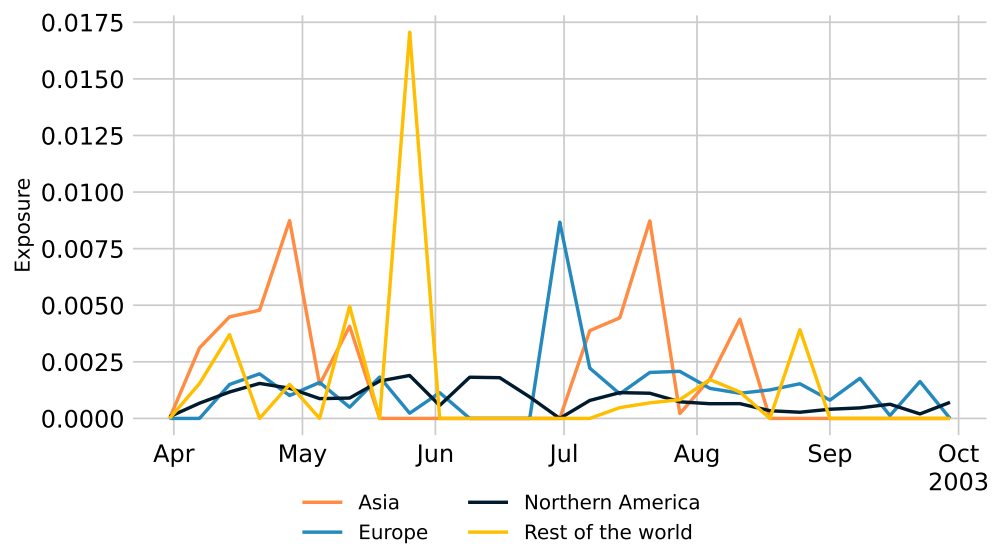

(c) Average $H 1 N 1$ exposure $_{i, t}$ by region

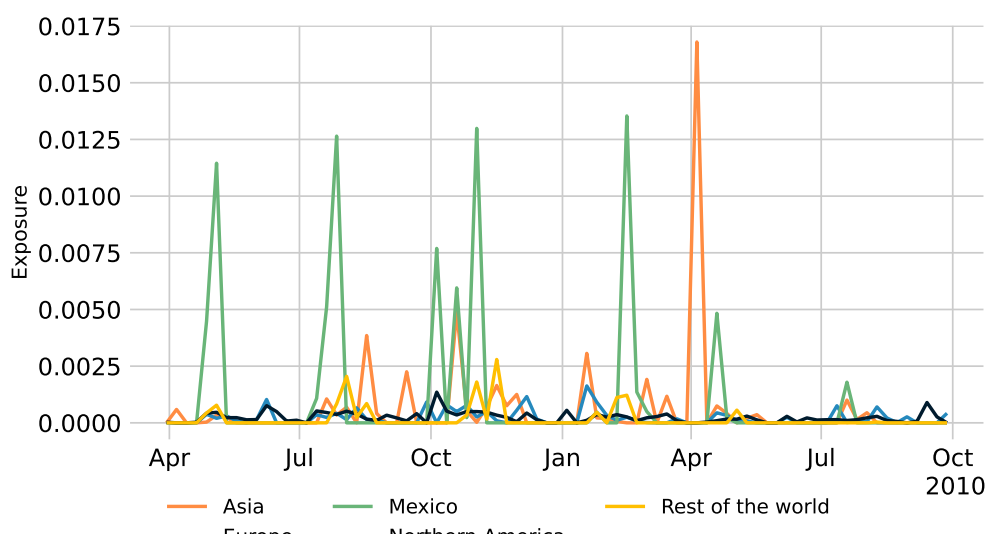

Notes: This figure plots the weekly average of COVID-19 exposure $_{i, t}$, SARS exposure $_{i, t}$, and H1N1 exposure ${ }_{i, t}$ for firms headquartered in the indicated region for the first $7+$ months after the initial disease outbreak. Exposure measures are scaled by the number of sentences in the earnings call transcript. The time series in Panel (a) are smoothed with a weighted moving average over the last 12 weeks using the number of earnings conference calls as weights. 
Figure 3: Weekly average of COVID-19 risk ri,t $_{\text {and COVID-19 sentiment }}$,t

(a) Weekly average of COVID-19 risk $k_{i, t}$

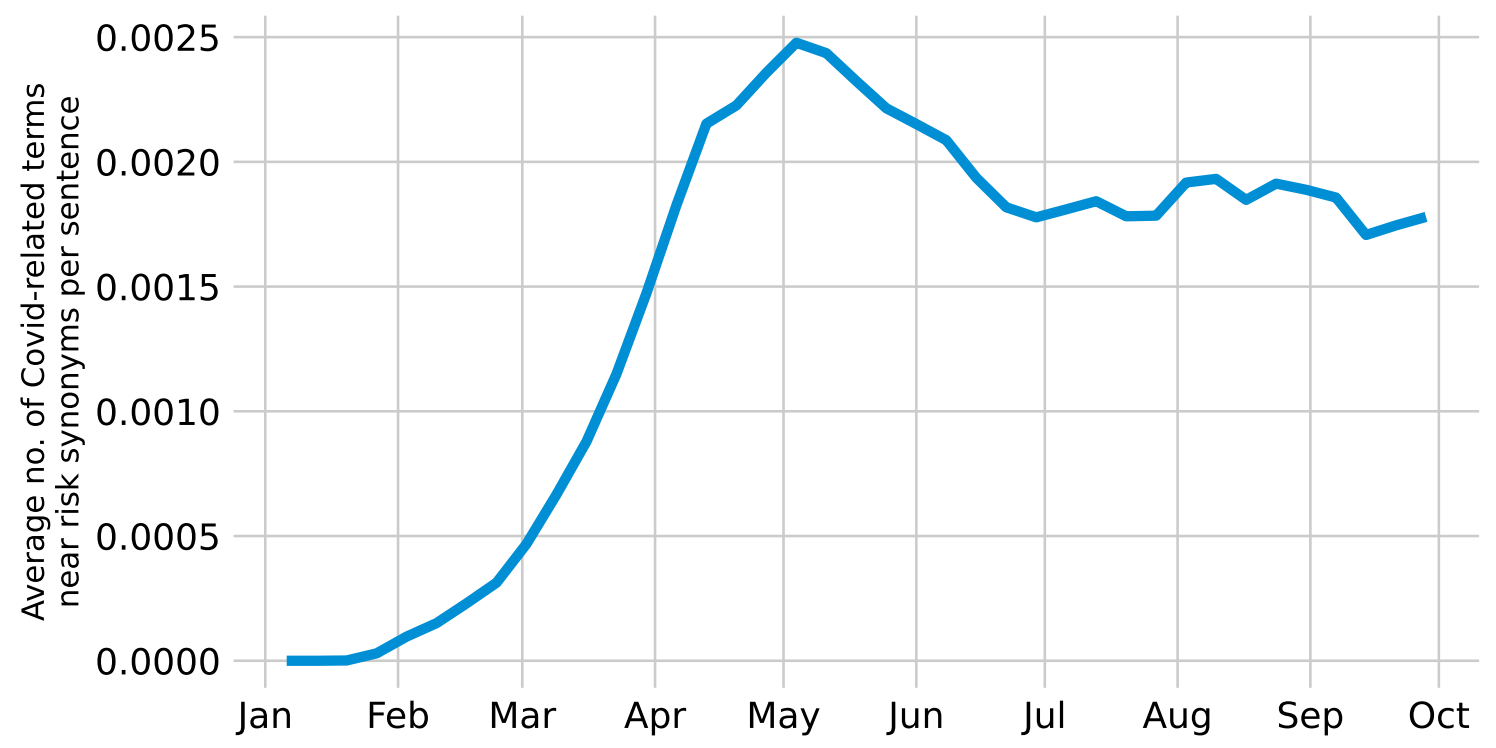

(b) Weekly average of COVID-19 sentiment $t_{i, t}$

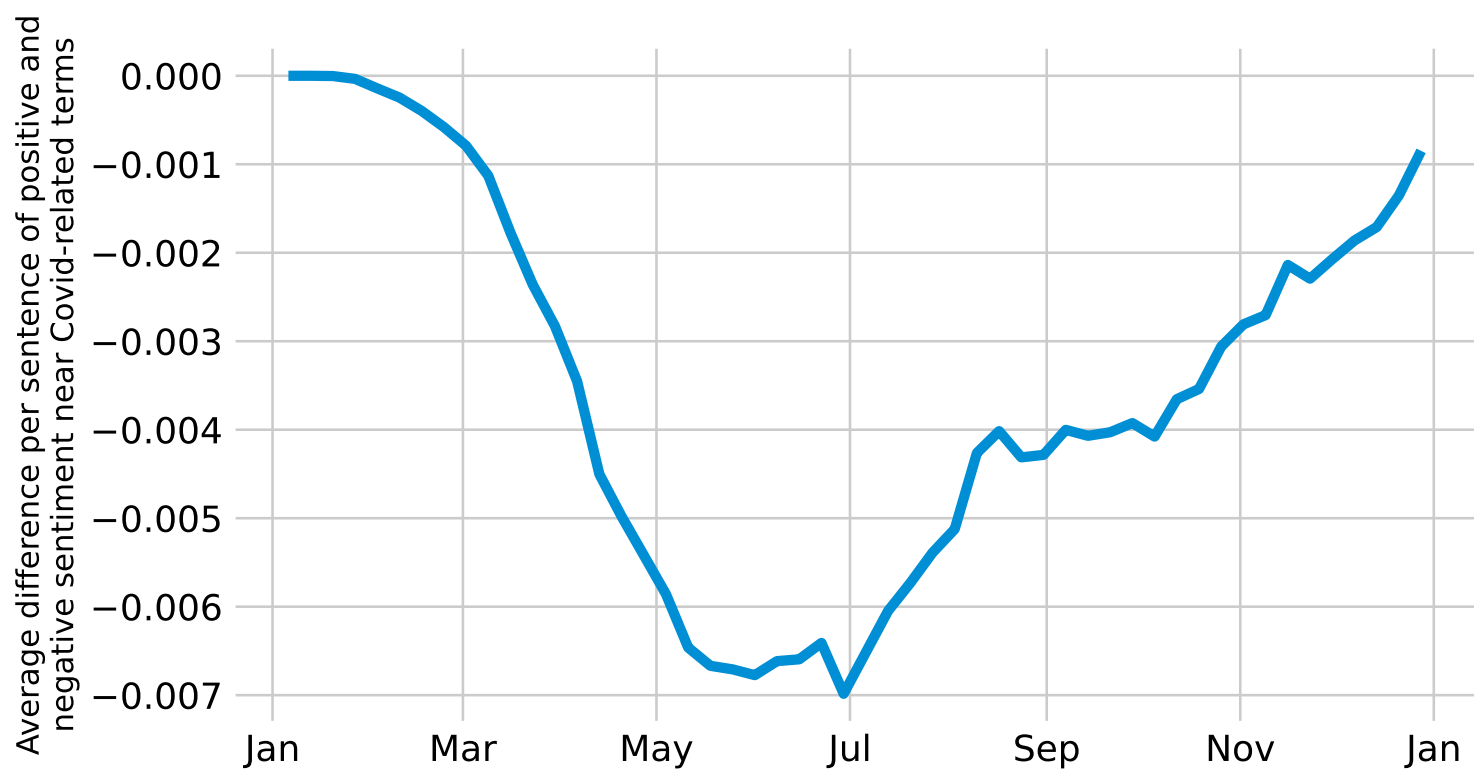

Notes: This figure plots the weekly average of COVID-19 risk $_{i, t}$ and COVID-19 sentiment ${ }_{i, t}$ across all earnings conference calls held from January through December 2020. The time series are smoothed with a moving average over the last 6 weeks with equal weighting. 
Figure 4: Average COVID-19 risk $_{i, t}$ and COVID-19 sentiment ${ }_{i, t}$ by sector

(a) Average COVID-19 risk $i, t$ by sector

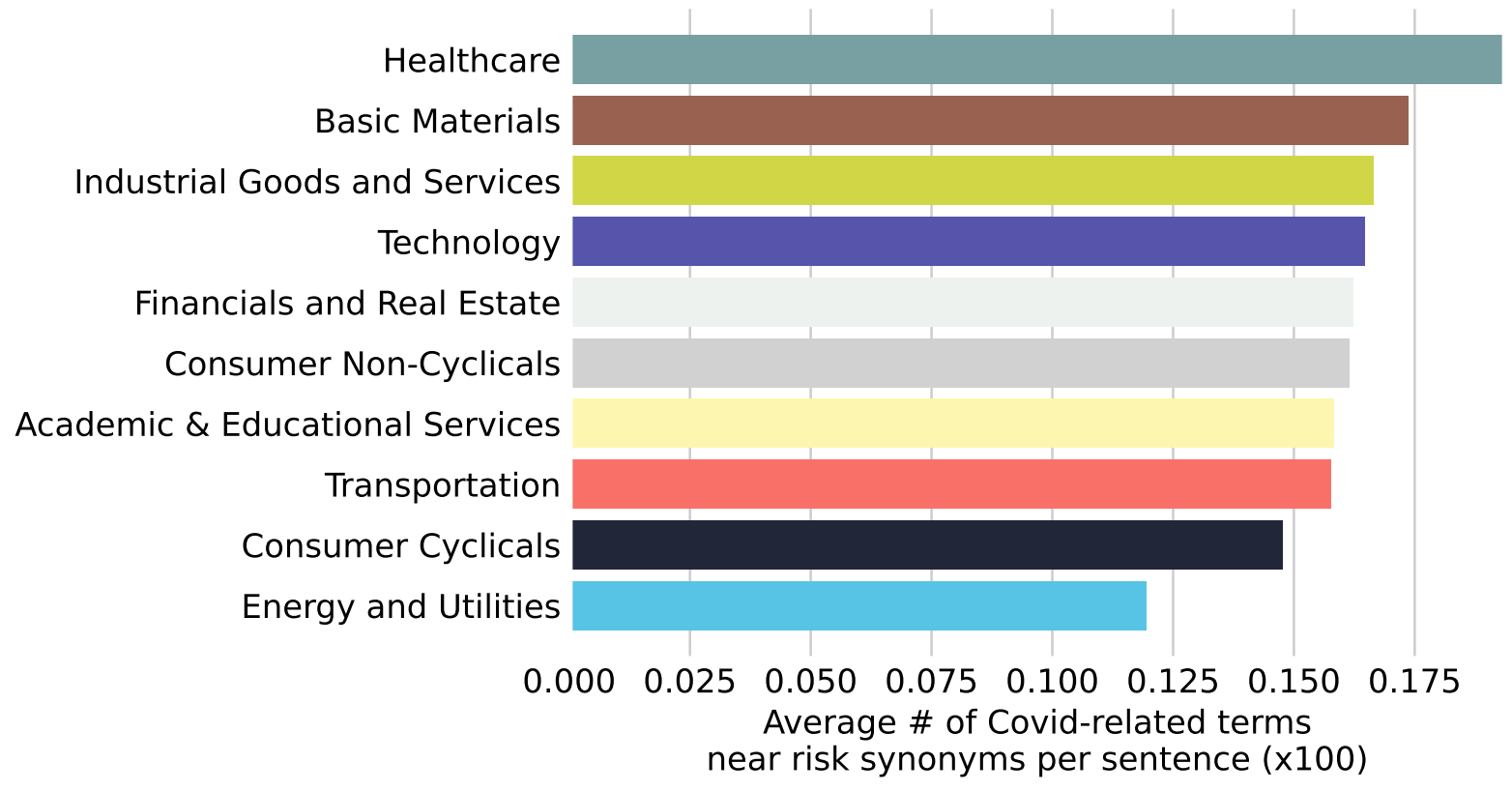

(b) Average COVID-19 sentiment ${ }_{i, t}$ by sector

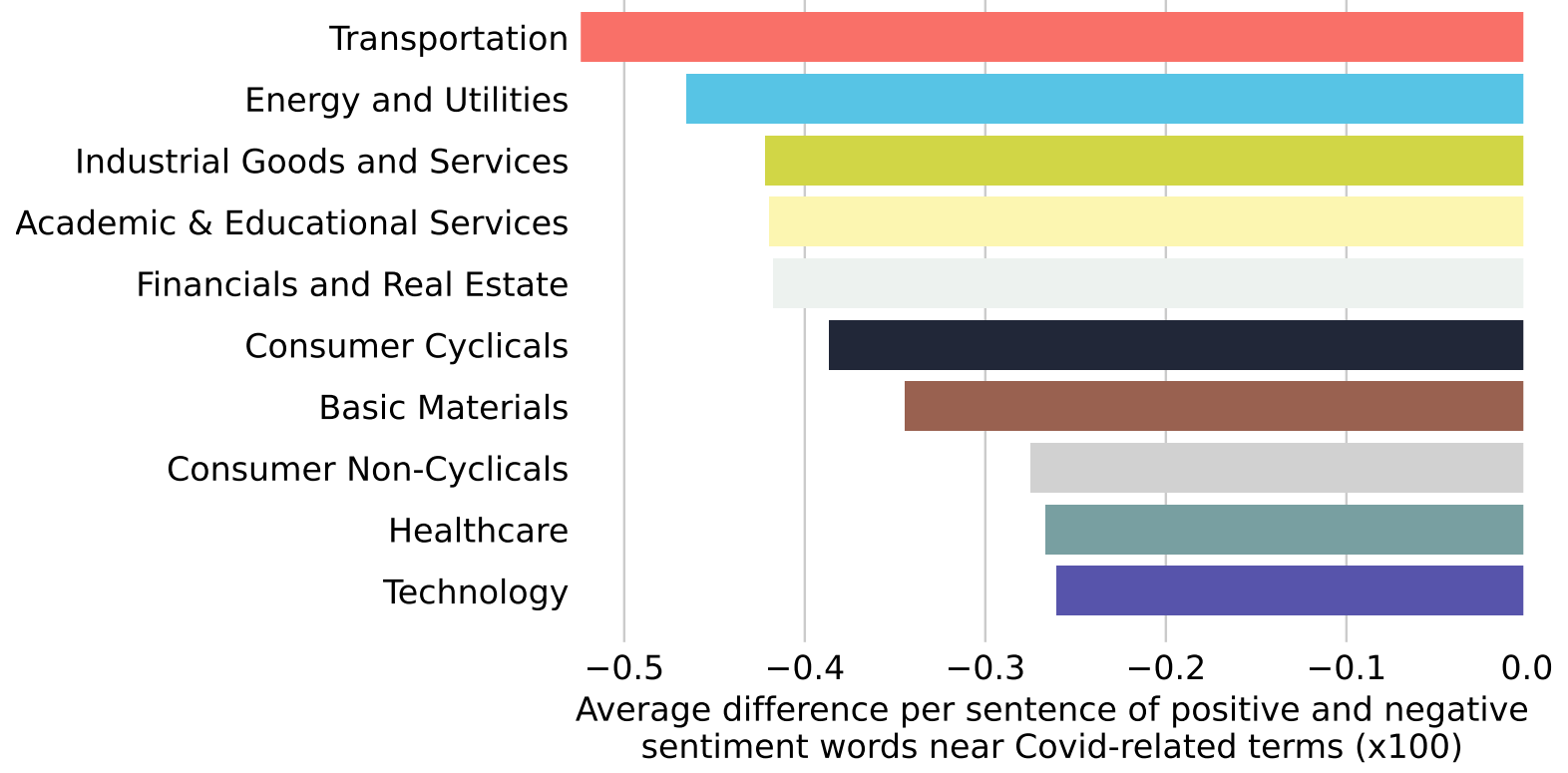

Notes: This figure plots average COVID-19 risk $_{i, t}$ and COVID-19 sentiment $_{i, t}$ by sector across all earnings conference calls held by firms in the indicated sector between January and December 2020. The averages are multiplied by 100 for exposition purposes. The sector classification is based on a firm's "Economic Sector" from the Refinitiv Eikon database. 
Figure 5: Topic decomposition of COVID-19-related speech

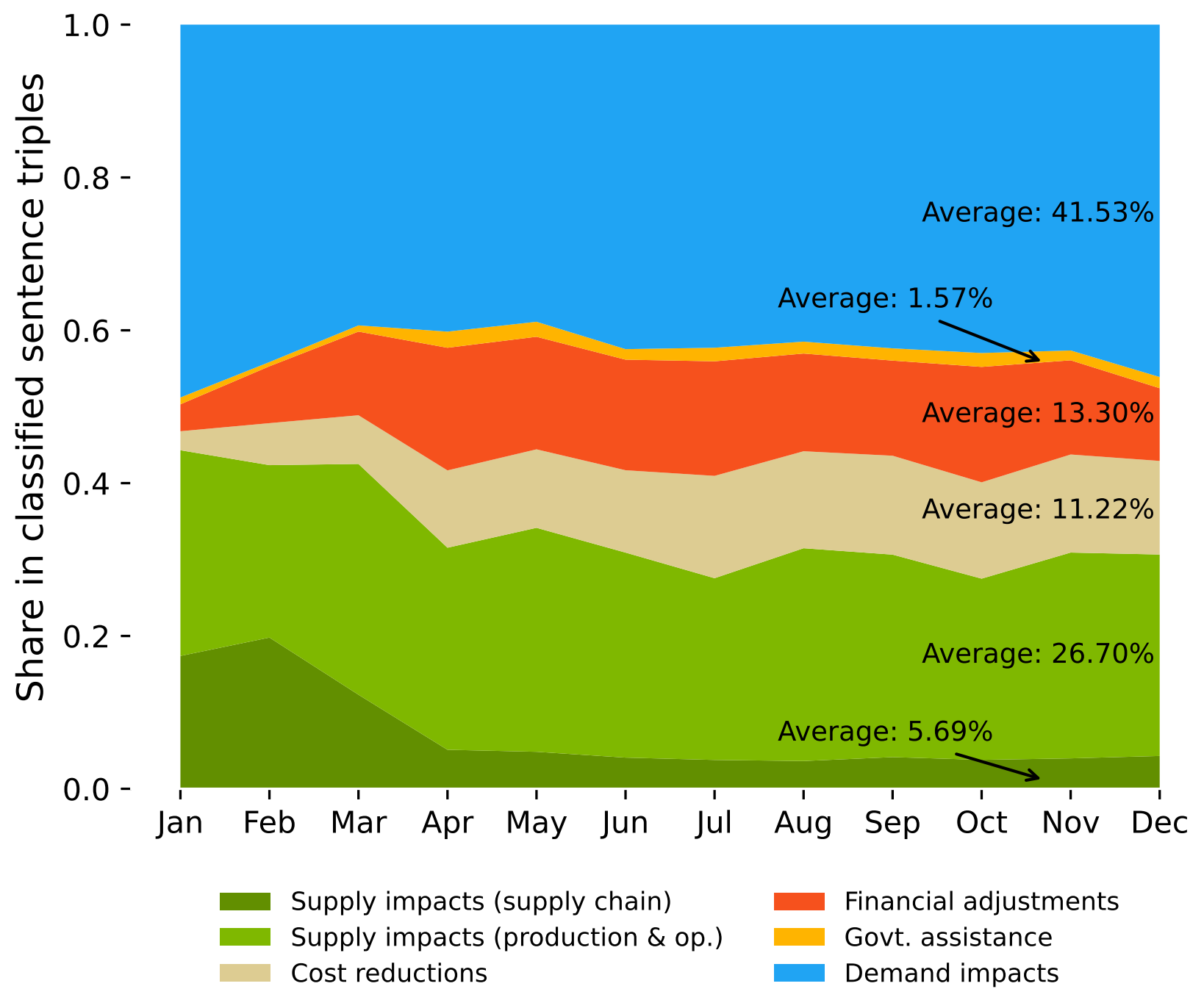

Notes: This figure plots the share of each of five topics ('supply impacts' (i.e., 'supply chain' and 'production and operations'), 'cost reductions,' 'financial adjustments,' 'government assistance,' and 'demand impacts') in classified sentence triples mentioning COVID-19 in transcripts of earnings conference calls held from January through December 2020. A sentence triple is defined as three consecutive sentences (if available) by the same speaker with the middle sentence containing a COVID-19-related keyword. Sentence triples assigned to more than one topic are duplicated for the purpose of determining the denominator; by doing so, shares add up to one. 
Figure 6: COVID-19 demand/supply impacts \& financial/non-financial adjustments by region and sector

(a) Mean COVID-19 demand/supply impacts by region and sector

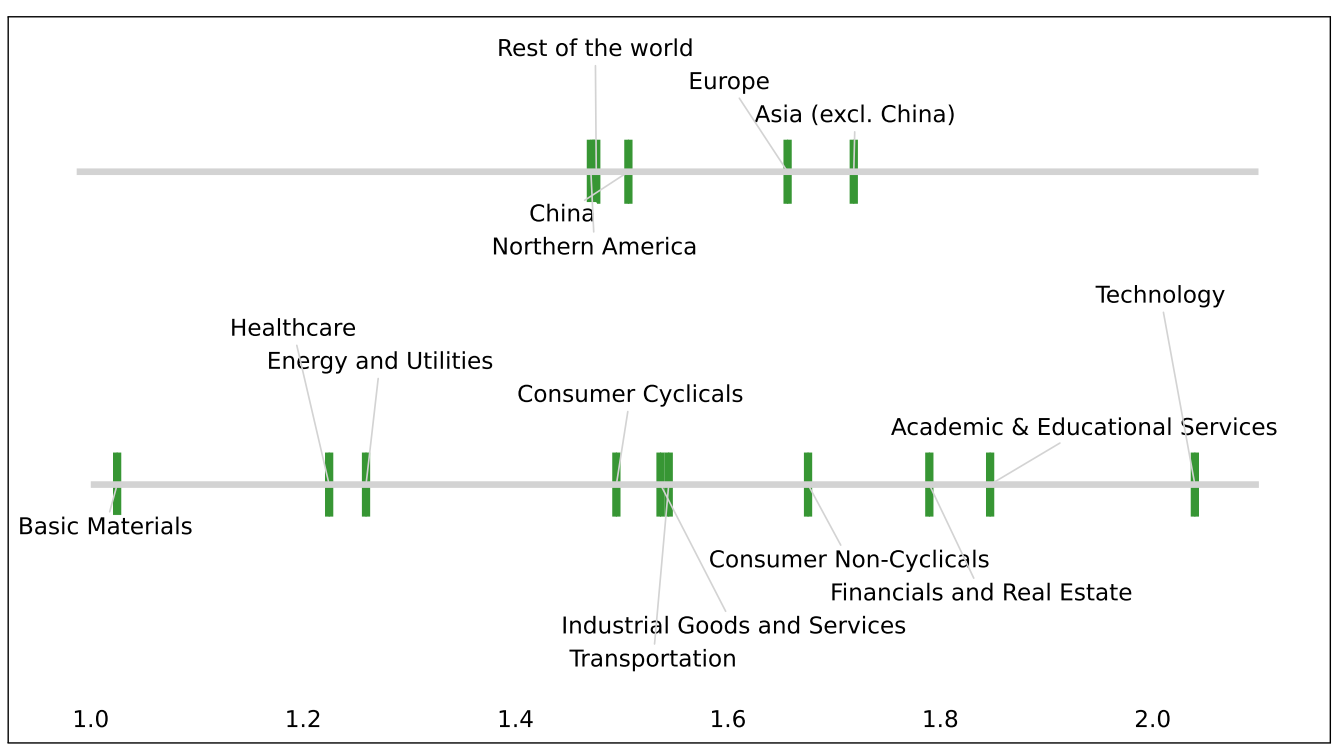

(b) Mean COVID-19 financial/non-financial adjustments by region and sector

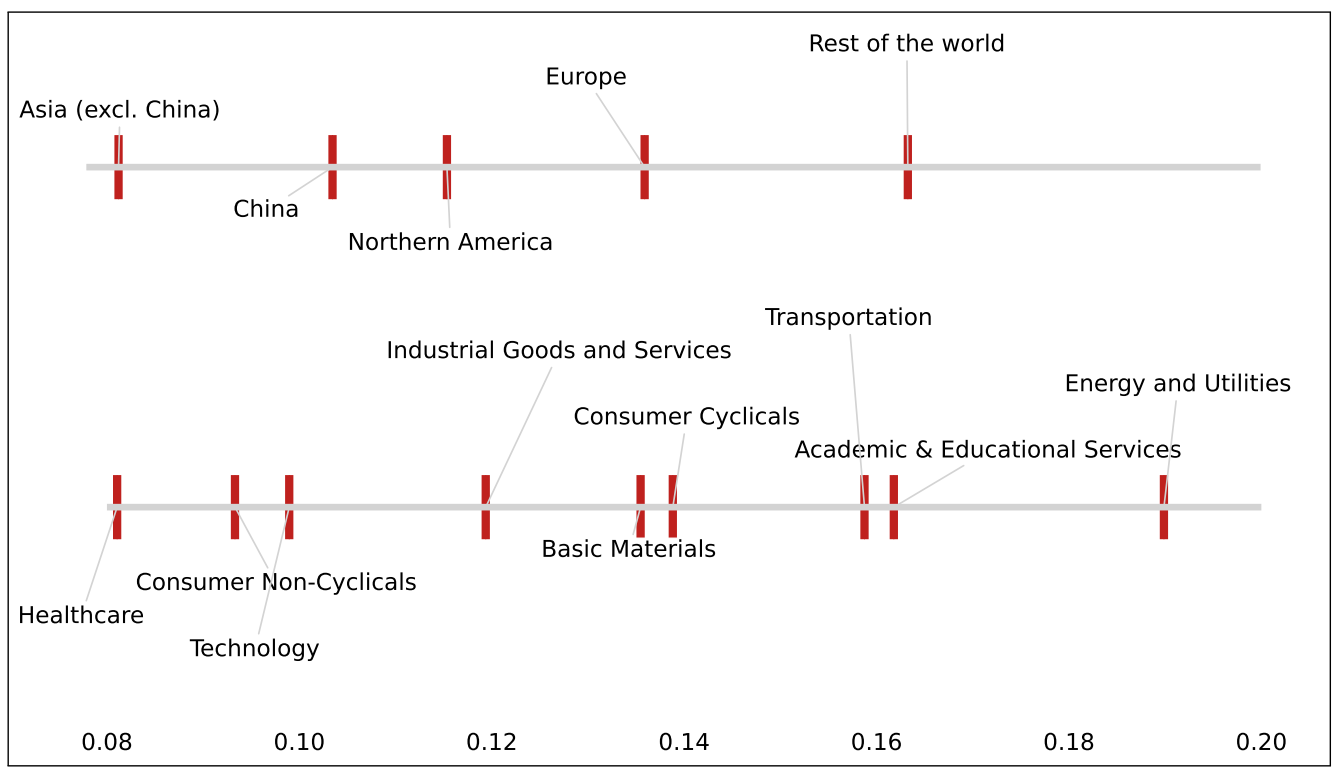

Notes: This figure plots mean COVID-19 demand/supply impacts and COVID-19 financial/non-financial adjustments. COVID-19 demand/supply impacts is the ratio of average COVID-19 demand exposure de $_{i, t}$ to average COVID-19 supply exposure ${ }_{i, t}$, with averages taken over all quarters and firms' headquarter region (top) and firms' sector (bottom), respectively. COVID-19 financial/non-financial adjustments is defined similarly, where COVID19 non-financial adjustments equals the sum of the COVID-19 exposures to the four remaining topics ('supply impacts,' 'cost reductions,' 'government assistance,' and 'demand impacts'). In Panel (b), we exclude firms in the finance and real estate sector. Means are based on earnings conference calls held from January through December 2020. 
Figure 7: Non-financial S\&P 500 firms' COVID-19 demand/supply impacts and COVID-19 financial/non-financial adjustments

(a) COVID-19 demand/supply impacts

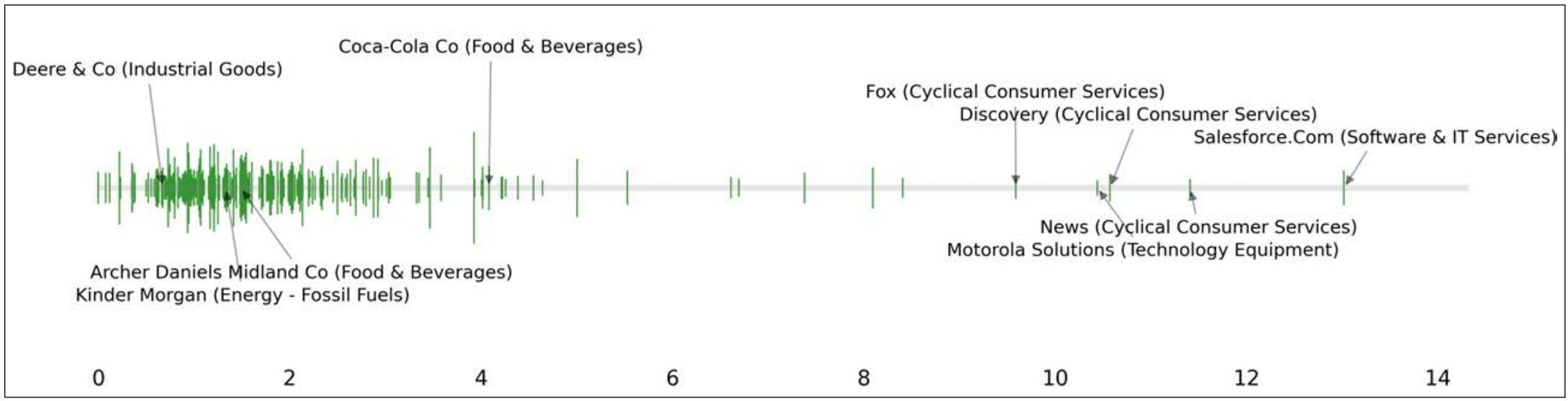

(b) COVID-19 financial/non-financial adjustments

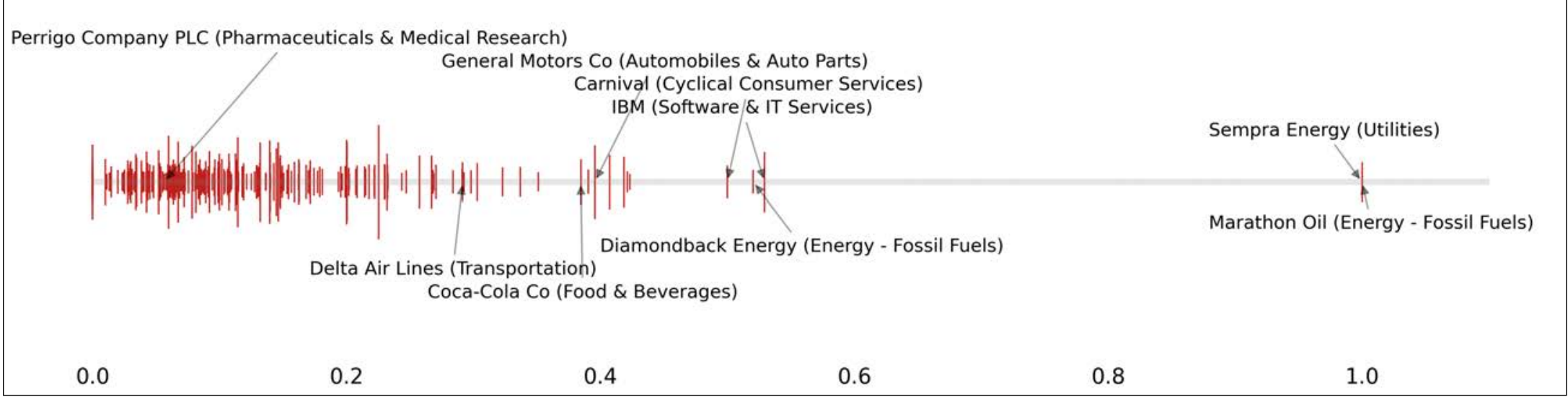

Notes: This figure plots non-financial S\&P 500 firms' COVID-19 demand/supply impacts and COVID-19 financial/non-financial adjustments, based on transcripts of earnings conference calls held from January through December 2020. COVID-19 demand/supply impacts and COVID-19 financial/nonfinancial adjustments are defined as in Figure 6 but with averages taken over all earnings calls from a given firm. Panel (a) excludes Fidelity National Information Services Inc. with COVID-19 demand/supply impacts of 25.3. The size of the marker reflects the firm's size, measured by the latest available total assets. Firms with less than $\$ 10$ billion total assets in 2019 are excluded from the figure. 
Figure 8: Comparison of disease-related topics at the onset of the disease outbreak
COVID-19

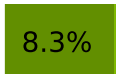
$27.6 \%$
$8.9 \%$
$13.2 \%$
$40.4 \%$
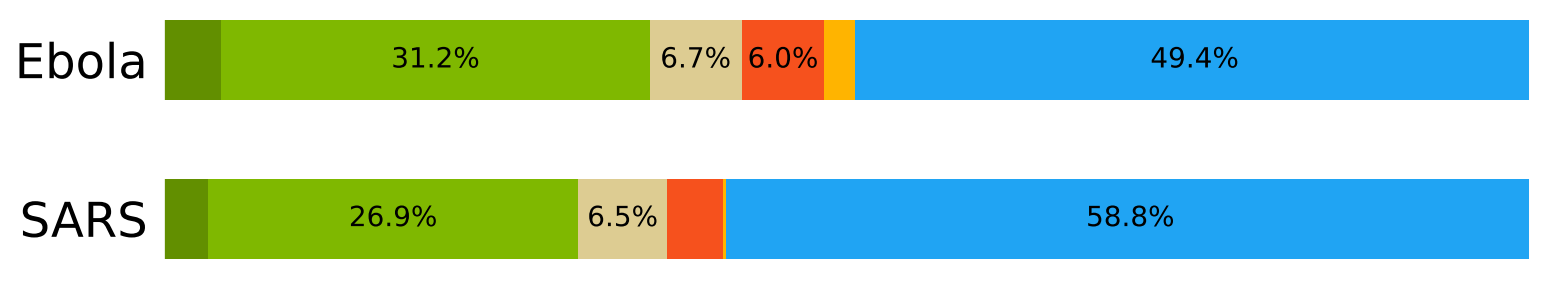
H1N1
$25.0 \%$
$8.2 \% \quad 6.5 \%$
$57.1 \%$
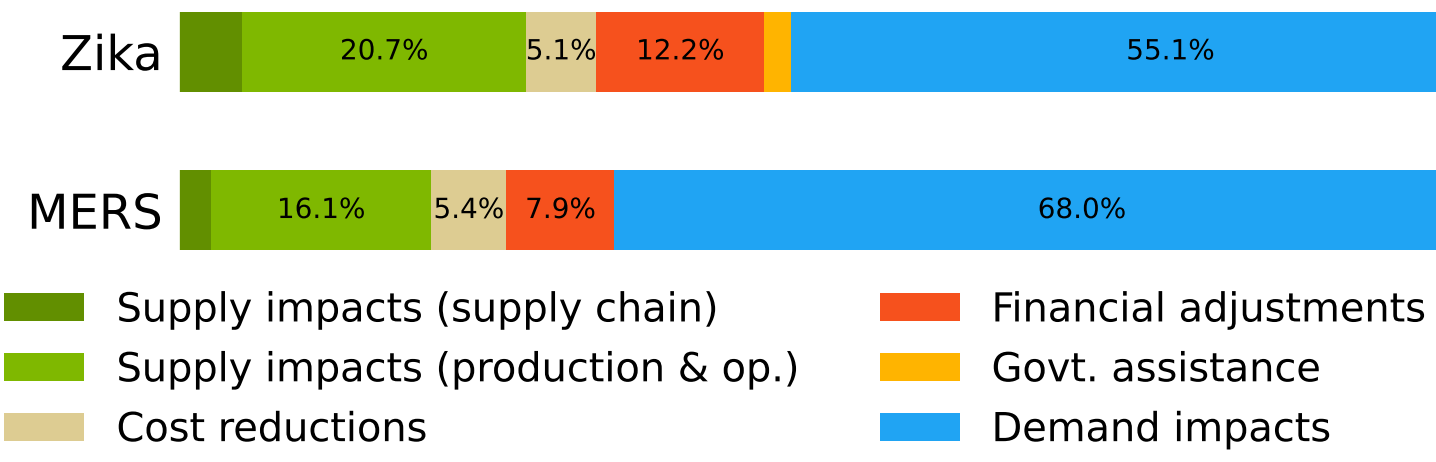

Notes: This figure plots the average share, across all firms, in all disease-related topic ('supply impacts' (i.e., 'supply chain' and 'production and operations'), 'cost reductions,' 'financial adjustments, ' 'government assistance,' 'demand impacts') mentions in the initial three quarters of a disease's outbreak. The initial three quarters are defined as the peak quarter (see Figure 1) plus the immediate quarter before and after. In particular, they are Q4-2019 through Q2-2020 for COVID-19, Q3-2014 through Q1-2015 for Ebola, Q1-2003 through Q3-2003 for SARS, Q1-2009 through Q3-2009 for H1N1, Q4-2015 through Q2-2016 for Zika, and Q2-2015 through Q4-2015 for MERS. A disease-related mention is defined as a sentence triple in which the middle sentence contains a disease-related keyword. 
Figure 9: Average COVID-19 net demand shock ${ }_{i, t}$ and negative supply shock ${ }_{i, t}$ by sector

(a) Average of COVID-19 net demand shock $_{i, t}$

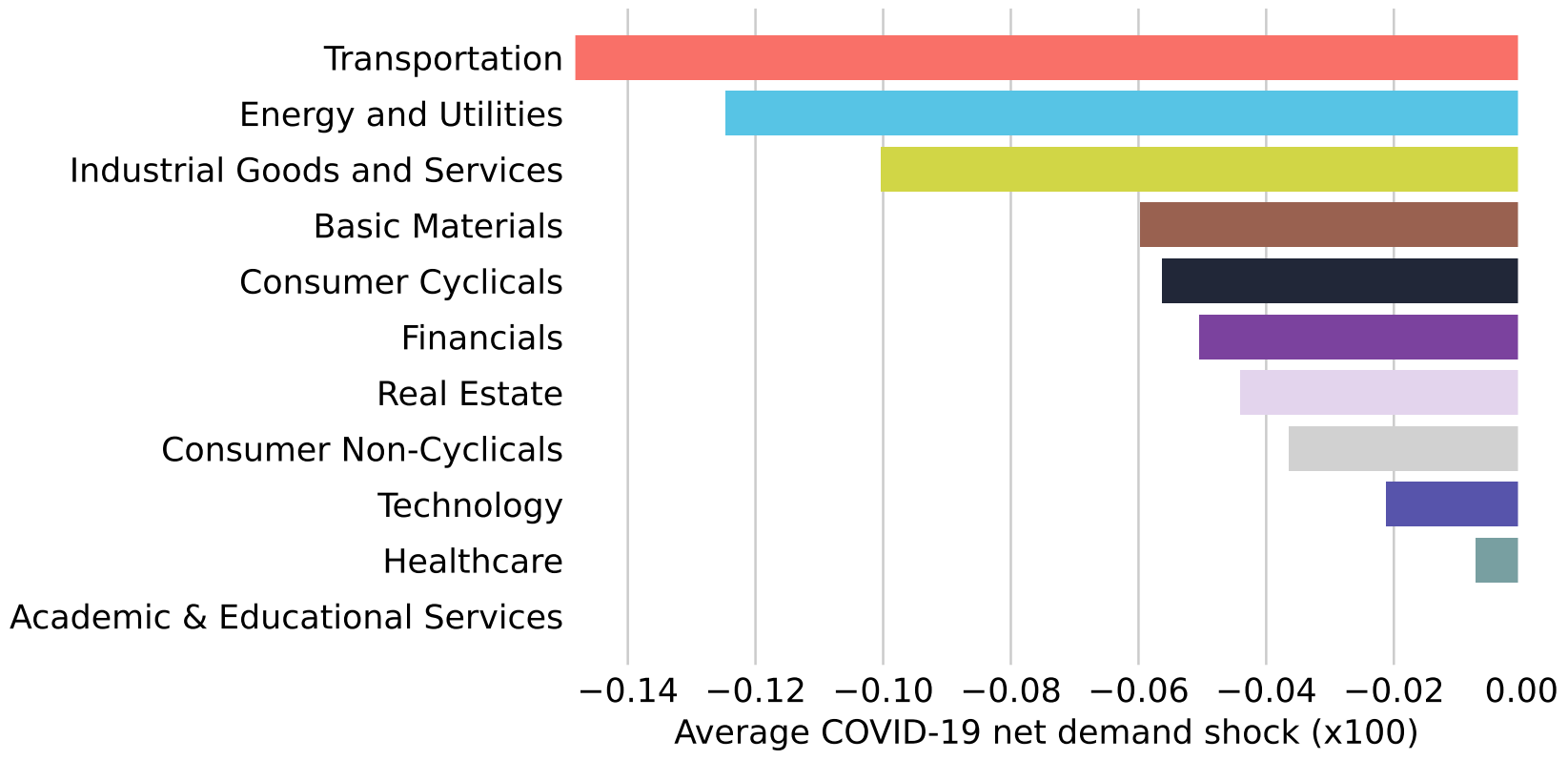

(b) Average of COVID-19 negative supply shock ${ }_{i, t}$

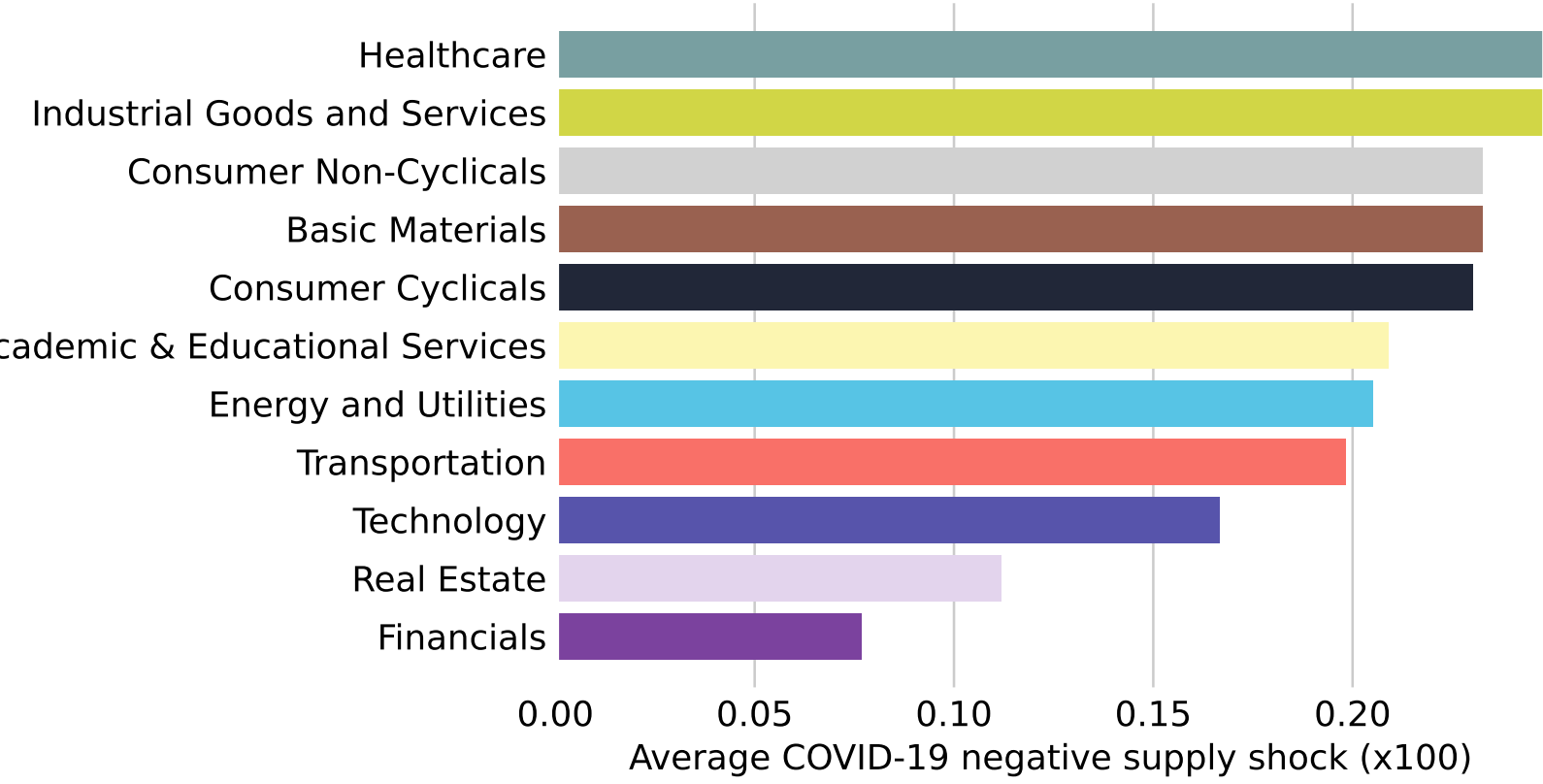

Notes: This figure plots the sector averages of COVID-19 net demand shock $k_{i, t}$ and COVID-19 negative supply shock $_{i, t}$ for all firms with earnings conference calls between January and December 2020. The averages are multiplied by 100 for exposition purposes. The sector classification is based on a firm's "Economic Sector" from the Refinitiv Eikon database. 
Figure 10: Valuation effects from COVID-19 net demand and negative supply shock by sector

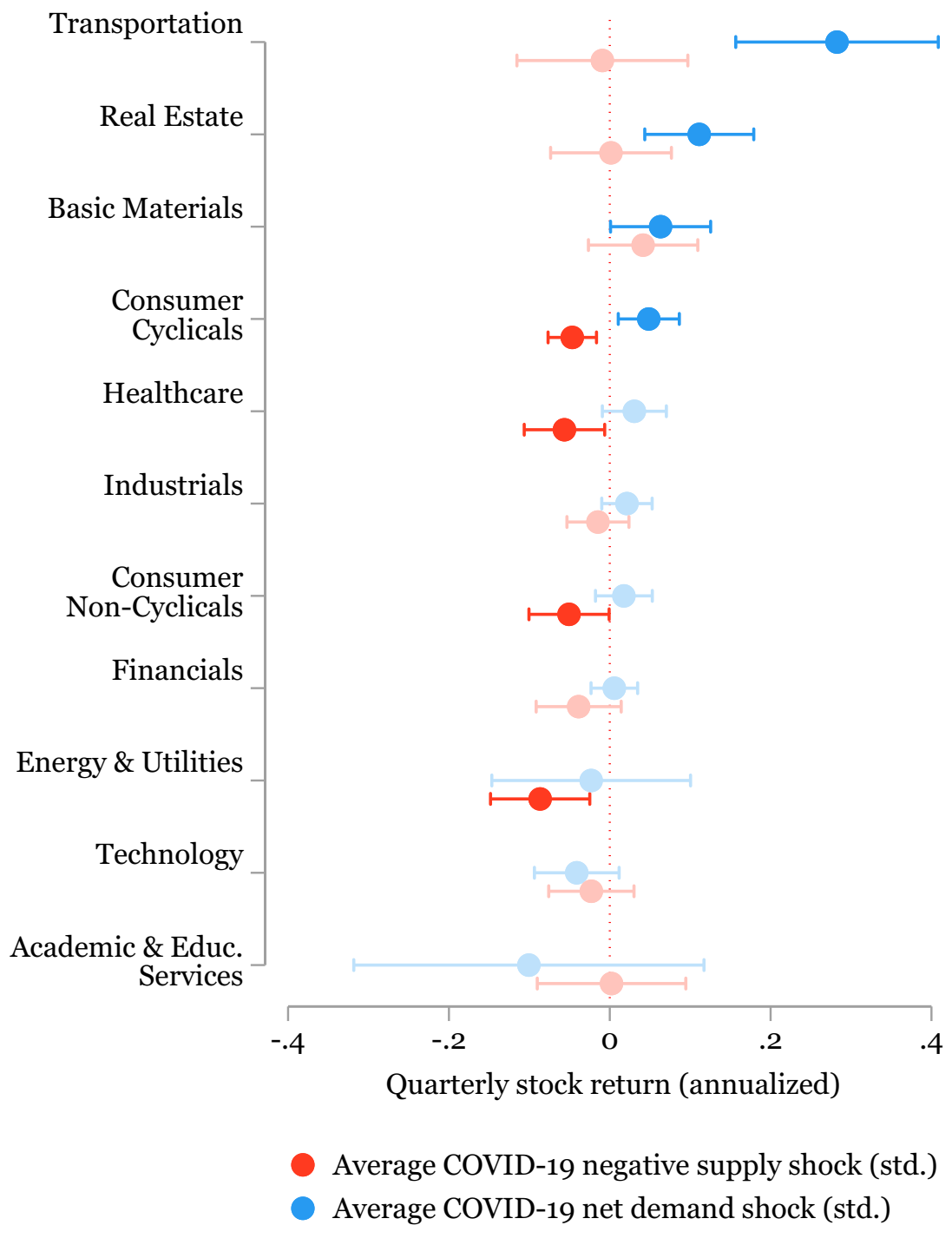


Notes: This figure plots the coefficient estimates and corresponding $95 \%$ confidence intervals for $\beta_{1}$ and $\beta_{2}$ from the following firm-quarter level regression estimated separately for the sectors indicated on the vertical axis:

$$
\begin{aligned}
\text { Return }_{i, t}= & \delta_{s(i)}+\gamma_{t}+\beta_{1} \text { Average COVID-19 net demand shock }(\text { std. })_{i, t} \\
& +\beta_{2} \text { Average COVID-19 negative supply shock }(\text { std. })_{i, t}+\mathbf{x}_{i t}^{\prime} \eta+\varepsilon_{i, t}
\end{aligned}
$$

where Return $_{i, t}$ is the annualized quarterly stock return of firm $i$ during quarter $t ; \delta_{s(i)}$ and $\gamma_{t}$ are sector and quarter fixed effects, respectively; COVID-19 net demand shock and COVID-19 negative supply shock are measured as defined in Section 3 ; and $\mathbf{x}_{i t}$ contains the log of firm $i$ 's total assets in 2019 and the firm's market beta in 2018, both interacted with a time dummy that is equal to one in 2020 and zero otherwise. All other variables are as defined in Table 1 . The sample is restricted to US-based firms and transcripts of these firms' earnings conference calls held in Q1-2020 through Q4-2020. The sector classification is Eikon's Economic sector. Standard errors are clustered at the firm level. The color of coefficients and standard errors that are not statistically significant at the five percent level is muted. 
Table 1: Summary statistics

\begin{tabular}{|c|c|c|c|c|c|c|c|}
\hline & \multicolumn{4}{|c|}{ All firms } & \multicolumn{3}{|c|}{ US firms } \\
\hline & Mean & Median & $\mathrm{SD}$ & $\mathrm{N}$ & Mean & $\mathrm{SD}$ & $\mathrm{N}$ \\
\hline \multicolumn{8}{|l|}{ PANEL A: Firm-QUARTER LEVEL } \\
\hline COVID-19 exposure $_{i, t}$ (std.) & 0.996 & 0.752 & 1.000 & 18,368 & 1.017 & 1.034 & 9,480 \\
\hline COVID-19 sentiment $t_{i, t}$ (std.) & -0.083 & 0.000 & 1.000 & 18,368 & -0.051 & 1.025 & 9,480 \\
\hline COVID-19 positive sentiment $t_{i, t}$ (std.) & 0.526 & 0.000 & 1.000 & 18,368 & 0.589 & 1.086 & 9,480 \\
\hline COVID-19 negative sentiment ${ }_{i, t}$ (std.) & 0.509 & 0.000 & 1.000 & 18,368 & 0.517 & 1.014 & 9,480 \\
\hline COVID-19 risk $k_{i, t}$ (std.) & 0.718 & 0.416 & 1.000 & 18,368 & 0.748 & 1.019 & 9,480 \\
\hline Quarterly stock return (annualized) ${ }_{i, t}$ & -0.004 & 0.049 & 0.399 & 18,368 & 0.011 & 0.434 & 9,480 \\
\hline Stock return $[-1 \text { day, }+1 \text { day }]_{i, t}$ & -0.001 & -0.000 & 0.098 & 18,151 & -0.001 & 0.111 & 9,454 \\
\hline \multicolumn{8}{|l|}{ PANEl B: Firm-Year LEVEl } \\
\hline $\log \left(i_{i, t} / k_{i, t}\right)$ & -2.484 & -2.396 & 0.778 & 8,860 & -2.475 & 0.774 & 4,900 \\
\hline $\log \left(\right.$ employment $\left._{i, t}\right)$ & 1.154 & 1.258 & 1.997 & 7,990 & 0.878 & 2.009 & 4,676 \\
\hline $\log \left(\right.$ revenue $\left._{i, t}\right)$ & 5.564 & 5.705 & 2.077 & 8,454 & 5.365 & 2.161 & 4,551 \\
\hline$S G \mathcal{E} A_{i, t} / \operatorname{asset}_{i, t}$ & 0.052 & 0.034 & 0.055 & 7,783 & 0.062 & 0.060 & 4,232 \\
\hline \multicolumn{8}{|l|}{ PANEL C: FiRM LEVEL } \\
\hline$\overline{\text { COVID-19 net demand shock }}$ (std.) & -0.159 & 0.000 & 1.000 & 4,430 & -0.163 & 0.968 & 2,452 \\
\hline$\overline{\text { COVID-19 negative supply shock }}$ (std.) & 0.566 & 0.126 & 1.000 & 4,430 & 0.618 & 1.041 & 2,452 \\
\hline $\log \left(\right.$ assets in $\left.2019_{i}\right)$ & 7.567 & 7.613 & 2.012 & 4,430 & 7.371 & 2.060 & 2,452 \\
\hline Market beta in $2018_{i}$ & 0.656 & 0.633 & 0.438 & 3,743 & 0.884 & 0.373 & 2,093 \\
\hline
\end{tabular}

Notes: This table shows the mean, median, standard deviation, and the number of observations for the variables used in the regression analysis. Columns 1 to 4 refer to the full sample; and Columns 5 to 7 to the sample of US firms. The unit of the data is firm-quarter, firm-year, and firm level in Panels A, B, and C, respectively. All epidemic disease variables are calculated as defined in Section 2.1 and standardized by their standard deviation in the panel of the main specification. In Panel A, the quarterly sample is restricted to firms for which we have earnings conference calls in 2020. Quarterly stock return (annualized) ${ }_{i, t}$ is the cumulative daily stock return of firm $i$ in quarter $t$, multiplied by 4; Stock return [-1 day,1 day $]_{i, t}$ is the cumulative daily stock return from one day before to one day after the date of the earnings conference call of firm $i$ in quarter $t$. In Panel $\mathrm{B}$, the annual sample is restricted to firms for which we have earnings conference calls in 2019 and 2020. $\log \left(i_{i, t} / k_{i, t}\right)$ is the annual average of the $\log$ of winsorized (at the first and last percentile) quarterly investment rate (calculated using the perpetual inventory method as in, for example, Stein and Stone (2013)) of firm $i$ and year $t ; \log \left(\right.$ employment $\left._{i, t}\right)$ is the $\log$ of winsorized (at the first and last percentile) annual employment (emp in Compustat) of firm $i$ and year $t ; \log \left(\right.$ revenue $\left._{i, t}\right)$ is the annual average of the $\log$ of winsorized (at the first and last percentile) quarterly revenue (revtq in Compustat) of firm $i$ and year $t$; and $S G \mathscr{G} A_{i, t} /$ assets $_{i, t}$ is the annual average of the winsorized (at the first and last percentile) quarterly selling, general \& administrative expenses (xsgaq in Compustat) divided by assets (atq in Compustat) of firm $i$ and year $t$. In Panel $\mathrm{C}, \log ($ assets in 2019 $)$ is the annual average of the $\log$ of quarterly assets of firm $i$ in 2019; Market beta in 2018 is firm $i$ 's market beta in 2018, obtained by regressing firm $i$ 's daily stock returns in 2018 on the contemporaneous daily S\&P 500 index. 
Table 2: Correlation COVID-19 exposure $e_{i, t}$, sentiment $t_{i, t}$, and risk $_{i, t}$ with stock returns

\begin{tabular}{|c|c|c|c|c|c|c|}
\hline \multirow{3}{*}{ Panel A: Firm-Quarter Q1-Q4 2020} & \multicolumn{3}{|c|}{ All firms } & \multicolumn{3}{|c|}{ US-based firms } \\
\hline & \multicolumn{6}{|c|}{ Annualized quarterly stock return $_{i, t}$} \\
\hline & $(1)$ & $(2)$ & $(3)$ & $(4)$ & $(5)$ & $(6)$ \\
\hline COVID-19 exposure $_{i, t}$ (std.) & $\begin{array}{c}-0.089^{* * *} \\
(0.010)\end{array}$ & & & $\begin{array}{c}-0.120^{* * *} \\
(0.017)\end{array}$ & & \\
\hline COVID-19 sentiment $i_{i, t}$ (std.) & & $\begin{array}{c}0.058^{* * *} \\
(0.009)\end{array}$ & & & $\begin{array}{c}0.055^{* * *} \\
(0.014)\end{array}$ & \\
\hline COVID-19 positive sentiment $t_{i, t}$ (std.) & & & $\begin{array}{c}0.003 \\
(0.011)\end{array}$ & & & $\begin{array}{l}-0.013 \\
(0.016)\end{array}$ \\
\hline COVID-19 negative sentiment ${ }_{i, t}$ (std.) & & & $\begin{array}{c}-0.059^{* * *} \\
(0.011)\end{array}$ & & & $\begin{array}{c}-0.076^{* * *} \\
(0.018)\end{array}$ \\
\hline COVID-19 risk rit $_{\text {(std.) }}$ & & $\begin{array}{c}-0.051^{* * *} \\
(0.009)\end{array}$ & $\begin{array}{c}-0.038^{* * *} \\
(0.011)\end{array}$ & & $\begin{array}{c}-0.074^{* * *} \\
(0.015)\end{array}$ & $\begin{array}{c}-0.051^{* * *} \\
(0.017)\end{array}$ \\
\hline$R^{2}$ & 0.498 & 0.498 & 0.498 & 0.506 & 0.505 & 0.506 \\
\hline$N$ & 18,368 & 18,368 & 18,368 & 9,480 & 9,480 & 9,480 \\
\hline \multirow[t]{2}{*}{ Panel B: Around earnings Call } & \multicolumn{6}{|c|}{ Stock return $[-1 \text { day, }+1 \text { day }]_{i, t}$} \\
\hline & $(1)$ & $(2)$ & $(3)$ & (4) & $(5)$ & $(6)$ \\
\hline COVID-19 exposure est $_{\text {(std.) }}$ & $\begin{array}{c}-0.003^{* * *} \\
(0.001)\end{array}$ & & & $\begin{array}{c}-0.005^{* * *} \\
(0.001)\end{array}$ & & \\
\hline COVID-19 sentiment $t_{i, t}$ (std.) & & $\begin{array}{c}0.005^{* * *} \\
(0.001)\end{array}$ & & & $\begin{array}{c}0.006^{* * *} \\
(0.001)\end{array}$ & \\
\hline COVID-19 positive sentiment $t_{i, t}$ (std.) & & & $\begin{array}{c}0.001 \\
(0.001)\end{array}$ & & & $\begin{array}{c}0.000 \\
(0.002)\end{array}$ \\
\hline COVID-19 negative sentiment $t_{i, t}$ (std.) & & & $\begin{array}{c}-0.005^{* * *} \\
(0.001)\end{array}$ & & & $\begin{array}{c}-0.007^{* * *} \\
(0.002)\end{array}$ \\
\hline COVID-19 risk $k_{i, t}$ (std.) & & $\begin{array}{l}-0.001 \\
(0.001)\end{array}$ & $\begin{array}{l}-0.001 \\
(0.001)\end{array}$ & & $\begin{array}{l}-0.001 \\
(0.002)\end{array}$ & $\begin{array}{c}0.001 \\
(0.002)\end{array}$ \\
\hline$R^{2}$ & 0.024 & 0.026 & 0.026 & 0.029 & 0.031 & 0.031 \\
\hline$N$ & 18,159 & 18,159 & 18,159 & 9,456 & 9,456 & 9,456 \\
\hline Quarter FE & yes & yes & yes & yes & yes & yes \\
\hline Sector FE & yes & yes & yes & yes & yes & yes \\
\hline
\end{tabular}

Notes: This table reports, in Panel A, regression estimates at the firm-quarter level (i.e., annualized Q1-Q4 2020 stock returns) and, in Panel B, at the firm-earnings call level (i.e., [ -1 day, +1 day] stock return around the date of firm $i$ 's earnings conference call in quarter t). All regressions control for firm i's log of total assets in 2019 and the firm's market beta in 2018. Sector fixed effects are defined using Refinitiv Eikon's Business sector, which has 30 sectors in our sample. Standard errors are clustered at the firm level. ***, **, and * denote statistical significance at the 1,5 , and $10 \%$ level, respectively. 
Table 3: Word patterns for each disease-related topic

\section{A sentence triple conforms to a given topic ...}

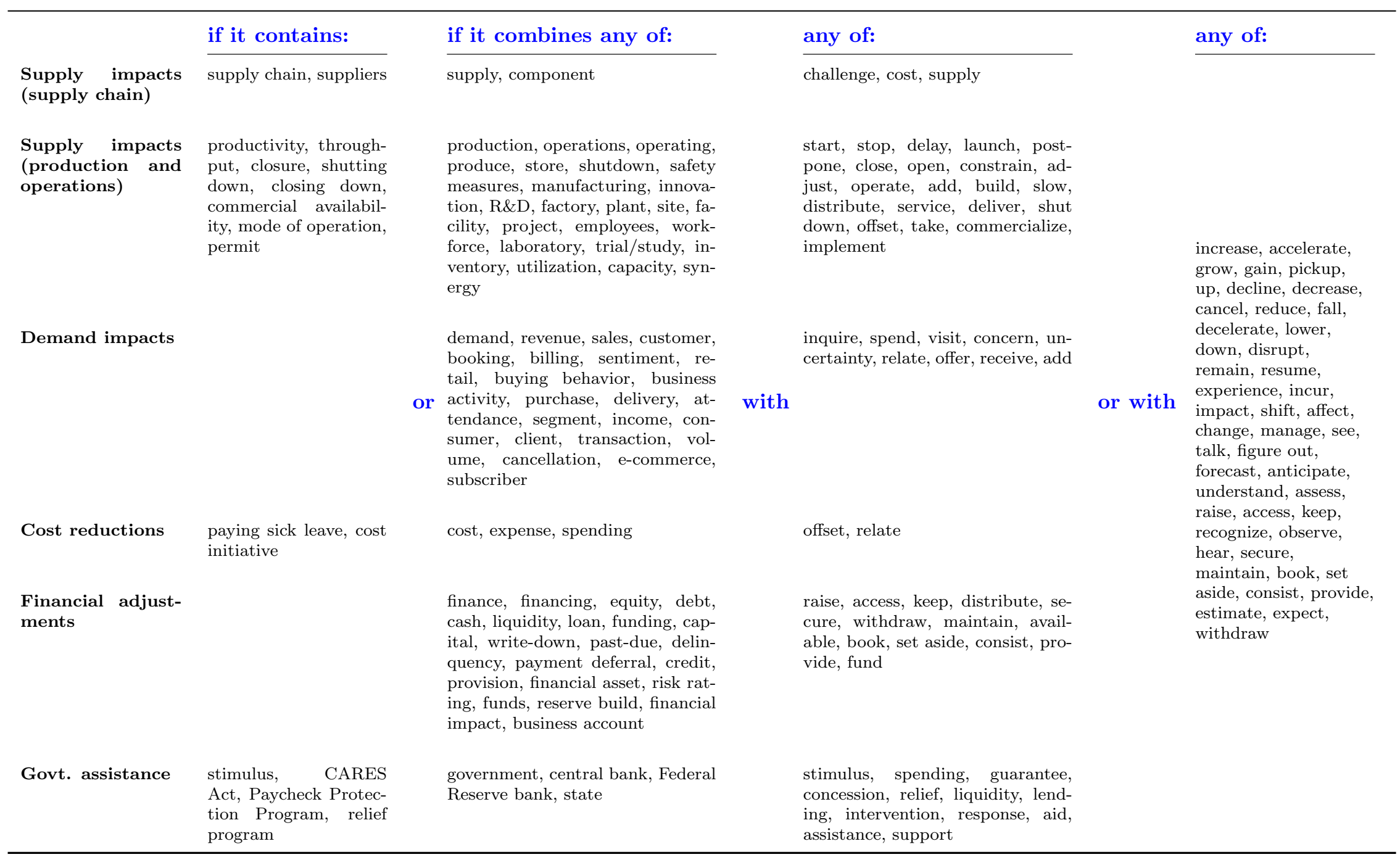

Notes: This table lists the word patterns used in classifying each of the disease-related topics: 'supply chain,' 'production and operations,' 'cost,' 'demand,' 'finance,' 'government.' Verbs are stemmed prior to matching (e.g., "increase" becomes "increas"; which allows for a match with "increase," "increasing," "increased," etc). Nouns allow for singular and plural. Word combinations are required to be found in close vicinity to each other (i.e., within 100 characters). In addition, each topic may impose specific restrictions on words that occur between a word pair. These specific restrictions are listed in Appendix Table 9. 
Table 4: 'Financial adjustments,' 'cost reductions,' and 'government assistance' snippets

\section{Financial adjustments}

- "Thanks, El. I am pleased to present a condensed summary of the first quarter results for Cineplex Inc. and to provide additional detail on the financial impacts of COVID-19 on our operations. I would like to start with the $\$ 173.1$ million impairment charge we recorded in Q1." (Cineplex Inc; 30-Jun-2020)

- "And for foreign currency loan, except for China, most of the cities where our overseas branches and subsidiaries are located are having lockdown, which resulted in the flattish momentum of loan growth in first quarter. So we anticipate there'll be low single-digit growth for foreign currency loan because we don't know how long this COVID-19 will last." (CTBC Financial Holding Co Ltd; 11-May-2020)

- "So turning now to cash in more detail on Slide 10. The first half had been strong, and we were well set up for the second and then COVID struck. And first, the free cash flow, which has also been impacted by COVID." (WH Smith PLC; 12-Nov-2020)

\section{Cost reductions}

- "The carryover of last year's price investment and the temporary closures of our auto care centers and vision centers negatively affected the margin rate. The approximate $\$ 1.2$ billion of incremental COVIDrelated costs as well as the restructuring costs negatively affected expense leverage by about 170 basis points. As a result, the U.S. segment deleveraged 41 basis points." (Walmart Inc; 18-Aug-2020)

- "While most of these decisions were difficult for us to make, we made them with an eye towards not placing at risk our longer-term growth priorities and our competitive position. In late March, we made the difficult decision to resize our store and store support center payroll costs in anticipation that sales could continue to decline for some period of time from the COVID-19 pandemic and that a recovery could be slow once the economy begins to reopen." (Floor \& Decor Holdings Inc; 30-April-2020)

- "Changes have also been made to lapse in claims assumptions to allow for price increases, increased claims and reinsurance costs and potential impacts from COVID-19. To be more specific, we have allowed for COVID-19 shorter-term impacts for the next 2 years to both our best estimate assumptions and embedded value calculations for both claims and lapses." (Clearview Wealth Ltd; 25-August-2020)

\section{Government assistance}

- "The bank's results in Q3 were negatively impacted by a full quarter of COVID-19, which resulted in higher loan loss provisions and lower customer activity. Lower delinquency resulted from the impact of the government stimulus and the bank payment deferral programs, while lower consumer spending also contributed to the lower revolving credit utilization rates." (Bank of Nova Scotia; 25-August-2020)

- "When we applied for the Small Business Administration's Payroll Protection Program (sic) [Paycheck Protection Program], we were uncertain of our ability to maintain our sales growth due to the restrictions on elective procedures. We were uncertain if we could actually help with the treatment of COVID patients in a meaningful way. We were uncertain if we were able to continue to raise money from the equity markets." (CHF Solutions Inc; 12-May-2020)

- "Pinnacle continues its approach of a well-balanced and granular portfolio. While our second quarter credit metrics likely don't yet evidence the full impact of COVID on our loan portfolio, we understand that its fiscal stimulus and PPP proceeds are expanded. Absent further stimulus, we may see these credit metrics change." (Pinnacle Financial Partners Inc; 22-July-2020)

Notes: This table shows three annotated examples of sentence triples (i.e., three consecutive sentences, if available, by the same speaker with the middle sentence containing a COVID-19-related keyword) about the 'financial adjustments,' 'cost reductions,' and 'government assistance' topic, respectively. The word pattern match (described in Table 3) that identifies the topic is highlighted in blue. 
Table 5: Comparison with alternative text classifiers

\begin{tabular}{lrcc}
\hline & Precision & Recall & F1 score \\
\hline Pattern-based classifier & 0.72 & $\mathbf{0 . 6 8}$ & $\mathbf{0 . 6 9}$ \\
Naive Bayes & 0.53 & 0.46 & 0.47 \\
Logistic Regression & $\mathbf{0 . 7 6}$ & 0.45 & 0.47 \\
Feedforward Neural Network & 0.45 & 0.53 & 0.46 \\
\hline
\end{tabular}

Notes: This table reports the precision, recall, and F1 score of different classifiers: pattern-based classifier, Naive Bayes, Logistic Regression, and Feedforward Neural Network. The highest score per column is highlighted in bold. The three metrics - precision, recall, and F1 score - are the average of each classifier's respective scores across the four individual topic categories: 'demand,' 'production and operations,' 'supply chain,' and 'remainder.' (Note: a sentence triple can be classified to more than one topic category.) The reported scores are based on evaluating the method's predictions on the test set of 250 sentences; for Naive Bayes, Logistic Regression, and Feedforward Neural Network, the model's hyperparameters were selected as having the best average F1 score across a 5-fold cross-validation of the training dataset, with each fold running a grid search of all possible hyperparameters specified in Appendix Table 11. For Naive Bayes, Logistic Regression, and Feedforward Neural Network, we use the bag-of-words representation of the sentence triples as the unit of analysis. We remove stopwords and use the remaining unigrams, resulting in a vocabulary of 3,871 unigrams. For Feedforward Neural Network we additionally de-mean and standardize the data. 
Table 6: 'Negative supply,' 'negative demand,' and 'positive demand shock' snippets

\section{Negative supply shock}

- "On the top line, organic sales in the first quarter declined by $1.3 \%$, including the negative impact of our facilities in China being closed for a full month due to the COVID-19 pandemic." (RR Donnelley \& Sons Co; 29-Apr-2020)

- "We also experienced delayed deliveries from our suppliers due to COVID-related factory shutdowns and piece part supply chain interruptions." (Westell Technologies Inc; 18-Jun-2020)

- "We've been facing countrywide lockdowns in Italy and Spain and voluntary production stoppages at virtually all automotive manufacturers in the region, resulting in significant reductions in production capacity at all automotive suppliers." (KEMET Corp; 14-May-2020)

- "Installation [of the offshore loading buoy] was delayed due to COVID-19 concerns on operations earlier this year." (Tullow Oil PLC; 09-Sep-2020)

\section{Negative demand shock}

- "The old business outlook seems to be tough due to major decline in fuel demand because of restriction of movement due to COVID-19, and KBC suffered the biggest impact." (Yokogawa Electric Corp; 12-May2020)

- "Turning now to the actions we're taking in the face of COVID-19 and the resulting severe disruption to global demand for air travel." (American Airlines Group Inc; 23-Jul-2020)

- "However, in Q2, as COVID led - COVID-19 led to schools' closures and exams being canceled, our revenues started to be impacted, largely driven by a significant downturn in order intake in our Resources business." (RM PLC; 07-Jul-2020)

- "The decline in net revenue was primarily due to the impact of COVID-19." (DASAN Zhone Solutions Inc; 7-May-2020)

\section{Positive demand shock}

- "Revenue growth was driven in part by the sustained rapid increase in the number of biologics in development as well as new opportunities such as cell and gene therapies and COVID-19 therapeutics that continue to propel market growth." (Charles River Laboratories International Inc; 05-Aug-2020)

- "As coronavirus hit the U.S. this spring, Tower saw a spike in website visits and customer service calls, creating both an opportunity and a pain point." (Yext Inc; 03-Sep-2020)

- "Again, driven by underlying - good underlying pharmaceutical market growth and then also the COVID pandemic-related demand increase." (Oriola Oyj; 24-Apr-2020)

- "In the first quarter, EVO delivered $4 \%$ normalized revenue growth and 12\% normalized adjusted EBITDA growth, which reflects the company's strong performance in January and February, offset by the impact of COVID-19 beginning in early to mid-March." (EVO Payments Inc; 8-May-2020)

Notes: This table shows annotated examples of sentences that identify demand and supply shocks. Specifically, for each shock (i.e., 'negative supply,' 'negative demand,' and 'positive demand') we list four example sentences from sentence triples. The word pattern match for each shock is highlighted in blue; positive and negative sentiment words in green and red, respectively. 
Table 7: Correlation COVID-19 net demand and negative supply shock with stock returns

\begin{tabular}{|c|c|c|c|}
\hline & \multirow{2}{*}{ All firms } & \multicolumn{2}{|c|}{ US firms } \\
\hline & & All & Large \\
\hline & \multicolumn{3}{|c|}{ Annualized quarterly stock return $_{i,}$} \\
\hline & (1) & $(2)$ & $(3)$ \\
\hline COVID-19 net demand shock $_{i, t}$ (std.) & $\begin{array}{c}0.028 * * * \\
(0.006)\end{array}$ & $\begin{array}{c}0.020^{*} \\
(0.011)\end{array}$ & $\begin{array}{c}0.031^{* *} \\
(0.013)\end{array}$ \\
\hline COVID-19 negative supply shock ${ }_{i, t}$ (std.) & $\begin{array}{c}-0.013^{* *} \\
(0.006)\end{array}$ & $\begin{array}{c}-0.033^{* * *} \\
(0.009)\end{array}$ & $\begin{array}{c}-0.023^{* *} \\
(0.009)\end{array}$ \\
\hline$R^{2}$ & 0.497 & 0.504 & 0.541 \\
\hline$N$ & 18,368 & 9,480 & 6,823 \\
\hline Quarter FE & yes & yes & yes \\
\hline Sector FE & yes & yes & yes \\
\hline
\end{tabular}

Notes: This table reports the coefficient estimates and standard errors for $\beta_{1}$ and $\beta_{2}$ from the following regression:

$$
\begin{aligned}
\text { Return }_{i, t}= & \delta_{s(i)}+\gamma_{t}+\beta_{1} \text { Average COVID-19 net demand shock }(\text { std. })_{i, t} \\
& +\beta_{2} \text { Average COVID-19 negative supply shock }(\text { std. })_{i, t}+\mathbf{x}_{i t}^{\prime} \eta+\varepsilon_{i, t}
\end{aligned}
$$

where Return $n_{i, t}$ is the annualized quarterly stock return of firm $i$ during quarter $t ; \delta_{s(i)}$ and $\gamma_{t}$ are sector and quarter fixed effects, respectively; COVID-19 net demand shock and COVID-19 negative supply shock are measured as defined in Section 3 ; and $\mathbf{x}_{i t}$ contains the log of firm $i$ 's total assets in 2019 and the firm's market beta in 2018, both interacted with a time dummy. All other variables are as defined in Table 1. The sample is restricted to earnings conference calls held between Q1-2020 and Q4-2020. Large US firms are firms with more than 500 employees (based on the emp variable from Compustat). Sector fixed effects are defined using Refinitiv Eikon's Business sector, which has 30 sectors in our sample. Standard errors are clustered by firm. ${ }^{*}, * *$, and $* * *$ indicate statistical significance at the 10,5 , and 1 percent significance, respectively. 
Table 8: Validation of COVID-19 demand and negative supply shocks

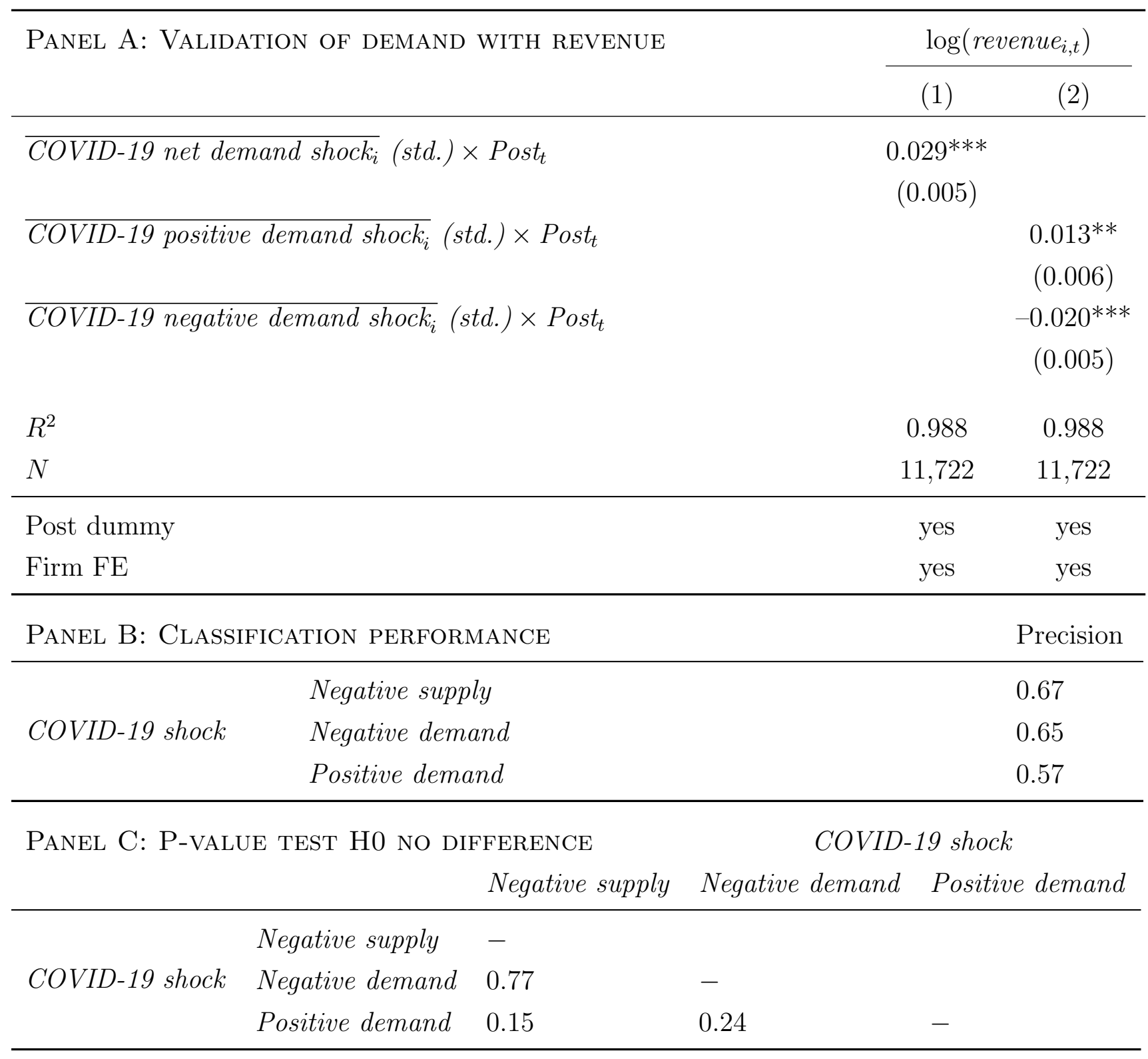

Notes: This table reports results from two types of validation exercises of the COVID-19 demand and supply shocks. Panel A shows the estimated coefficients and standard errors from the difference-in-differences regression as specified in equation (4). The unit of the regression is a firm-year pair, and there are two years (2019 and 2020). The outcome variable is the $\log$ of the winsorized (at the first and last percentile) in average quarterly revenue (revtq in Compustat) in the respective year. Post $t$ is a binary variable equal to one in 2020 and zero in 2019. Consistent with equation (4), all regressions control for Post ${ }_{t}$ and the interaction of the log of firm $i$ 's total assets in 2019 and Post . All other variables are defined in Table 1. Standard errors are clustered by firm. *, **, and $* * *$ indicate statistical significance at the 10,5 , and 1 percent significance, respectively. Panel B shows the precision of the pattern-based classifier for three measures: COVID-19 negative supply shock, COVID-19 negative demand shock, and COVID-19 positive demand shock. The numbers in the 'precision' column are calculated based on a test set of 100 sentence triples, created separately for each of the three measures. Each test set is created in two steps. First, we randomly draw 100 sentence triples from the set of sentence triples that the pattern-based classifier classifies as true positives for the respective category. Second, we task a human reader to manually classify each sentence triple as to whether it conforms to both the sentiment and the topic. Precision is defined as the number of true positives divided by the sum of true and false positives. Panel $\mathrm{C}$ shows the $p$-value from testing pairwise differences of the precision in Panel $\mathrm{B}_{0}$ (assuming a normal distribution). 
Table 9: Correlation COVID-19 net demand \& negative supply shock with firm outcomes

\begin{tabular}{|c|c|c|c|}
\hline \multicolumn{4}{|c|}{ PANEL A: InVESTMENT } \\
\hline & \multirow{2}{*}{ All firms } & \multicolumn{2}{|c|}{ US firms } \\
\hline & & All & Large \\
\hline & \multicolumn{3}{|c|}{$\log \left(i_{i, t} / k_{i, t}\right)$} \\
\hline & $(1)$ & $(2)$ & $(3)$ \\
\hline$\overline{\text { COVID-19 net demand shock }}$ (std.) $\times$ Post $_{t}$ & $\begin{array}{c}0.037^{* * *} \\
(0.013)\end{array}$ & $\begin{array}{c}0.056^{* * *} \\
(0.020)\end{array}$ & $\begin{array}{c}0.033^{* *} \\
(0.016)\end{array}$ \\
\hline$\overline{\text { COVID-19 negative supply shock }}$ (std.) $\times$ Post $_{t}$ & $\begin{array}{l}-0.004 \\
(0.015)\end{array}$ & $\begin{array}{c}0.003 \\
(0.022)\end{array}$ & $\begin{array}{l}-0.021 \\
(0.020)\end{array}$ \\
\hline$R^{2}$ & 0.758 & 0.739 & 0.788 \\
\hline$N$ & 8,860 & 4,896 & 3,720 \\
\hline \multicolumn{4}{|c|}{ PANEL B: EMPLOYMENT } \\
\hline & \multicolumn{3}{|c|}{$\log \left(\right.$ employees $\left._{i, t}\right)$} \\
\hline & $(1)$ & $(2)$ & $(3)$ \\
\hline$\overline{\text { COVID-19 net demand shock }}$ (std.) $\times$ Post $_{t}$ & $\begin{array}{c}0.008 \\
(0.008)\end{array}$ & $\begin{array}{c}0.007 \\
(0.016)\end{array}$ & $\begin{array}{c}0.019^{* * *} \\
(0.006)\end{array}$ \\
\hline$\overline{\text { COVID-19 negative supply shock }}($ std. $) \times$ Post $_{t}$ & $\begin{array}{c}-0.010^{*} \\
(0.005)\end{array}$ & $\begin{array}{l}-0.012 \\
(0.008)\end{array}$ & $\begin{array}{c}-0.010^{* *} \\
(0.005)\end{array}$ \\
\hline$R^{2}$ & 0.996 & 0.995 & 0.993 \\
\hline$N$ & 10,302 & 5,268 & 3,918 \\
\hline Post dummy & yes & yes & yes \\
\hline Firm FE & yes & yes & yes \\
\hline
\end{tabular}

Notes: This table reports the estimated coefficients and standard errors from the difference-indifferences regression specified in equation (4). The unit of the regression is a firm-year pair, and there are two years (2019 and 2020). In Panel A, $i_{i, t} / k_{i, t}$ is the average investment rate of firm $i$ in year $t$, calculated according to the perpetual inventory method detailed in Stein and Stone (2013). In Panel B, employment $i$ is the number of employees in thousands (emp in Compustat). Both outcome variables are winsorized at the first and last percentile prior to taking the log. Post is a binary variable equal to one in 2020 and zero in 2019. Consistent with equation (4), all regressions

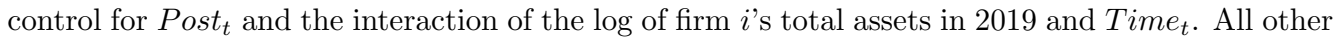
variables are defined in Table 1. Large US firms are firms with more than 500 employees (based on the emp variable from Compustat). Standard errors are clustered by firm. *, **, and *** indicate statistical significance at the 10,5 , and 1 percent significance, respectively. 


\title{
Appendix \\ "Firm-Level Exposure to Epidemic Diseases: COVID-19, SARS, and H1N1"
}

\author{
Tarek A. Hassan, Stephan Hollander, Laurence van Lent, \\ Markus Schwedeler, and Ahmed Tahoun
}

We organize this appendix as follows. In Appendix A, we discuss how our (supervised) approach compares to a frequently used unsupervised topic identification approach: LDA. In Appendix B, we provide several additional figures and tables that we refer to in the main text of the paper.

\section{A. IDENTIFYING TOPICS: DETAILS AND DISCUSSION}

This appendix contains a brief comparison of our topic discovery step with a popular unsupervised method for topic discovery: Latent Dirichlet Allocation (LDA). Specifically, we compare the five categories from our topic discovery step with LDA. Recall that we leverage our team members' economic judgment to find a set of categories that does justice to our goal of tracing the channels of a particular macro-level shock - the outbreak of COVID-19-to a firm. Thus, the categories should strike a reasonable balance between representing multifaceted coronavirus-related discussions and being economically meaningful for subsequent analyses. It is worth noting that this balance is difficult to strike even for a human reader. We report the results from several LDA runs in the form of word clouds in Appendix Figure 3. It is not always easy to make sense of word clouds. Nevertheless, the word clouds suggest that none of the topics align with what economists would view as supply or demandrelated impacts, let alone represent useful categories that allow us to trace the channels of the coronavirus outbreak to firms.

We believe that it is not accidental but results from a key logical issue that limits the applicability of automated topic detection in this context. Conversations in earnings calls are multi-dimensional by nature and 'off-the-shelf' LDA algorithms cannot tell in which of these dimensions we are interested. For example, a typical discussion of a supply-side impact might read, "On the top line, organic sales in the first quarter declined by $1.3 \%$, including the 
negative impact of our facilities in China being closed for a full month due to the COVID-19 pandemic." This discussion touches on multiple logical planes: the firm's total profits, closed facilities, supply-side impacts, and the firm's activities in China. LDA attempts to cluster topics discussed and, instead of identifying the relation between this firm's closed facilities and another firm's difficulties in sourcing parts, might find that this discussion is closest to other discussions of the firms' foreign activities, profits, or closed facilities. All of these inferences are, of course, correct. Deciding on which of these dimensions we are interested in is thus inherently a task that requires judgment, which we exert by defining topics in our training sample.

In addition, it is worth pointing out that researchers have significant discretion when using LDA to identify a text's topics, as the algorithm requires the choice of several parameters that can meaningfully change the resulting topics. With LDA's non-deterministic nature (multiple runs on the same data and using the same parameters may generate different topics), this suggests that it is not trivial to credibly tie the researcher's hands and generate a reproducible result with LDA. Taken together, we believe that for our context the usage of our economic judgment, as opposed to unsupervised methods such as LDA, is appropriate. 


\section{B. AdDitional Figures AND tables}

Appendix Figure 1: Percentage of earnings calls discussing epidemic diseases across regions

(a) China

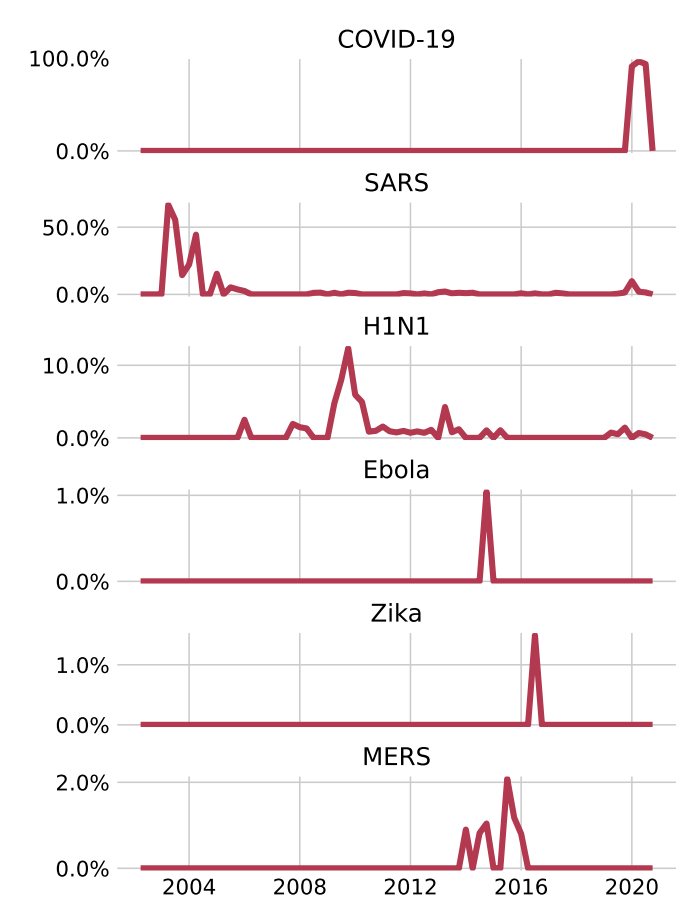

(b) United States

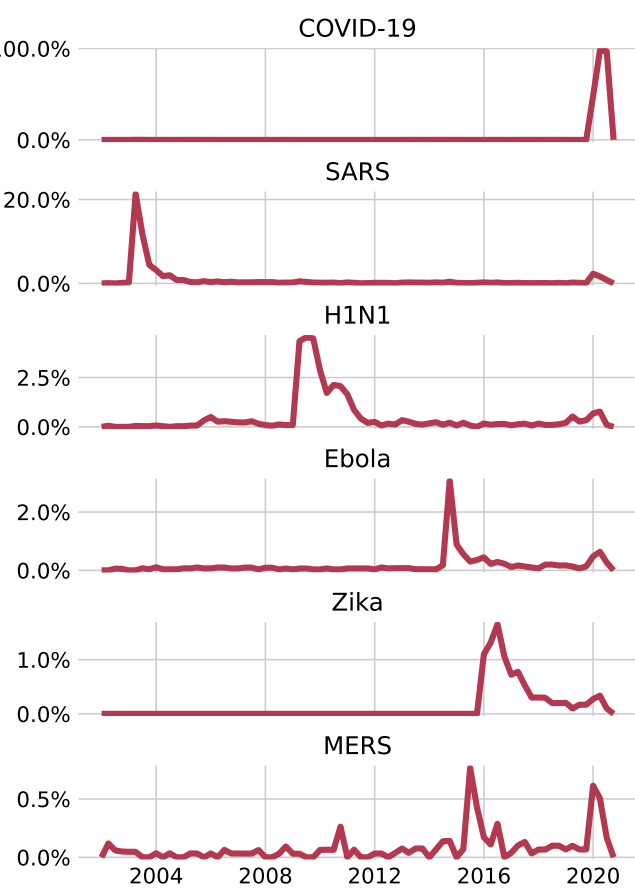

(c) Europe

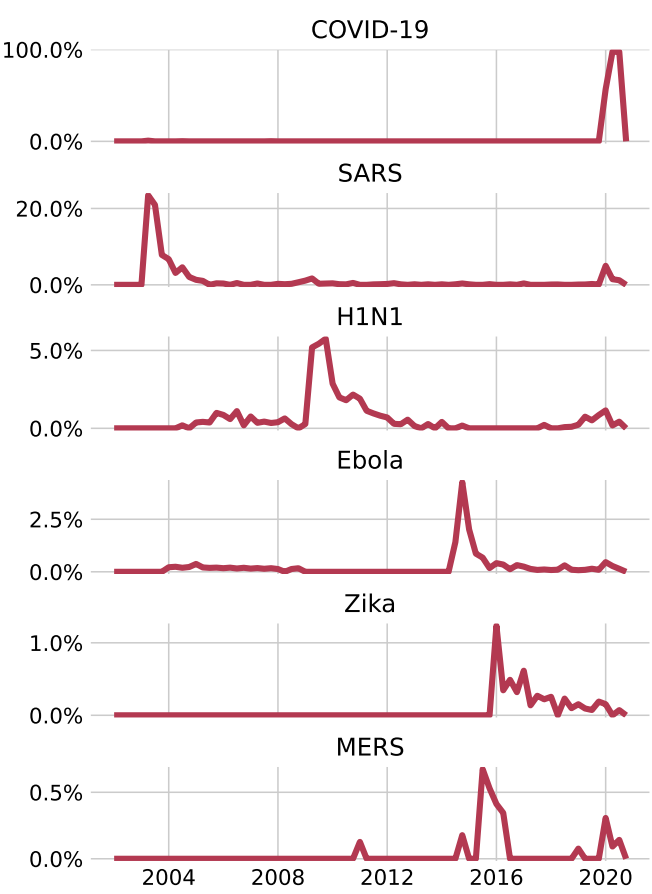

Notes: This figure plots the percentage of earnings calls discussing epidemic diseases (COVID-19, SARS, H1N1, Ebola, Zika, and MERS) by quarter from January 2002 through December 2020, separately for firms headquartered in China (Panel a), the United States (Panel b), and Europe (Panel c). 
Appendix Figure 2: Time-series of average COVID-19 sentiment $_{i, t}$, and risk $_{i, t}$ by region

(a) Regional average of COVID-19 sentiment $i, t$ over time

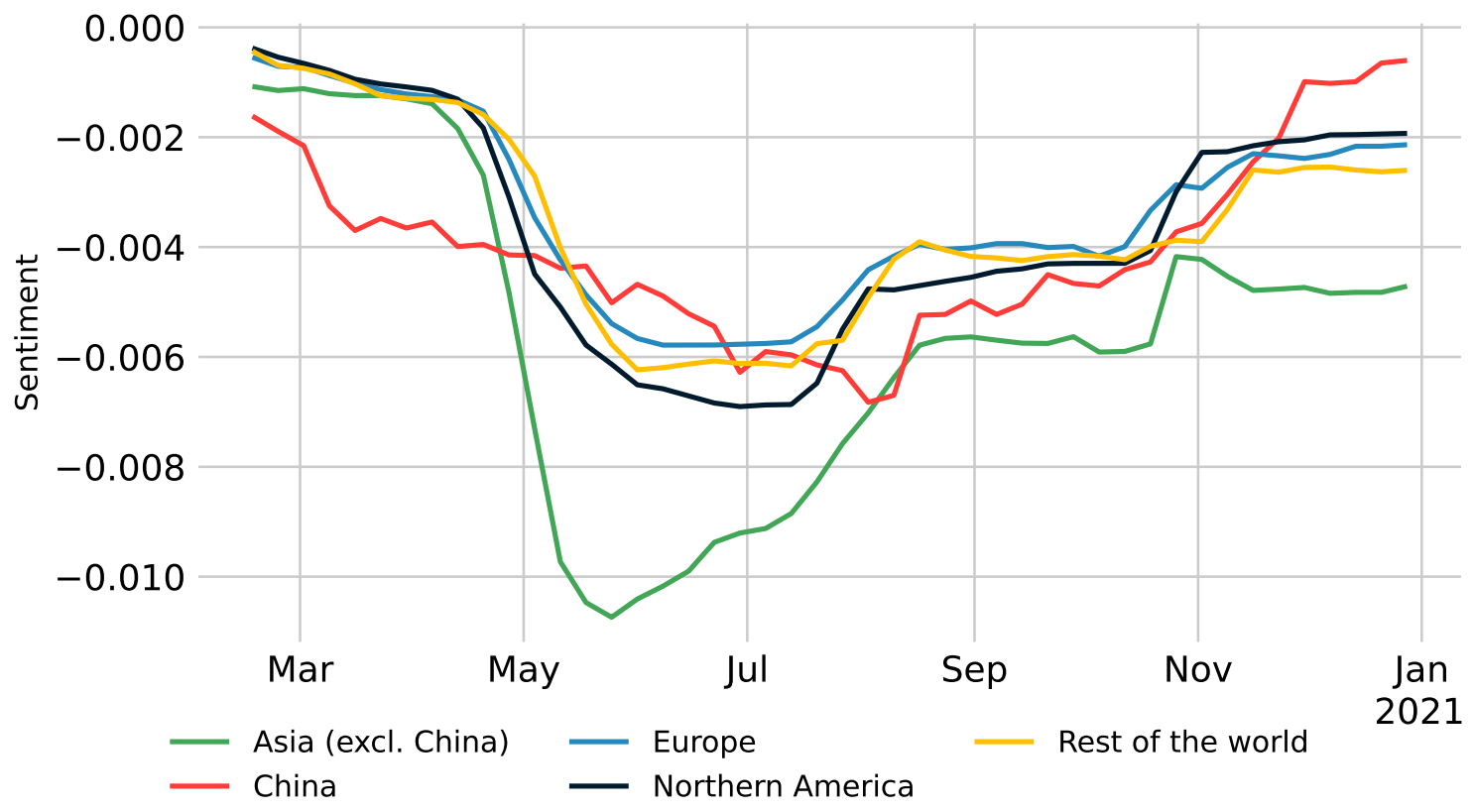

(b) Regional average of COVID-19 risk $_{i, t}$ over time

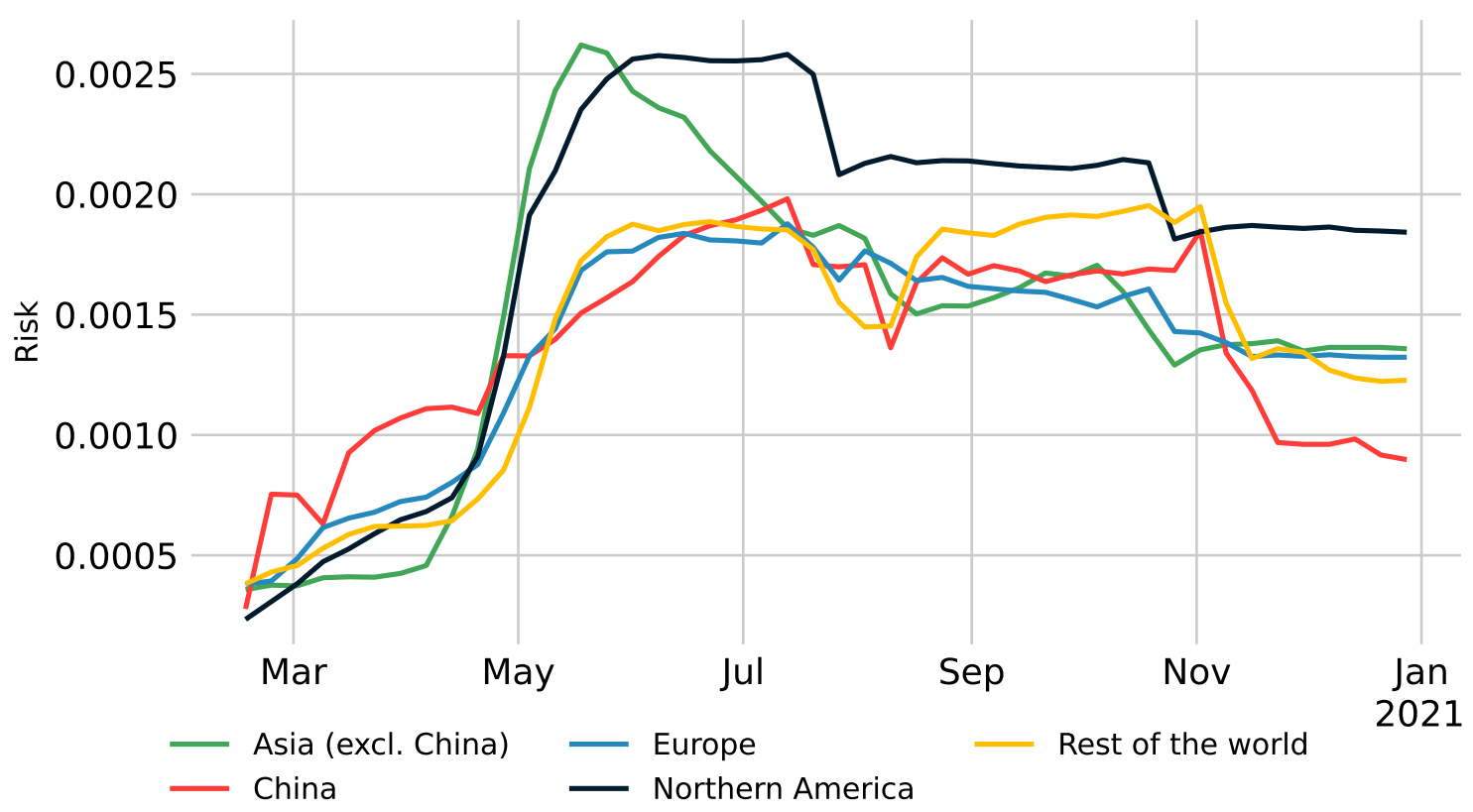

Notes: This figure plots the weekly average of COVID-19 sentiment ${ }_{i, t}$, and COVID-19 risk ${ }_{i, t}$ by region-i.e., Asia (excl. China), China, Europe, Northern America, Rest of the world - using all earnings conference calls held in the indicated time period by firms headquartered in the indicated region. The time series are smoothed using a weighted moving average over the last 12 weeks using the number of earnings conference calls as weights. 
Appendix Figure 3: Topic word clouds based on LDA
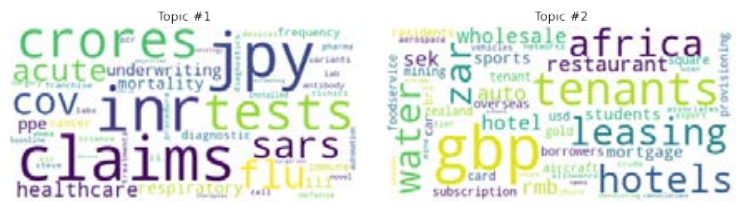

(a) Word clouds for $n=2$
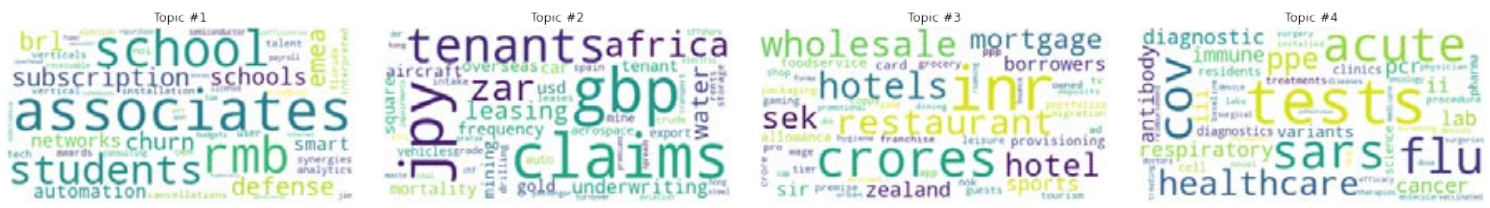

(b) Word clouds for $n=4$
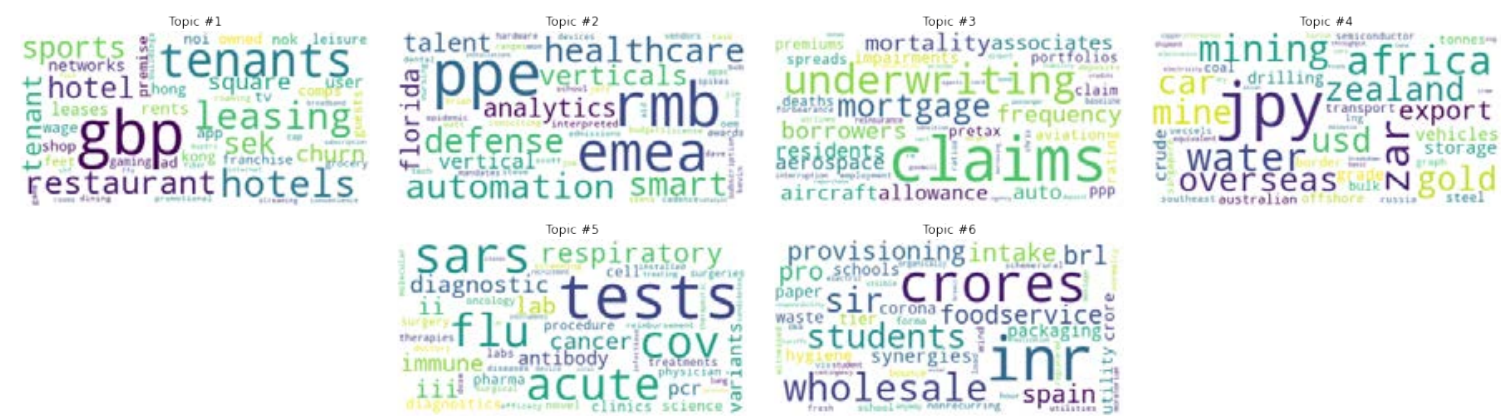

(c) Word clouds for $n=6$
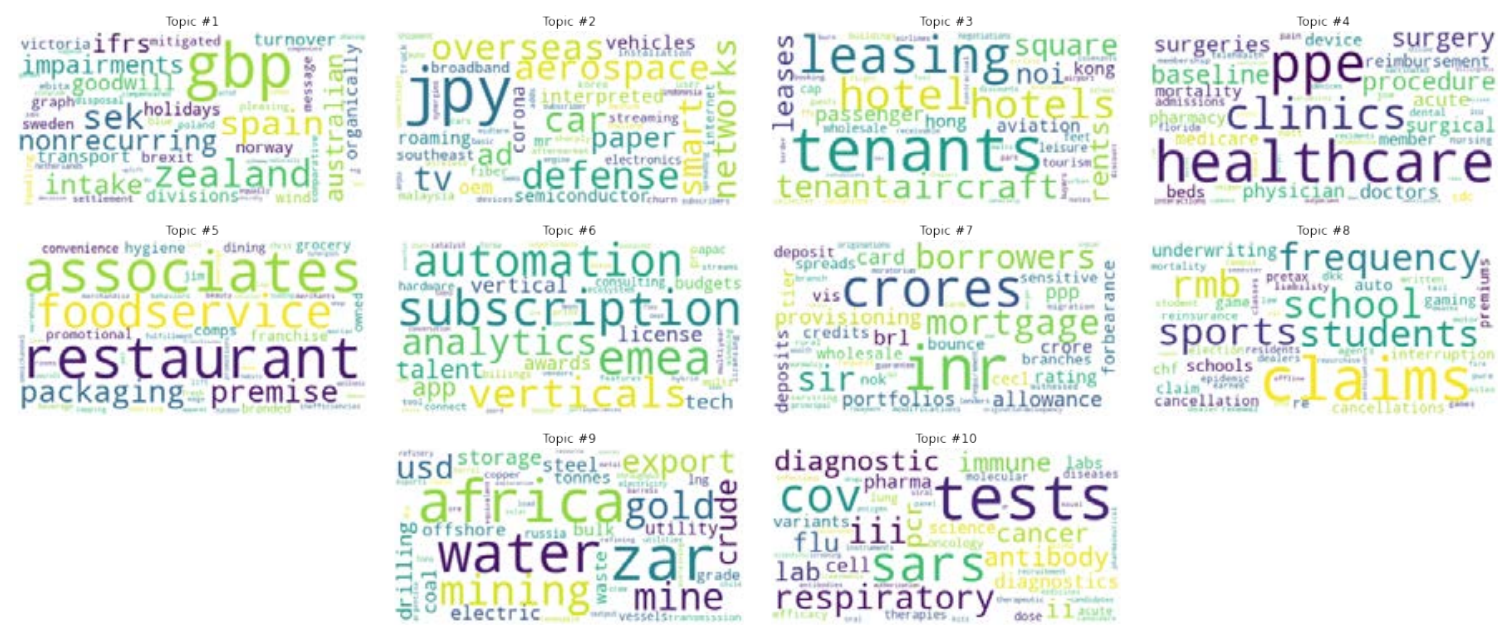

(d) Word clouds for $n=10$

\begin{abstract}
Notes: This figure shows word clouds corresponding to Latent Dirichlet Allocation (LDA) topic models with $n \in\{2,4,6,10\}$ topics. The underlying data are all sentence triples; i.e., text fragments consisting of three consecutive sentences by the same speaker, with the middle sentence containing a keyword related to COVID-19 as specified in Appendix Table 2. We split the sentence triples into words, remove stopwords, and stem the remaining tokens. We concatenate all tokens from sentence triples belonging to the same earnings call into one vector, and keep the 10,000 highest-ranking tokens when sorted on tf $\times$ idf. The unit of analysis for the LDA model consists of the remaining tokens from all sentence triples belonging to the same earnings call. We implement LDA using the collapsed Gibbs sampling algorithm (using the priors recommended by Griffiths and Steyvers (2004)) and use the Python module developed by Hansen et al. (2018) and available on https://github.com/alan-turing-institute/topic-modelling-tools.
\end{abstract}


Appendix Figure 4: Confusion matrices for disease-related topics on training data

(a) Demand impacts

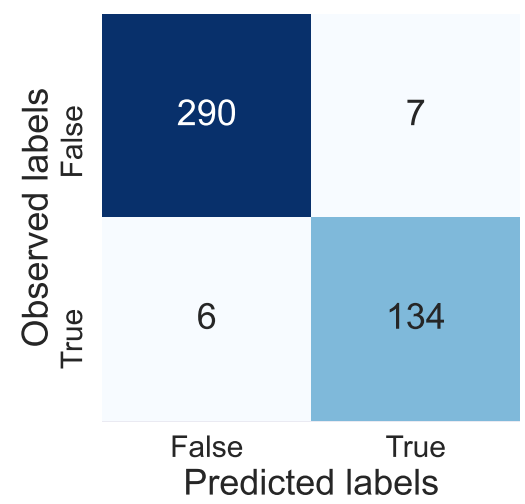

(d) Cost reductions

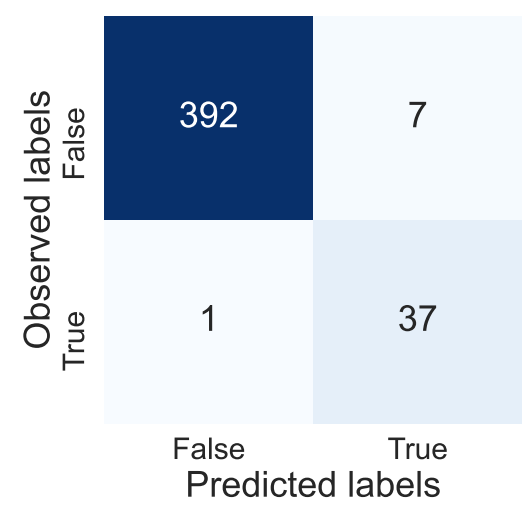

(b) Supply impacts (supply chain)

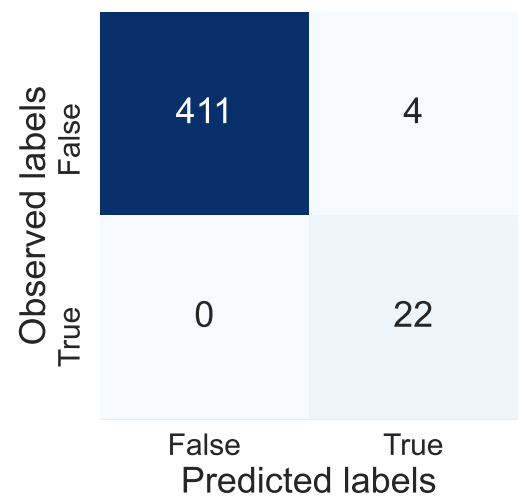

(e) Financial adjustments

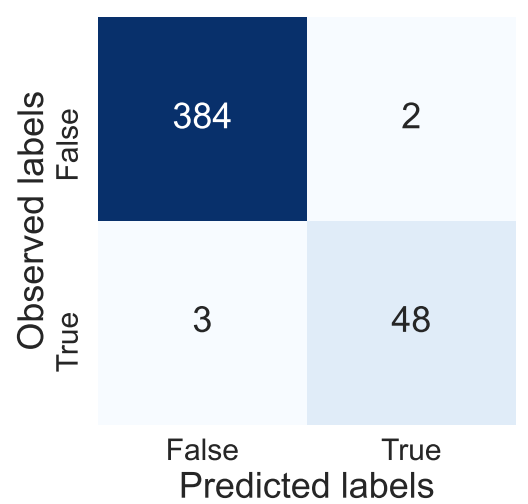

(c) Supply impacts (production and operations)

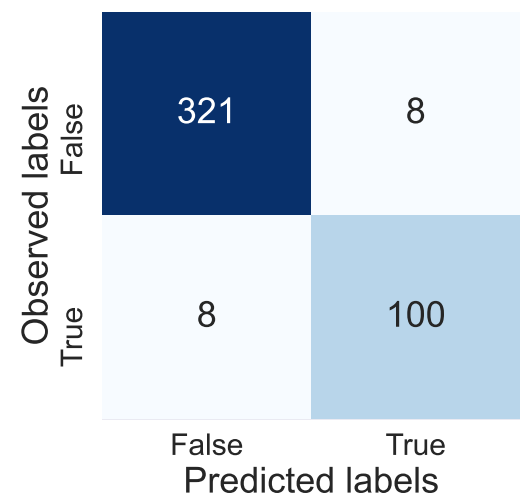

(f) Government assistance

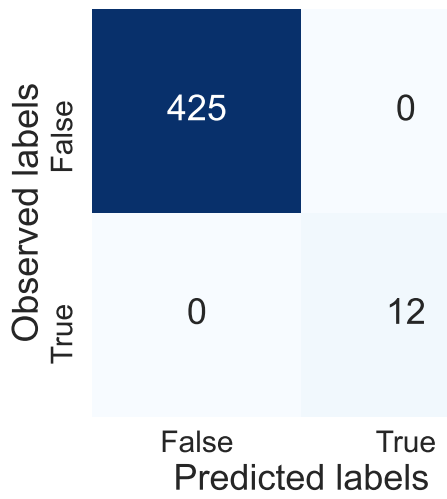

Notes: This figure shows confusion matrices, with two dimensions ("predicted" and "observed"), summarizing the performance of our pattern-based classifier for each individual topic (Panel (a) 'demand impacts,' (b) 'supply impacts (supply chain),' (c) 'supply impacts (production and operations),' (d) 'cost reductions,' (e) 'financial adjustments,' (f) 'government assistance') on the training dataset of manually-classified sentence triples about the topic, showing the number of true positives, false positives, true negatives, and false negatives. 
Appendix Figure 5: COVID-19-speech topic decomposition, including Unspecific or Other

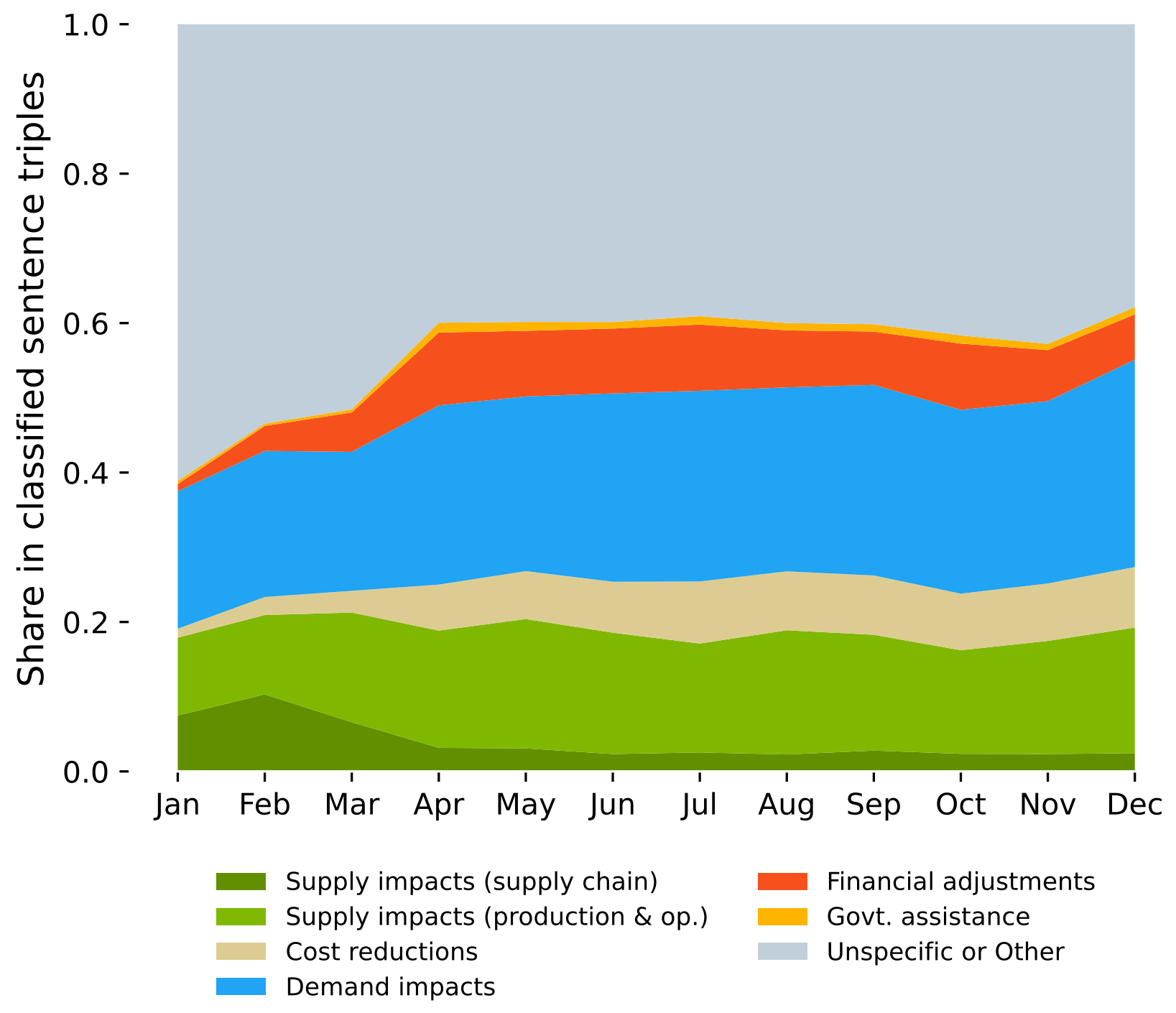

Notes: This figure is similar to Figure 5 but now also includes the share of sentence triples mentioning COVID-19 that cannot be classified to one of the following topic categories: 'supply impacts' (i.e., 'supply chain' and 'production and operations'), 'cost reductions,' 'demand impacts,' 'financial adjustments,' 'government assistance'. We label this remaining category Unspecific or Other. A sentence triple is defined as three consecutive sentences (if available) by the same speaker with the middle sentence containing a COVID-19-related keyword. Sentence triples assigned to more than one topic are duplicated for the purpose of determining the denominator; by doing so, shares add up to one. Sentence triples are obtained from transcripts of all earnings conference calls held from January through December 2020. 
Appendix Figure 6: Regional and sectoral decomposition of COVID-19-related topic shares

(a) By region
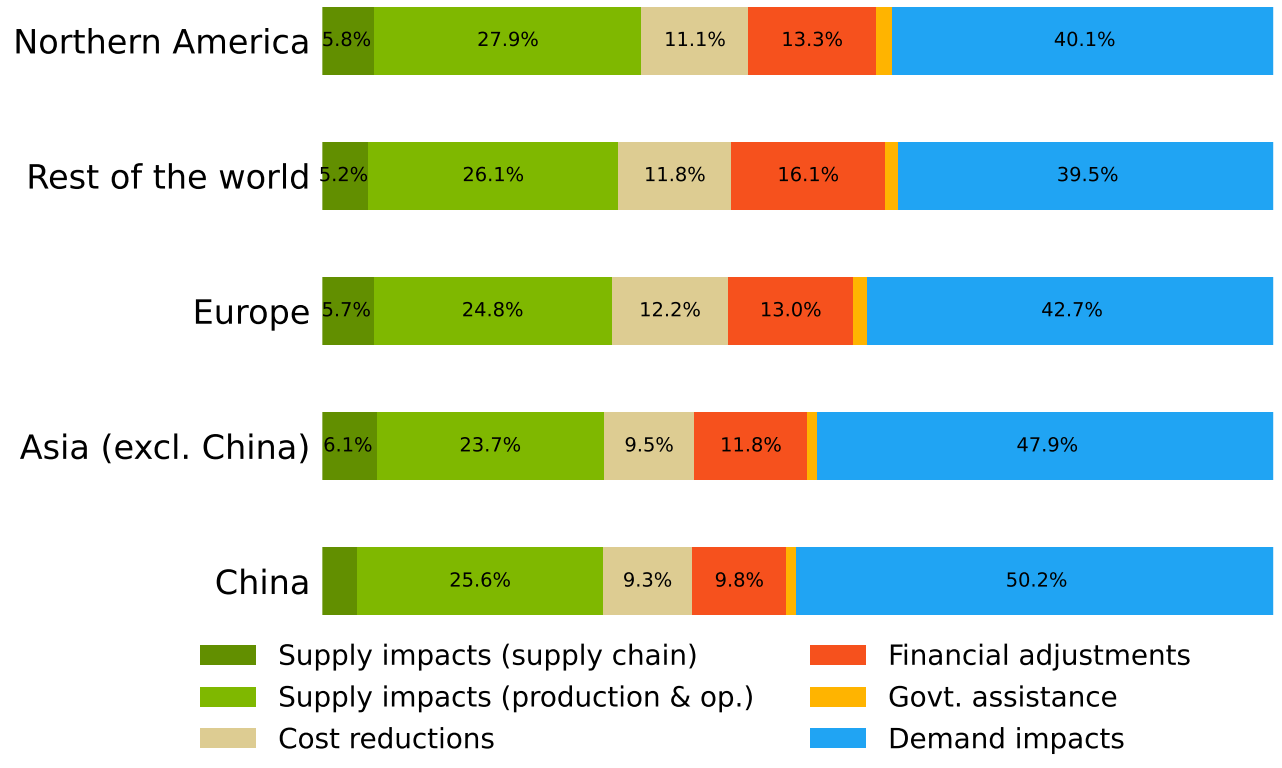

(b) By sector

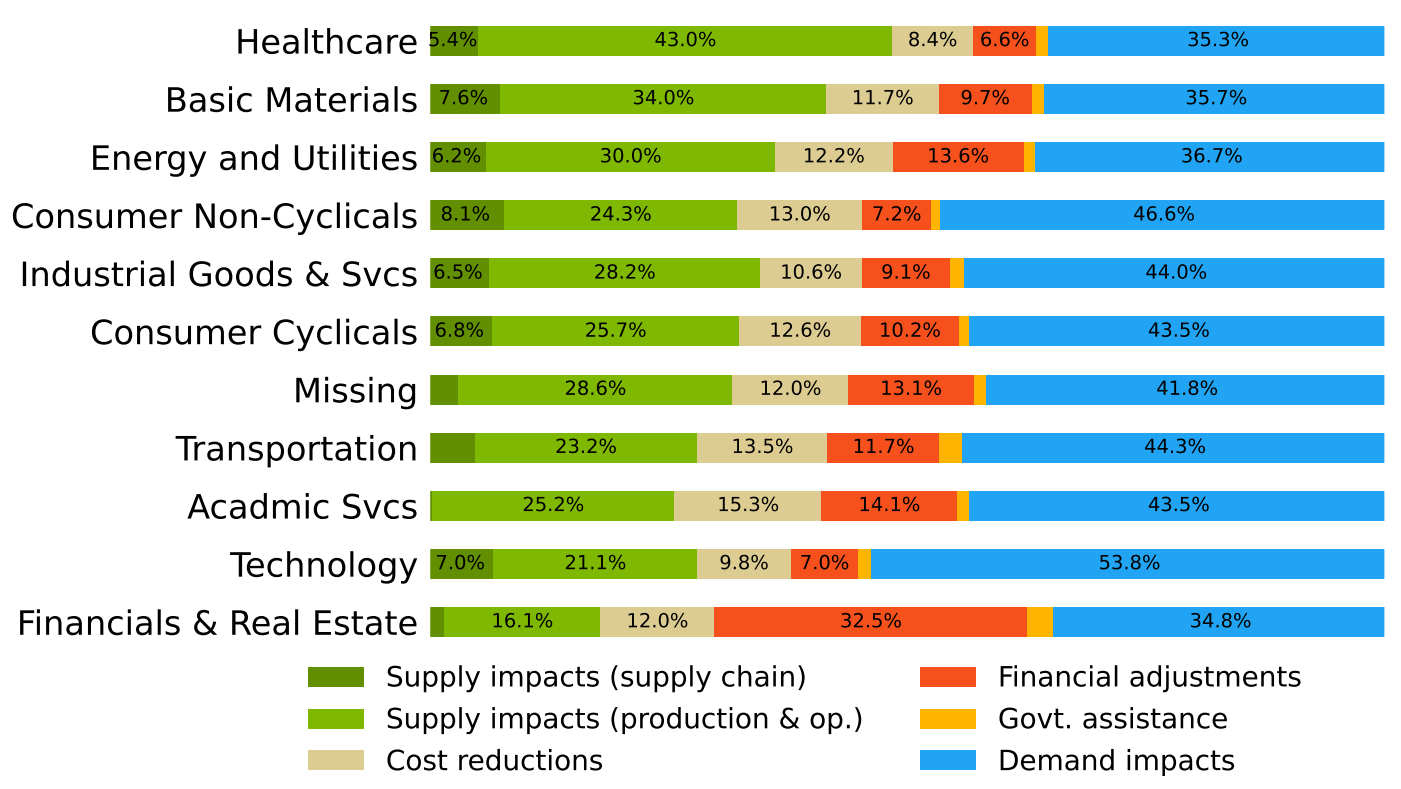

Notes: This figure plots the regional (Panel a) and sectoral (Panel b) average share of sentence triples mentioning COVID19 on the following five topics: 'supply issues' (i.e., 'supply impacts (supply chain)' and 'supply impacts (production and operations)'), 'cost reductions,' 'demand impacts,' 'financial adjustments,' 'government assistance.' A sentence triple is defined as three consecutive sentences (if available) by the same speaker with the middle sentence containing a COVID19-related keyword. Sentence triples assigned to more than one topic are duplicated for the purpose of determining the denominator; by doing so, shares add up to one. Sentence triples are obtained from transcripts of all earnings conference calls held from January through December 2020. The sector classification corresponds to the "Economic Sector" as obtained from the Refinitiv Eikon database. 


\section{Appendix Figure 7: Parallel trend assumption test}
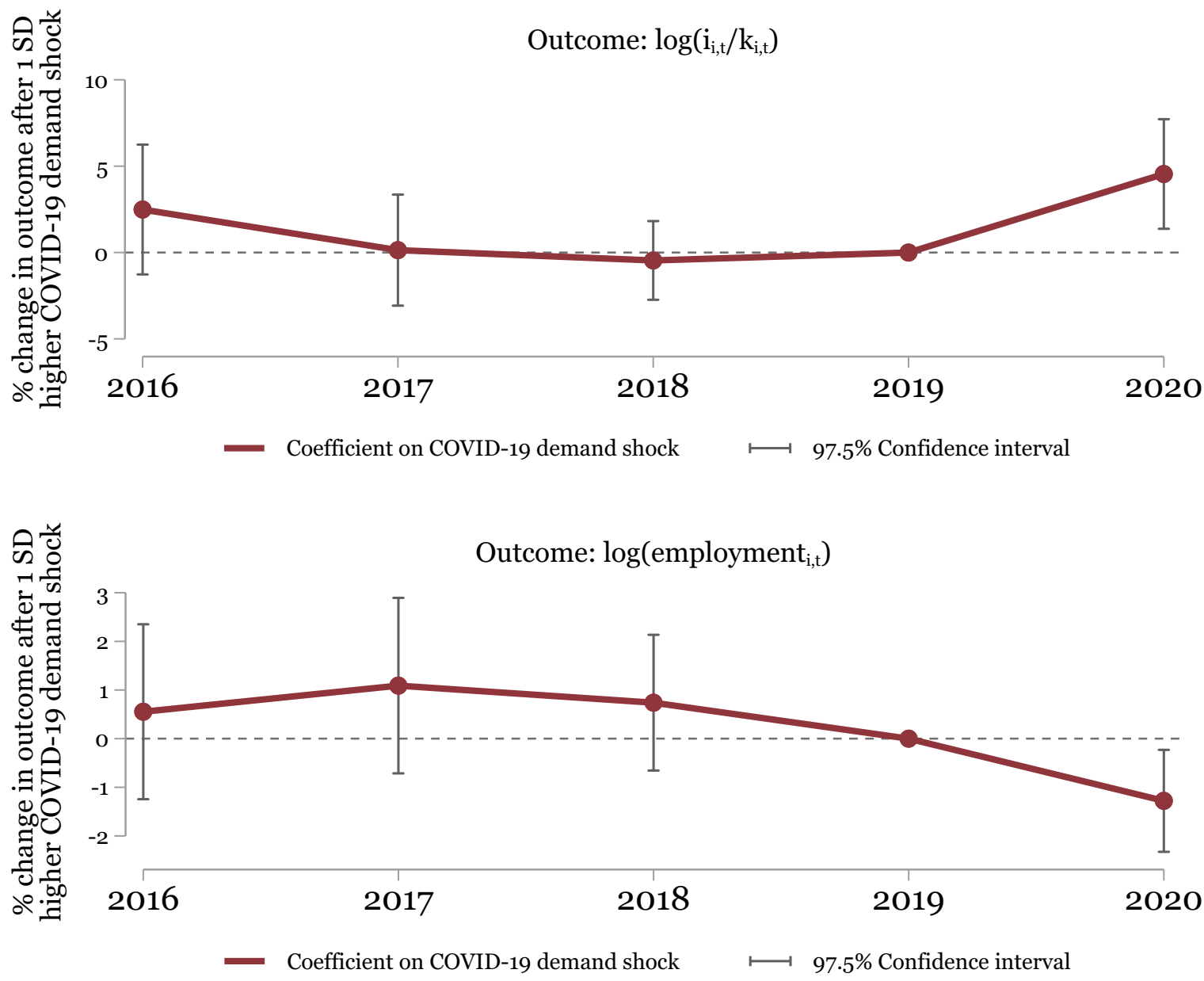

Notes: This figure plots the coefficient estimates and standard errors for $\beta_{1}^{t}$ from the following firm-year level regression:

$$
\begin{aligned}
y_{i, t}= & \delta_{s(i)}+\gamma_{t}+\sum_{t} \beta_{1}^{t} \text { Average COVID-19 net demand shock }(\text { std. })_{i, t} \times \text { Time }_{t} \\
& +\sum_{t} \beta_{2}^{t} \text { Average COVID-19 negative supply shock }(\text { std. })_{i, t} \times \text { Time }_{t}+\mathbf{x}_{i t}^{\prime} \eta+\varepsilon_{i, t}
\end{aligned}
$$

where $y_{i, t}$ is $\log \left(i_{i, t} / k_{i, t}\right)$ in the top panel and $\log \left(\right.$ employment $\left.t_{i, t}\right)$ in the bottom panel; $\delta_{i}$ and $\gamma_{t}$ are firm and quarter fixed effects, respectively; COVID-19 net demand shock and COVID-19 negative supply shock are measured as defined in Section 3; and $\mathbf{x}_{i t}$ contains the log of firm $i$ 's total assets in 2019 interacted with a post dummy variable. All other variables are as defined in Table 1. The sample of firms is restricted to large US firms (more than 500 employees) as in column 3 of Table 9 . The sample period is restricted to $t=\{2016, \ldots, 2020\}$. Standard errors are clustered by firm. ${ }^{*}, * *$, and $* * *$ indicate statistical significance at the 10,5 , and 1 percent significance, respectively. 


\section{Appendix Table 1: Distribution of sample earnings conference calls by country}

\begin{tabular}{|c|c|c|c|c|c|c|c|c|c|}
\hline Country & Freq. & Perc. & Cum. & Firms & Country & Freq. & Perc. & Cum. & Firms \\
\hline Argentina & 531 & $0.16 \%$ & $0.16 \%$ & 21 & Macao & 9 & $0.00 \%$ & $24.17 \%$ & 1 \\
\hline Australia & 3928 & $1.16 \%$ & $1.31 \%$ & 448 & Malaysia & 290 & $0.09 \%$ & $24.26 \%$ & 24 \\
\hline Austria & 938 & $0.28 \%$ & $1.59 \%$ & 35 & Malta & 45 & $0.01 \%$ & $24.27 \%$ & 6 \\
\hline Bahamas & 58 & $0.02 \%$ & $1.61 \%$ & 3 & Marshall Islands & 35 & $0.01 \%$ & $24.28 \%$ & 1 \\
\hline Bahrain & 20 & $0.01 \%$ & $1.61 \%$ & 3 & Mauritius & 13 & $0.00 \%$ & $24.29 \%$ & 3 \\
\hline Bangladesh & 3 & $0.00 \%$ & $1.61 \%$ & 1 & Mexico & 2361 & $0.70 \%$ & $24.98 \%$ & 108 \\
\hline Belgium & 1049 & $0.31 \%$ & $1.92 \%$ & 46 & Monaco & 294 & $0.09 \%$ & $25.07 \%$ & 11 \\
\hline Bermuda & 2923 & $0.86 \%$ & $2.79 \%$ & 97 & Morocco & 15 & $0.00 \%$ & $25.07 \%$ & 1 \\
\hline Brazil & 4676 & $1.38 \%$ & $4.16 \%$ & 187 & Netherlands & 2962 & $0.87 \%$ & $25.95 \%$ & 108 \\
\hline British Virgin Islands & 31 & $0.01 \%$ & $4.17 \%$ & 4 & New Zealand & 478 & $0.14 \%$ & $26.09 \%$ & 62 \\
\hline Canada & 21044 & $6.20 \%$ & $10.38 \%$ & 970 & Nigeria & 104 & $0.03 \%$ & $26.12 \%$ & 15 \\
\hline Cayman Islands & 418 & $0.12 \%$ & $10.50 \%$ & 18 & Norway & 2158 & $0.64 \%$ & $26.76 \%$ & 114 \\
\hline Channel Islands & 567 & $0.17 \%$ & $10.67 \%$ & 46 & Oman & 58 & $0.02 \%$ & $26.77 \%$ & 3 \\
\hline Chile & 833 & $0.25 \%$ & $10.91 \%$ & 47 & Pakistan & 16 & $0.00 \%$ & $26.78 \%$ & 6 \\
\hline China & 5117 & $1.51 \%$ & $12.42 \%$ & 358 & Panama & 122 & $0.04 \%$ & $26.81 \%$ & 3 \\
\hline Colombia & 338 & $0.10 \%$ & $12.52 \%$ & 16 & Papua New Guinea & 31 & $0.01 \%$ & $26.82 \%$ & 2 \\
\hline Costa Rica & 10 & $0.00 \%$ & $12.52 \%$ & 1 & Peru & 195 & $0.06 \%$ & $26.88 \%$ & 21 \\
\hline Cyprus & 304 & $0.09 \%$ & $12.61 \%$ & 21 & Philippines & 248 & $0.07 \%$ & $26.95 \%$ & 20 \\
\hline Czechia & 223 & $0.07 \%$ & $12.68 \%$ & 6 & Poland & 673 & $0.20 \%$ & $27.15 \%$ & 32 \\
\hline Denmark & 1876 & $0.55 \%$ & $13.23 \%$ & 62 & Portugal & 515 & $0.15 \%$ & $27.30 \%$ & 13 \\
\hline Egypt & 157 & $0.05 \%$ & $13.28 \%$ & 8 & Puerto Rico & 234 & $0.07 \%$ & $27.37 \%$ & 8 \\
\hline Faroe Islands & 14 & $0.00 \%$ & $13.28 \%$ & 1 & Qatar & 58 & $0.02 \%$ & $27.39 \%$ & 4 \\
\hline Finland & 2113 & $0.62 \%$ & $13.91 \%$ & 68 & Republic of Korea & 1312 & $0.39 \%$ & $27.78 \%$ & 46 \\
\hline France & 4003 & $1.18 \%$ & $15.09 \%$ & 166 & Romania & 37 & $0.01 \%$ & $27.79 \%$ & 4 \\
\hline Germany & 5844 & $1.72 \%$ & $16.81 \%$ & 232 & Russian Federation & 1229 & $0.36 \%$ & $28.15 \%$ & 54 \\
\hline Gibraltar & 62 & $0.02 \%$ & $16.83 \%$ & 2 & Saudi Arabia & 35 & $0.01 \%$ & $28.16 \%$ & 3 \\
\hline Greece & 1028 & $0.30 \%$ & $17.13 \%$ & 41 & Singapore & 1086 & $0.32 \%$ & $28.48 \%$ & 58 \\
\hline Hong Kong & 1409 & $0.42 \%$ & $17.54 \%$ & 117 & Slovenia & 3 & $0.00 \%$ & $28.48 \%$ & 1 \\
\hline Hungary & 206 & $0.06 \%$ & $17.61 \%$ & 4 & South Africa & 1462 & $0.43 \%$ & $28.91 \%$ & 101 \\
\hline Iceland & 59 & $0.02 \%$ & $17.62 \%$ & 4 & Spain & 2240 & $0.66 \%$ & $29.57 \%$ & 76 \\
\hline India & 4942 & $1.46 \%$ & $19.08 \%$ & 367 & Sweden & 4286 & $1.26 \%$ & $30.84 \%$ & 208 \\
\hline Indonesia & 319 & $0.09 \%$ & $19.17 \%$ & 18 & Switzerland & 3256 & $0.96 \%$ & $31.80 \%$ & 132 \\
\hline Ireland & 2417 & $0.71 \%$ & $19.89 \%$ & 79 & Taiwan & 1377 & $0.41 \%$ & $32.20 \%$ & 50 \\
\hline Isle of Man & 46 & $0.01 \%$ & $19.90 \%$ & 5 & Thailand & 387 & $0.11 \%$ & $32.32 \%$ & 24 \\
\hline Israel & 2776 & $0.82 \%$ & $20.72 \%$ & 118 & Turkey & 616 & $0.18 \%$ & $32.50 \%$ & 27 \\
\hline Italy & 2774 & $0.82 \%$ & $21.54 \%$ & 111 & Ukraine & 26 & $0.01 \%$ & $32.50 \%$ & 2 \\
\hline Japan & 7690 & $2.27 \%$ & $23.80 \%$ & 286 & United Arab Emirates & 261 & $0.08 \%$ & $32.58 \%$ & 24 \\
\hline Kazakhstan & 94 & $0.03 \%$ & $23.83 \%$ & 7 & United Kingdom & 10232 & $3.02 \%$ & $35.60 \%$ & 579 \\
\hline Kenya & 23 & $0.01 \%$ & $23.84 \%$ & 2 & United States & 218420 & $64.39 \%$ & $99.98 \%$ & 6911 \\
\hline Kuwait & 24 & $0.01 \%$ & $23.84 \%$ & 4 & Uruguay & 36 & $0.01 \%$ & $99.99 \%$ & 1 \\
\hline Luxembourg & 1114 & $0.33 \%$ & $24.17 \%$ & 53 & Venezuela & 19 & $0.01 \%$ & $100.00 \%$ & 2 \\
\hline
\end{tabular}

Notes: This table tabulates the distribution of sample earnings conference calls, held between January 1, 2002 and December 31, 2020, by firms' headquarters country. The column Freq. reports the number of earnings conference calls by firms from a particular country; the column Perc. indicates the percentage of all 2002-2020 earnings conference calls held by firms from that country; the column Cum. cumulatively sums those percentages; and the column Firms reports the number of sample firms headquartered in that country. 
Appendix Table 2: Disease-related keywords

\begin{tabular}{ll}
\hline SARS & MERS \\
\hline 'sars' & 'merscov' \\
'severe acute respiratory syndrome' & 'middle east respiratory syndrome' \\
& 'mers' \\
\hline Ebola & H1N1 \\
\hline 'ebola' & 'hn' \\
& 'swine flu' \\
& 'ahn' \\
\hline Zika & COVID-19 \\
\hline 'zika' & 'sarscov' \\
& 'coronavirus' \\
& 'corona virus' \\
& 'ncov' \\
& 'covid' \\
\hline
\end{tabular}

Notes: This table lists for each of the six diseases considered in the paper (i.e., SARS, MERS, Ebola, H1N1, Zika, and COVID-19), as described in Section 2.1, the list of keywords used to identify a disease. In pre-processing, we remove all non-letters and, in addition, set all text to lower case (hence, for example, "H1N1" becomes "hn" and "COVID-19" becomes "covid"). 
Appendix Table 3: Frequency risk or uncertainty synonyms in disease-related discussions

\begin{tabular}{|c|c|c|c|}
\hline Word & Frequency & Word & Frequency \\
\hline uncertainty & 4052 & bet & 9 \\
\hline risk & 1812 & queries & 9 \\
\hline uncertainties & 1386 & unforeseeable & 9 \\
\hline uncertain & 889 & risky & 8 \\
\hline risks & 816 & sticky & 7 \\
\hline unknown & 309 & reservation & 7 \\
\hline threat & 298 & halting & 7 \\
\hline exposed & 214 & suspicion & 7 \\
\hline doubt & 184 & riskier & 6 \\
\hline possibility & 153 & unsettled & 6 \\
\hline fear & 153 & dilemma & 4 \\
\hline unpredictable & 146 & apprehension & 4 \\
\hline variable & 144 & tentative & 3 \\
\hline unclear & 126 & undetermined & 3 \\
\hline chance & 76 & jeopardize & 3 \\
\hline pending & 71 & query & 3 \\
\hline varying & 70 & irregular & 2 \\
\hline variability & 59 & unsafe & 2 \\
\hline likelihood & 38 & hazardous & 2 \\
\hline prospect & 30 & hesitancy & 2 \\
\hline instability & 29 & undecided & 2 \\
\hline unpredictability & 27 & erratic & 2 \\
\hline probability & 24 & precarious & 1 \\
\hline tricky & 22 & hairy & 1 \\
\hline dangerous & 20 & gamble & 1 \\
\hline hesitant & 18 & unreliable & 1 \\
\hline doubtful & 18 & unresolved & 1 \\
\hline fluctuating & 15 & jeopardy & 1 \\
\hline speculative & 12 & faltering & 1 \\
\hline danger & 11 & fickleness & 1 \\
\hline unstable & 11 & vague & 1 \\
\hline insecurity & 10 & insecure & 1 \\
\hline hazard & 10 & hesitating & 1 \\
\hline unsure & 9 & debatable & 1 \\
\hline risking & 9 & & \\
\hline
\end{tabular}

Notes: This table shows the frequency across all transcripts of earnings conference calls held between Q1-2020 and Q4-2020 of all singleword synonyms of "risk," "risky," "uncertain," and "uncertainty" as given in the Oxford Dictionary (excluding "question" and "questions") that appear within 10 words of a disease-related keyword for each of the six diseases considered in the paper: SARS, MERS, H1N1, Zika, Ebola, and COVID-19. 


\section{Appendix Table 4: Frequently used tone words in disease-related discussions}

\begin{tabular}{|c|c|c|c|c|c|c|c|}
\hline Positive word & Frequency & Positive word & Frequency & Negative word & Frequency & Negative word & Frequency \\
\hline despite & 4310 & gains & 151 & crisis & 6995 & stress & 291 \\
\hline strong & 3416 & highest & 149 & challenges & 3716 & suspended & 284 \\
\hline good & 2644 & enhanced & 148 & negative & 2548 & restructuring & 284 \\
\hline positive & 1972 & positively & 144 & decline & 1904 & slower & 270 \\
\hline able & 1920 & enabled & 134 & disruption & 1821 & weakness & 269 \\
\hline better & 1280 & incredibly & 129 & against & 1662 & recession & 261 \\
\hline great & 1231 & progressing & 127 & difficult & 1561 & closure & 247 \\
\hline opportunities & 1102 & easy & 124 & challenging & 1385 & challenged & 229 \\
\hline progress & 1058 & enable & 124 & disruptions & 1087 & cancellations & 223 \\
\hline opportunity & 963 & strengthen & 122 & negatively & 1020 & postponed & 221 \\
\hline pleased & 727 & profitable & 118 & loss & 1005 & difficulty & 216 \\
\hline benefit & 726 & perfect & 116 & delays & 994 & slowing & 216 \\
\hline best & 671 & efficiencies & 110 & delayed & 945 & serious & 215 \\
\hline improved & 574 & greatly & 110 & declined & 829 & exposed & 214 \\
\hline improvement & 560 & progressed & 109 & losses & 789 & forced & 208 \\
\hline confident & 557 & attractive & 108 & late & 762 & recall & 206 \\
\hline strength & 539 & incredible & 108 & concerns & 761 & lack & 205 \\
\hline stronger & 512 & impressive & 106 & slowdown & 730 & weaker & 203 \\
\hline greater & 477 & stability & 104 & challenge & 693 & unexpected & 194 \\
\hline improve & 451 & benefiting & 101 & closed & 676 & problems & 194 \\
\hline profitability & 448 & efficient & 96 & claims & 637 & prevention & 193 \\
\hline leading & 390 & enhance & 96 & severe & 613 & suffered & 190 \\
\hline stable & 368 & stabilize & 94 & shutdown & 605 & exacerbated & 185 \\
\hline effective & 364 & stabilized & 90 & volatility & 561 & canceled & 184 \\
\hline successfully & 329 & strengthened & 87 & delay & 556 & doubt & 184 \\
\hline achieved & 322 & innovative & 85 & closures & 543 & strains & 181 \\
\hline optimistic & 296 & boost & 83 & critical & 540 & dropped & 180 \\
\hline successful & 285 & greatest & 82 & unfortunately & 522 & unfavorable & 180 \\
\hline happy & 262 & exciting & 81 & adverse & 504 & deterioration & 178 \\
\hline benefited & 259 & achieving & 80 & slowed & 487 & interruption & 176 \\
\hline success & 259 & gained & 77 & shutdowns & 481 & worst & 173 \\
\hline favorable & 251 & win & 76 & lost & 447 & stopped & 173 \\
\hline improving & 246 & strengthening & 76 & slow & 427 & worse & 171 \\
\hline advantage & 244 & advancing & 75 & concern & 416 & difficulties & 171 \\
\hline proactive & 236 & strongest & 67 & declines & 416 & suspension & 170 \\
\hline proactively & 231 & efficiently & 66 & bad & 388 & suffering & 168 \\
\hline achieve & 230 & easier & 64 & shut & 387 & unemployment & 166 \\
\hline improvements & 220 & achievement & 64 & force & 380 & volatile & 162 \\
\hline tremendous & 218 & improves & 63 & downturn & 365 & overcome & 162 \\
\hline rebound & 198 & diligently & 62 & concerned & 362 & prolonged & 158 \\
\hline encouraged & 198 & enabling & 62 & severely & 357 & declining & 155 \\
\hline exceptional & 195 & exceptionally & 62 & problem & 322 & fear & 153 \\
\hline efficiency & 192 & gaining & 59 & severity & 306 & unable & 147 \\
\hline excellent & 185 & valuable & 57 & adversely & 305 & unpredictable & 146 \\
\hline encouraging & 180 & advantages & 56 & closing & 304 & caution & 144 \\
\hline excited & 180 & resolve & 52 & impairment & 304 & impairments & 138 \\
\hline leadership & 178 & beneficial & 51 & disrupted & 301 & destruction & 131 \\
\hline gain & 158 & fantastic & 47 & strain & 300 & complications & 129 \\
\hline innovation & 155 & rebounded & 47 & threat & 298 & fallout & 128 \\
\hline collaboration & 153 & outperformed & 46 & weak & 292 & cut & 125 \\
\hline
\end{tabular}

Notes: This table shows the frequency across all transcripts of earnings conference calls held between Q1-2020 and Q4-2020 of the top 100 positive and negative tone words from Loughran and McDonald (2011) (note: their list contains 354 positive and 2,352 negative tone words) that appear within 10 words of a disease-related keyword for each of the six diseases considered in the paper: SARS, MERS, H1N1, Zika,

Ebola, and COVID-19. 
Appendix Table 5: Does epidemic data predict firm-level COVID-19 measures?

\begin{tabular}{|c|c|c|c|c|}
\hline & \multicolumn{2}{|c|}{ COVID-19 negative sentiment $t_{i, t}$} & \multicolumn{2}{|c|}{ COVID-19 exposure $_{i, t}$} \\
\hline & $(1)$ & $(2)$ & (3) & (4) \\
\hline New cases per $100,000_{C(i), t}$ & $\begin{array}{c}0.006^{* * *} \\
(0.001)\end{array}$ & & $\begin{array}{c}0.105^{* * *} \\
(0.003)\end{array}$ & \\
\hline New deaths per $100,000_{C(i), t}$ & & $\begin{array}{c}0.224^{* * *} \\
(0.049)\end{array}$ & & $\begin{array}{c}4.237^{* * *} \\
(0.112)\end{array}$ \\
\hline COVID-19 exposure $_{i, t}$ & $\begin{array}{c}0.411^{* * *} \\
(0.007)\end{array}$ & $\begin{array}{c}0.410^{* * *} \\
(0.007)\end{array}$ & & \\
\hline$R^{2}$ & 0.614 & 0.614 & 0.064 & 0.088 \\
\hline$N$ & 16,563 & 16,563 & 16,563 & 16,563 \\
\hline
\end{tabular}

Notes: This table reports estimated coefficients and standard errors from firm-quarter level regressions for Q1-2020Q1 through Q3-2020. New cases per 100,000 ${ }_{C(i), t}$ is the number of confirmed COVID-19 cases per 100,000 in quarter $t$ of firm $i$ 's headquarters country $C$; similarly, New deaths per 100,000 $C(i), t$ is defined for the number of deceased COVID-19 patients per 100,000. Data for both variables are obtained from Google's COVID-19 Open Data: https://console.cloud.google.com/marketplace/product/ bigquery-public-datasets/covid19-open-data. Country-quarter cells with less than 25 firms are excluded. All regressions control for the $\log$ of firm assets. Standard errors are robust. ${ }^{* * *},{ }^{* *}$, and ${ }^{*}$ denote statistical significance at the 1,5 , and $10 \%$ level, respectively. 
Appendix Table 6: Timing of COVID-19-sentiment and -risk discussions

PANEL A: COVID-19 sentiment

\section{Ongoing or ex-post in nature: $\quad 81 \%$ Future or ex-ante in nature:}

Example: "Yes, there's been no change in Europe with Roche. Other than just the COVID impact on their clinic access with patients and providers. We are seeing that rebound as well." (Senseonics Holdings Inc; June 9, 2020)
Example: "We might also continue to experience production constraints, and we're entering Q3 and the high season at a lower inventory level than normal as our production rate in Q2 was impacted by the pandemic." (Electrolux AB; July 17, 2020)

PANEL B: COVID-19 risk

\section{Ongoing or ex-post in nature:}

Example: "We recognized early the potential severity of the worldwide COVID-19 pandemic, and we moved quickly to adjust our operating budget to reflect that uncertainty, including a voluntary $10 \%$ salary cut for our executive team; a freeze on raises during 2020; and a cut in our regulatory budget, among other serious cuts." (Marrone Bio Innovations Inc; May 11, 2020)

Notes: This table shows the results from a human audit on the timing (i.e., ongoing or ex-post vis-a-vis future or ex-ante in nature) of COVID-19 sentiment and risk discussions, in Panel A and B, respectively, based on a randomly-drawn sample of 100 sentence triples. A sentence triple is defined as three consecutive sentences (if available) by the same speaker with the middle sentence containing a COVID-19-related keyword. For each, in the first row, we report in the left (right) column the tabulated proportion of sentence triples-out of 100-that are ongoing or ex-post (future or ex-ante) in nature, as well as an example excerpt from a sentence triple. 
Appendix Table 7: Correlation of COVID-19 measures with realized volatility

\begin{tabular}{|c|c|c|c|c|}
\hline \multirow[t]{2}{*}{ PANEL A } & \multicolumn{4}{|c|}{ Realized volatility $_{i, t}$} \\
\hline & (1) & $(2)$ & (3) & (4) \\
\hline COVID-19 exposure $_{i, t}($ std.) & $\begin{array}{c}1.179^{* * *} \\
(0.205)\end{array}$ & & & \\
\hline COVID-19 sentiment $t_{i, t}$ (std.) & & $\begin{array}{c}-0.656^{* * *} \\
(0.187)\end{array}$ & & $\begin{array}{c}-0.602^{* * *} \\
(0.190)\end{array}$ \\
\hline COVID-19 risk i,t $_{\text {(std.) }}$ & & & $\begin{array}{c}0.521^{* * *} \\
(0.165)\end{array}$ & $\begin{array}{c}0.449^{* * *} \\
(0.168)\end{array}$ \\
\hline$R^{2}$ & 0.338 & 0.337 & 0.336 & 0.337 \\
\hline$N$ & 18,506 & 18,506 & 18,506 & 18,506 \\
\hline \multirow[t]{2}{*}{ PANEL B } & \multicolumn{4}{|c|}{ Realized volatility $y_{i, t}$} \\
\hline & $(1)$ & $(2)$ & $(3)$ & $(4)$ \\
\hline COVID-19 net demand shock ${ }_{i, t}$ (std.) & $\begin{array}{c}-0.527^{* * *} \\
(0.189)\end{array}$ & & $\begin{array}{c}-0.392^{* *} \\
(0.196)\end{array}$ & $\begin{array}{c}-0.381^{*} \\
(0.197)\end{array}$ \\
\hline COVID-19 negative supply shock ${ }_{i, t}$ (std.) & & $\begin{array}{c}0.656^{* * *} \\
(0.178)\end{array}$ & $\begin{array}{c}0.560^{* * *} \\
(0.184)\end{array}$ & $\begin{array}{c}0.486^{* * *} \\
(0.184)\end{array}$ \\
\hline COVID-19 risk ${ }_{i, t}$ (std.) & & & & $\begin{array}{c}0.390^{* *} \\
(0.169)\end{array}$ \\
\hline$R^{2}$ & 0.336 & 0.337 & 0.337 & 0.337 \\
\hline$N$ & 18,506 & 18,506 & 18,506 & 18,506 \\
\hline Quarter FE & yes & yes & yes & yes \\
\hline Sector FE & yes & yes & yes & yes \\
\hline
\end{tabular}

Notes: This table reports regression estimates at the firm-quarter level. Realized volatility is the standard deviation of firm $i$ 's daily stock return, measured during the quarter, adjusted for dividends and stock splits. COVID-19 net demand shock and COVID-19 negative supply shock are measured as defined in Section 3. All regressions control for the log of firm i's total assets in 2019 and its market beta in 2018. Sector fixed effects are defined using Refinitiv Eikon's Business sector, which has 30 sectors in our sample. Standard errors are clustered at the firm level. ${ }^{* * *},{ }^{* *}$, and ${ }^{*}$ denote statistical significance at the 1, 5, and $10 \%$ level, respectively. 
Appendix Table 8: False positive rate in final pattern matching iteration

\begin{tabular}{lc}
\hline Topic & No. false positives \\
\hline Demand impacts & $6 / 30$ \\
Supply impacts (supply chain) & $3 / 30$ \\
Supply impacts (production and operations) & $8 / 30$ \\
Cost reductions & $5 / 30$ \\
Financial adjustments & $3 / 30$ \\
Government assistance & $1 / 30$ \\
\hline
\end{tabular}

Notes: This table reports the false positive rate obtained in the final iteration of our pattern matching. Specifically, for each individual topic (i.e., 'demand impacts,' 'supply chain,' 'production and operations,' 'cost reductions,' 'financial adjustments,' and 'government assistance') we randomly drew 30 sentence triples and compare the prediction of the topic-specific pattern with a manual assessment of the triple's topic. Each row lists the number of false positives out of these thirty randomly-drawn sentence triples. A sentence triple is defined as three consecutive sentences (if available) by the same speaker with the middle sentence containing a COVID-19-related keyword. 
Appendix Table 9: Additional channel-specific restrictions on word patterns

\begin{tabular}{|c|c|}
\hline Channel: & Additional restrictions: \\
\hline $\begin{array}{l}\text { Supply impacts (supply } \\
\text { chain) }\end{array}$ & Words not allowed to be between word combinations: "million" \\
\hline \multirow[t]{2}{*}{$\begin{array}{l}\text { Supply impacts } \\
\text { (production and } \\
\text { operations) }\end{array}$} & $\begin{array}{l}\text { Words not allowed to between word combinations: "loss," } \\
\text { "fund," "demand," "revenue," "expenditure," "interest rate," } \\
\text { "customer[s]," "thank," "consumer," "sale," "payment," "cost," } \\
\text { "highlight," "result," "global economy" }\end{array}$ \\
\hline & $\begin{array}{l}\text { Word-specific restrictions: "permit" may not be preceded by } \\
\text { "condition[s]," "site" may not be followed by "deposit" or } \\
\text { "lease," and "facillity" may not be preceded by "credit" }\end{array}$ \\
\hline \multirow[t]{2}{*}{ Demand impacts } & $\begin{array}{l}\text { Words not allowed to be between word combinations: "safe," } \\
\text { "support," "testing," "help," "inventory," "liabilities," } \\
\text { "accounts payable," "loss," "expense," "result," "guidance," } \\
\text { operational," "material," "cost," "service," "payout" }\end{array}$ \\
\hline & $\begin{array}{l}\text { Word-specific restrictions: "customer," "consumer," and } \\
\text { "client" may not be preceded by "support" }\end{array}$ \\
\hline Cost reductions & $\begin{array}{l}\text { Words not allowed to be between word combinations: "safe," } \\
\text { "support," "help," "inventory," "shipment," "customer," "last } \\
\text { quarter," "last year," "guidance," "operational," "material," } \\
\text { "out-of-pocket" }\end{array}$ \\
\hline \multirow[t]{2}{*}{ Financial adjustments } & $\begin{array}{l}\text { Words not allowed to be between word combinations: "safe," } \\
\text { "support," "help," "inventory," "shipment," "customer," "last } \\
\text { quarter," "last year," "guidance," "operational," "material," } \\
\text { "out-of-pocket," "companies," "cost," "spending" }\end{array}$ \\
\hline & $\begin{array}{l}\text { Word-specific restrictions: "debt" may not be preceded by } \\
\text { "sovereign" and "cash" may not be followed by "purchase" }\end{array}$ \\
\hline \multirow[t]{2}{*}{ Government assistance } & $\begin{array}{l}\text { Words not allowed to be between word combinations: } \\
\text { "mandate," "order," "shutdown," "guideline" }\end{array}$ \\
\hline & $\begin{array}{l}\text { Word-specific restrictions: "government" may not be followed } \\
\text { by either of "affairs," "shutdown," "mandate," "order," and } \\
\text { "state" may not be followed by "affair" }\end{array}$ \\
\hline
\end{tabular}

Notes: This table lists the additional channel-specific restrictions on word patterns. 
Appendix Table 10: Example of predicted COVID-19-related sentence triple by channel

Channel Example of predicted sentence triple

Supply impacts (supply chain)

Supply impacts (production and operations)

Demand impacts

Cost reductions

Financial adjustments

Government assistance
"We have the trade tariffs, as you know, that have already led to some shifts in the global supply chains. And on top of that, I would say that now the coronavirus also has led to some additional shifts and rearrangement of global supply chains. It is not a large extent, but I would guess that some of the developments in Europe as well in North America also are the result of people trying to desperately shift supply chains so that might lead to a little bit of a compensation of the slowdown in China by Europe and the United States." (Covestro AG, 19-Feb-2020)

"Moreover, most traditional and convenience stores are closed or suffering from a significant in-store traffic decline, notably in developing countries. Overall, we estimate the impact of the COVID-19 on our group first quarter net sales growth to be between minus 2 and minus 3 points. From a global supply chain perspective, several of our factories and warehouses are closed to comply with local government regulations and guidelines." (Note: also classified as 'supply chain', 'demand') (Societe BIC SA, 23-Apr-2020)

"Revenue for the 3 months ended March 31, 2020 was \$63.5 million, an increase of $31 \%$ year-over-year and $8 \%$ sequentially. Management has determined that revenue was negatively impacted in the quarter by the COVID-19 crisis on 2 fronts: first, the company booked additional reserves due to expectations of lost patient insurance and co-pay payments lower than historical averages. And secondly, the company has estimated that lower registrations and unit intake in the latter half of March had a material impact on Q1 revenues." (iRhythm Technologies Inc, 07-May-2020)

"In response to the pandemic and in recognition of mild weather entering the year, we are executing on a series of cost-saving initiatives totaling approximately $\$ 350$ million to $\$ 450$ million or $\$ 0.35$ to $\$ 0.45$ per share. We are also keeping our regulators informed about the specific costs we are incurring related to COVID-19. First and foremost, our thoughts are with those who have been personally affected." (Duke Energy Corp, 12-May-2020)

"The ratio of allowance for credit losses to NPLs held in portfolio stood $120 \%$ compared to $91 \%$ in the previous quarter. The provision for credit losses increased by $\$ 142$ million from the prior quarter, mainly driven by the COVID-19 impact on the macroeconomic scenarios. The provision to net charge-off ratio was 302\% in the first quarter of 2020." (Popular Inc, 30-Apr-2020)

"On another note, as you will see in today's press release, we've returned the $\$ 2.8$ million PPP loan, which we had qualified for. When we first considered the loans, we carefully reviewed our financial condition and the economic impact and uncertainty caused by the coronavirus pandemic. At that time, we determined the funds were necessary to maintain our ongoing operations in accordance with the terms and conditions of CARES Act." (Note: also classified as "production and operations,' 'finance') (inTest Corp, 08-May-2020)

Notes: This table reports one predicted COVID-19-related sentence triple for each of the five channels: 'supply impacts' (i.e., 'supply chain' and 'production and operations'), 'cost reductions,' 'demand impacts,' 'financial adjustments,' and 'government assistance.' The channel label of a sentence triple is predicted using our patternbased classifier as specified in the paper. Bold text indicates the pattern match that resulted in the prediction of the channel label. If a sentence triple has multiple predicted channel labels, we do not boldface the pattern match of those other channel labels. A sentence triple is defined as three consecutive sentences (if available) by the same speaker with the middle sentence containing a COVID-19-related keyword. Sentence triples are obtained from earnings-call transcripts held from January through December 2020. 
Appendix Table 11: Hyperparameter space for grid search

\begin{tabular}{lll}
\hline Naive Bayes & Laplace smoothing parameter: & {$[0.0001,0.001,0.01,0.1,1]$} \\
\hline Logistic Regression & L2 Regularization strength: & {$[0.0001,0.001,0.01,0.1,1]$} \\
\hline Hidden layer sizes: & {$[(64),,(128,64),,(256,128,64)]$,} \\
Activation function: & {$[$ relu, tanh $]$} \\
Solver: & {$[$ adam $]$} \\
L2 penalty: & {$[0.0001,0.001,0.01]$} \\
Leedforward Neural Network & Maximum iterations: $:$ & {$[30.001,0.0001]$} \\
\hline
\end{tabular}

Notes: This table reports the parameters that we consider for the grid search of the three alternative classifiers: Naive Bayes, Logistic Regression, and Feedforward Neural Network. We use scikit-learn for both the classification algorithms and the grid search; for more information about each parameter's meaning, please consult the documentation of scikitlearn and the references therein (https://scikit-learn.org/stable/index.html). 